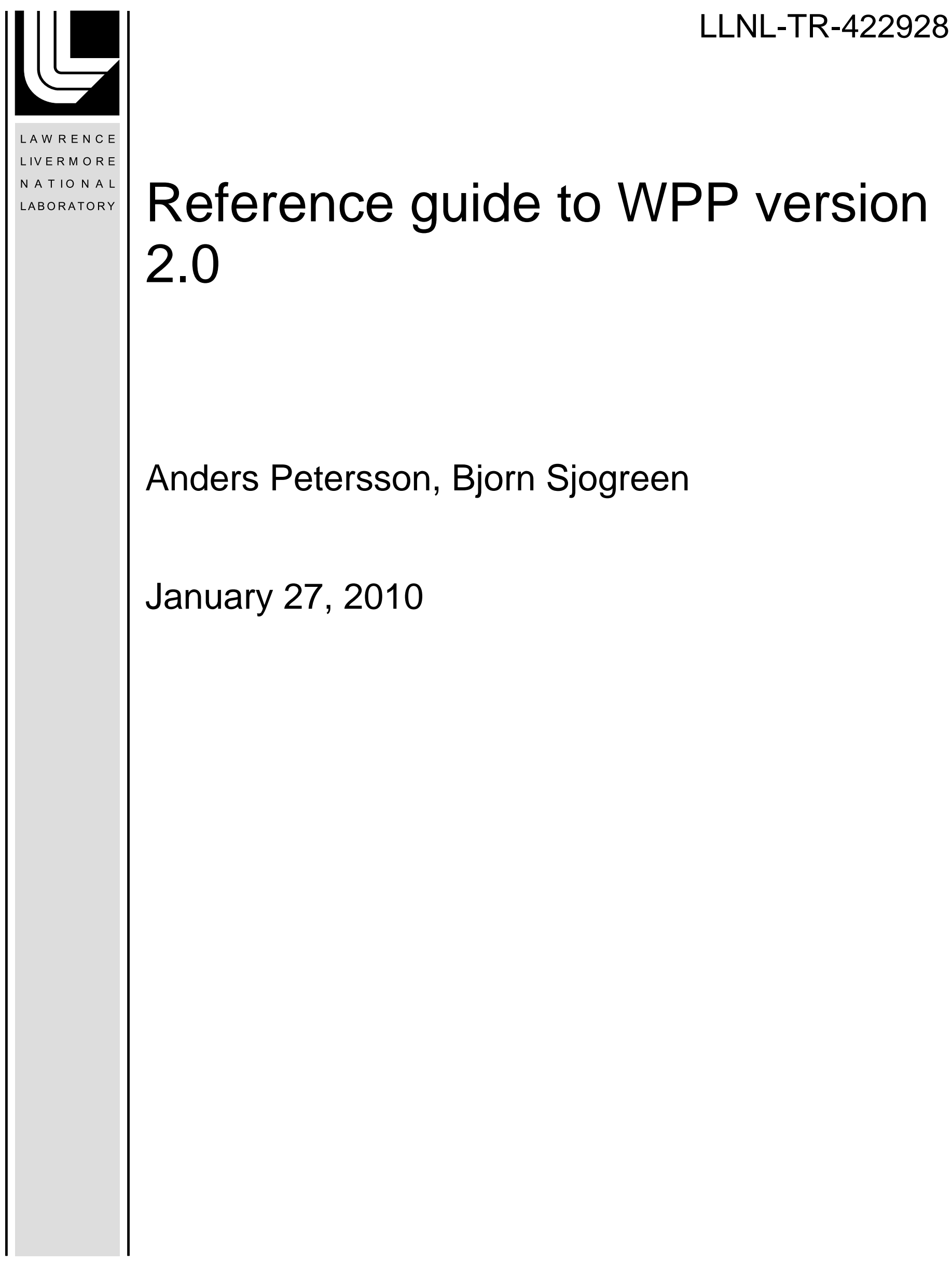


This document was prepared as an account of work sponsored by an agency of the United States government. Neither the United States government nor Lawrence Livermore National Security, LLC, nor any of their employees makes any warranty, expressed or implied, or assumes any legal liability or responsibility for the accuracy, completeness, or usefulness of any information, apparatus, product, or process disclosed, or represents that its use would not infringe privately owned rights. Reference herein to any specific commercial product, process, or service by trade name, trademark, manufacturer, or otherwise does not necessarily constitute or imply its endorsement, recommendation, or favoring by the United States government or Lawrence Livermore National Security, LLC. The views and opinions of authors expressed herein do not necessarily state or reflect those of the United States government or Lawrence Livermore National Security, LLC, and shall not be used for advertising or product endorsement purposes.

This work performed under the auspices of the U.S. Department of Energy by Lawrence Livermore National Laboratory under Contract DE-AC52-07NA27344. 


\title{
Reference guide to WPP version 2.0
}

\author{
N. Anders Petersson ${ }^{1} \quad$ Björn Sjögreen ${ }^{1}$
}

January 22, 2010

${ }^{1}$ Center for Applied Scientific Computing, Lawrence Livermore National Laboratory, PO Box 808, Livermore CA 94551. This work was performed under the auspices of the U.S. Department of Energy by Lawrence Livermore National Laboratory under contract DE-AC52-07NA27344. This is contribution LLNL-TR-XXXYYY 
Disclaimer This document was prepared as an account of work sponsored by an agency of the United States government. Neither the United States government nor Lawrence Livermore National Security, LLC, nor any of their employees makes any warranty, expressed or implied, or assumes any legal liability or responsibility for the accuracy, completeness, or usefulness of any information, apparatus, product, or process disclosed, or represents that its use would not infringe privately owned rights. Reference herein to any specific commercial product, process, or service by trade name, trademark, manufacturer, or otherwise does not necessarily constitute or imply its endorsement, recommendation, or favoring by the United States government or Lawrence Livermore National Security, LLC. The views and opinions of authors expressed herein do not necessarily state or reflect those of the United States government or Lawrence Livermore National Security, LLC, and shall not be used for advertising or product endorsement purposes. 


\section{Contents}

$\begin{array}{llr}1 & \text { Introduction } & 5\end{array}$

2 Getting started $\quad 6$

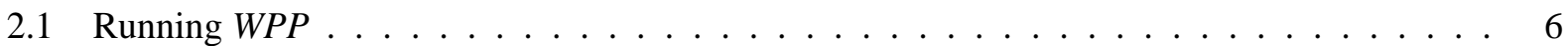

3 Coordinate system, units and the grid $\quad 8$

3.1 Geographic coordinates . . . . . . . . . . . . . . . . . . . 9

4 Sources, time-functions and grid sizes $\quad 11$

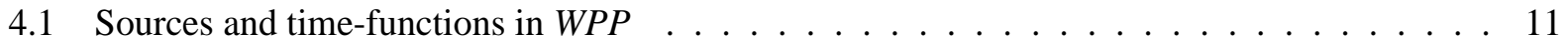

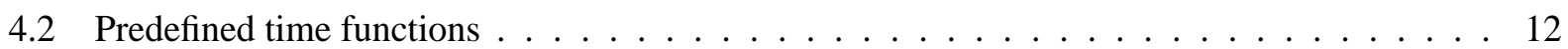

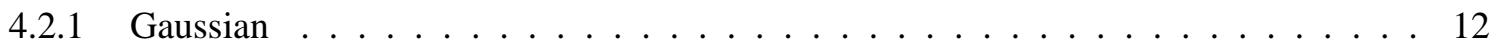

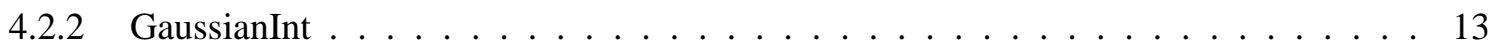

4.2 .3 Ricker . . . . . . . . . . . . . . . . . . . . 13

4.2 .4 RickerInt . . . . . . . . . . . . . . . . . . . . . . . 13

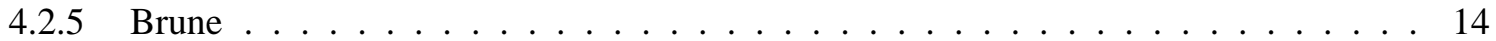

4.2 .6 BruneSmoothed . . . . . . . . . . . . . . . . . . . . . 14

4.2 .7 Liu . . . . . . . . . . . . . . . . . . . . . . 15

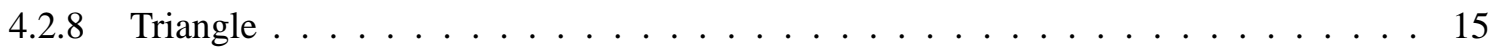

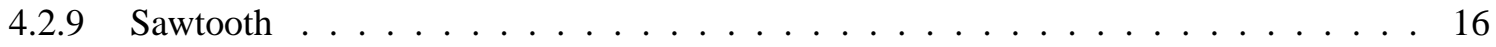

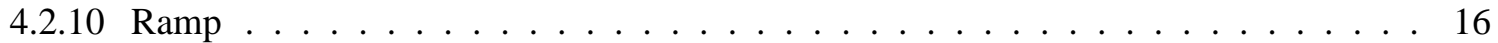

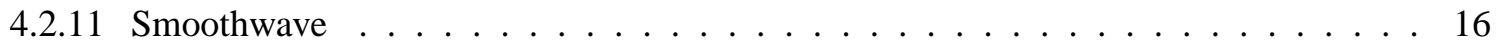

4.2 .12 VerySmoothBump . . . . . . . . . . . . . . . . 18

4.3 How fine does the grid need to be $\ldots \ldots \ldots \ldots \ldots \ldots$

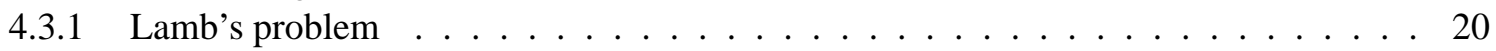

5 The material model $\quad 22$

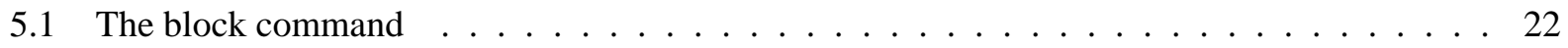

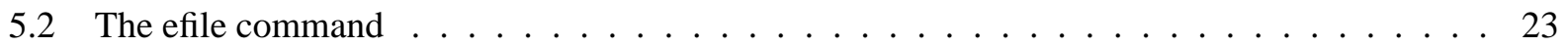

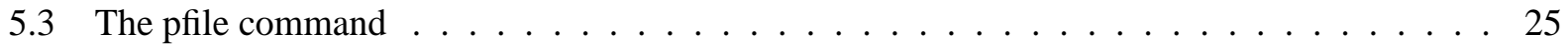

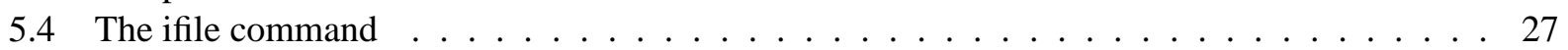

6 Topography $\quad 28$

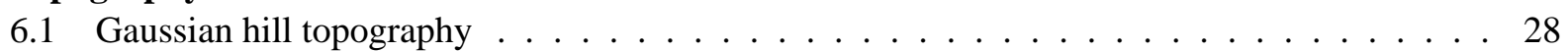

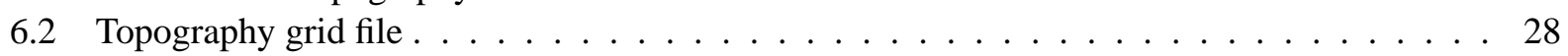

6.3 Etree topography . . . . . . . . . . . . . . . . . . . . . . . 29

7 Mesh refinement $\quad 31$ 
8 Output options $\quad 34$

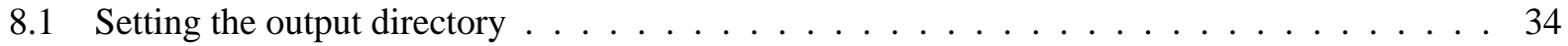

8.2 Time-history at a reciever station: the sac command . . . . . . . . . . . . 34

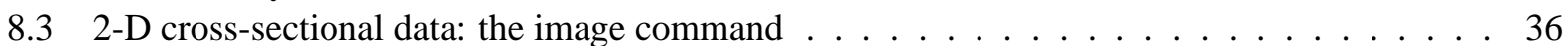

8.4 Generating a bird's eye view of the problem domain: the gmt command . . . . . . . . . 37

9 Examples $\quad 39$

9.1 Lamb's problem . . . . . . . . . . . . . . . . . . . . . . . . 39

9.2 Examples from Lifelines project 1A01: Validation of basin response codes . . . . . . . . 40

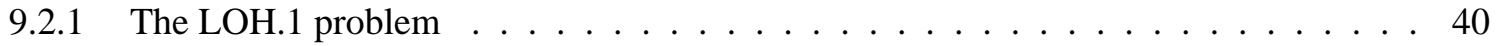

9.2 .2 The $\mathrm{LOH} .2$ problem $\ldots \ldots \ldots \ldots \ldots \ldots \ldots$

9.3 The Grenoble basin test case . . . . . . . . . . . . . . . . . . . . 43

9.4 Modeling the October 2007, Alum Rock earthquake . . . . . . . . . . . . . . . . . 46

10 Keywords in the input file $\quad 50$

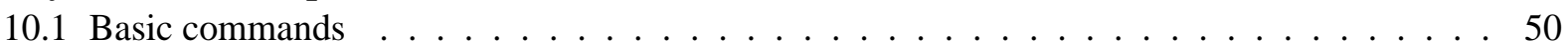

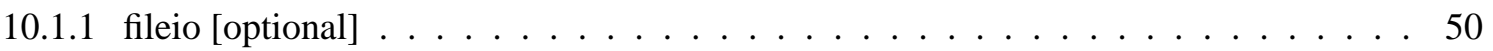

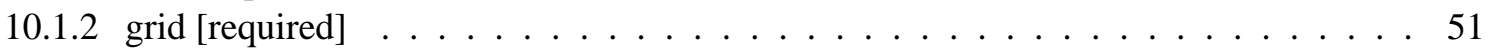

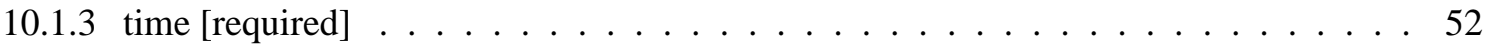

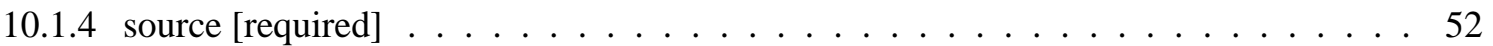

10.1 .5 prefilter $[$ optional $] \ldots \ldots \ldots \ldots \ldots$

10.2 The material model $[$ required $] \ldots \ldots \ldots \ldots \ldots$

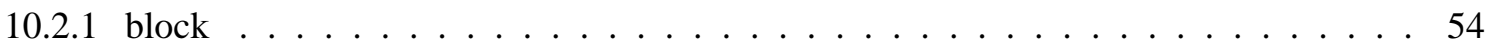

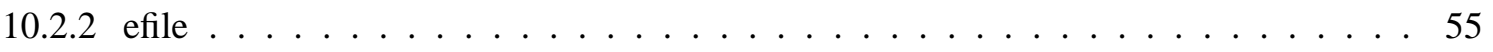

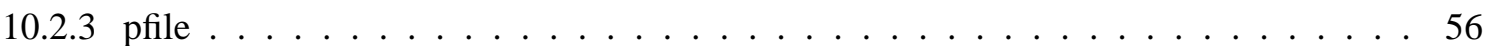

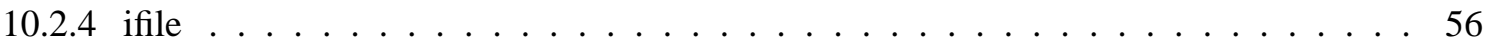

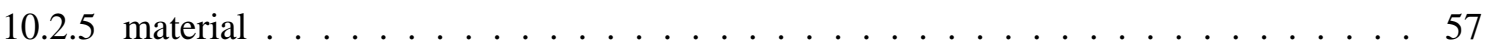

10.2 .6 globalmaterial $[$ optional] $\ldots \ldots \ldots \ldots \ldots \ldots \ldots$

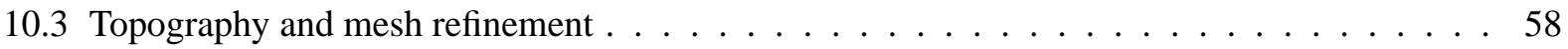

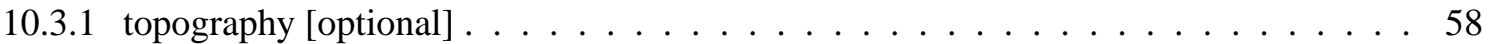

10.3 .2 refinement $[$ optional $] \ldots \ldots \ldots \ldots \ldots$

10.4 Output commands . . . . . . . . . . . . . . . . . . . . . . . . . 59

10.4 .1 sac $[$ optional] . . . . . . . . . . . . . . . . . . . . . . . . 59

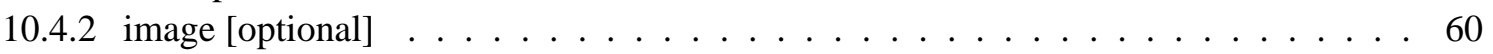

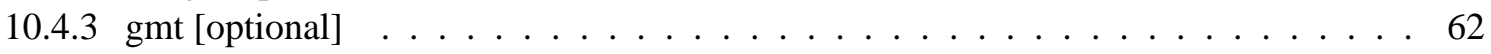

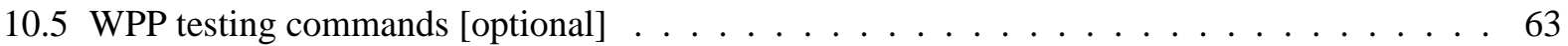

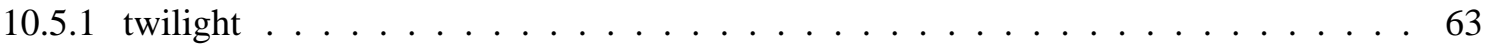

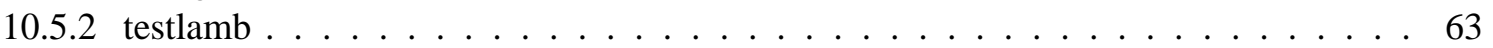

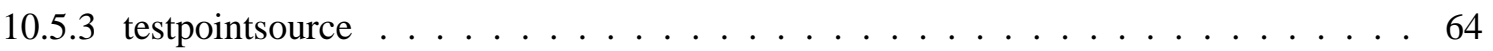

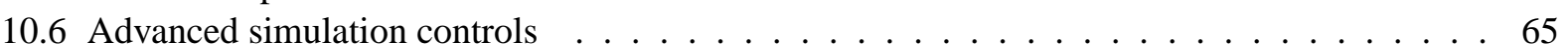

10.6 .1 supergrid $[$ optional $\ldots \ldots \ldots \ldots \ldots \ldots \ldots$

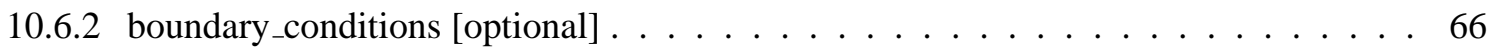

10.6 .3 developer $[$ optional $] \ldots \ldots \ldots \ldots$

11 File formats $\quad 68$

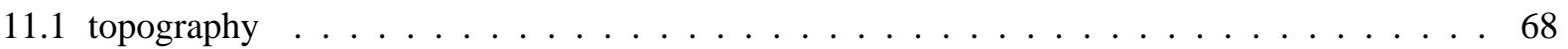

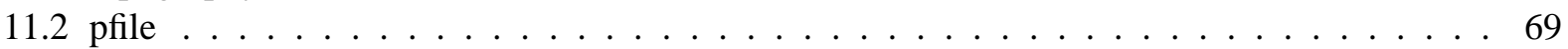

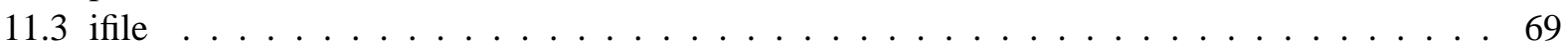




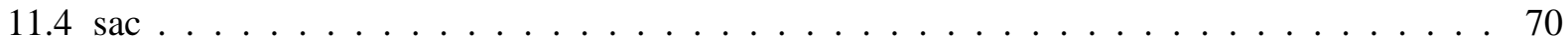

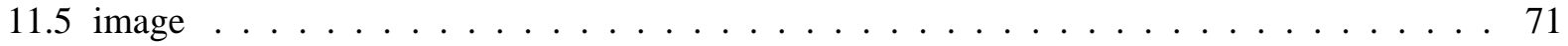

$\begin{array}{lll}\text { A Installing WPP } & \mathbf{7 3}\end{array}$

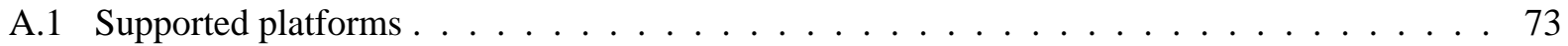

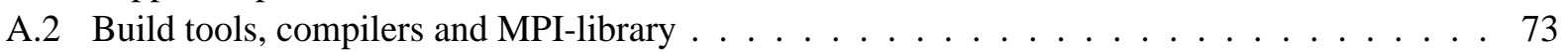

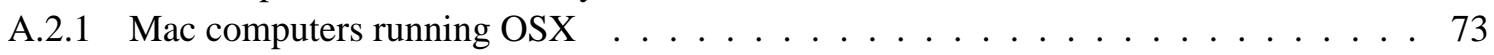

A.2.2 Linux machines . . . . . . . . . . . . . . . . . . 74

A.3 Directory structure . . . . . . . . . . . . . . . . . 74

A.4 Compiling and Linking WPP (without the cencalvm library) $\ldots \ldots \ldots \ldots$

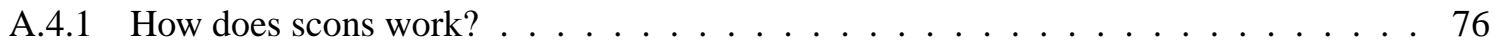

A.5 Installing cencalvm and its supporting libraries $\ldots \ldots \ldots \ldots$

B Testing the WPP installation $\quad 78$

B.1 Method of manufactured solutions $\ldots \ldots \ldots \ldots \ldots$

B.2 Lamb's problem . . . . . . . . . . . . . . . . . . . . . . . 79 


\section{Chapter 1}

\section{Introduction}

$W P P$ is a computer program for simulating seismic wave propagation on parallel machines. WPP solves the governing equations in second order formulation using a node-based finite difference approach. The basic numerical method is described in [9]. WPP implements substantial capabilities for 3-D seismic modeling, with a free surface condition on the top boundary, non-reflecting far-field boundary conditions on the other boundaries, point force and point moment tensor source terms with many predefined time dependencies, fully 3-D heterogeneous material model specification, output of synthetic seismograms in the $S A C$ [4] format, output of GMT [11] scripts for laying out simulation information on a map, and output of 2-D slices of (derived quantites of) the solution field as well as the material model.

Version 2.0 of WPP allows the free surface boundary condition to be imposed on a curved topography. For this purpose a curvilinear mesh is used near the free surface, extending into the computational domain down to a user specified level. The elastic wave equations and the free surface boundary conditions are discretized on the curvilinear mesh using the energy conserving technique described in [2]. A curvilinear mesh generator is built into WPP and the curvilinear mesh is automatically generated from the topography. Below the curvilinear grid, the elastic wave equation is discretized on Cartesian meshes, which leads to a more computationally efficient algorithm.

In version 2.0 of WPP, Cartesian local mesh refinement can be used to make the computational mesh finer near the free surface, where more resolution often is needed to resolve short wave lenghts in the solution, for example in sedimentary basins. The mesh refinement is performed in the vertical direction and each Cartesian grid is constructed from user specified refinement levels. In this approach, the grid size in all three spatial directions is doubled across each mesh refinement interface, leading to substantial savings in memory and computational effort. The energy conserving mesh refinement coupling method described in [10] is used to handle the hanging nodes along the refinement interface.

The examples subdirectory of the WPP source distribution contains several examples and validation tests. Many Matlab/octave scripts are provided in the tools directory.

Acknowledgments Many people have contributed to the development of WPP and we would like to thank (in no particular order) Artie Rodgers, Heinz-Otto Kreiss, Stefan Nilsson, Kathleen McCandeless, Hrvoje Tkalcic, Steve Blair, Daniel Appelö, and Caroline Bono. This work was enabled by financial support from a Laboratory Directed Research and Development (LDRD) project at Lawrence Livermore National Laboratory, as well as support from the OASCR program at the Office of Science at the Department of Energy. 


\section{Chapter 2}

\section{Getting started}

\subsection{Running WPP}

WPP can be run from the UNIX prompt or from a script. Normally WPP uses one argument: the name of the input file. The input file is an ASCII text file which contains a number of commands specifying the properties of the simulation, such as the dimensions of the computational domain, grid spacing, the duration of the simulation, the material properties, the source model, as well as the desired output. To improve readability of this document we have used the continuation character " $"$ to extend long commands to the subsequent line. There is however no support for continuation characters in WPP, so each command must be given on one (sometimes long) line in the input file.

Since WPP is a parallel code, it is required to be run under a parallel operating environment such as mpiexec, mpirun, or srun. For example,

shell> mpiexec -np 2 wpp test.in

tells WPP to read input from a file named test. in. Throughout this document we use the convention that input files have the file suffix . in, but WPP reads files with any extension.

Running on the Livermore Computing parallel linux clusters The srun command is currently used to run parallel jobs on LC machines. For example,

shell> srun -ppdebug $-\mathrm{n} 32$ wpp xxx.in

runs wpp on 32 processors on the debug parition using xxx.in as the input file. Note that the pdebug partition is intended for shorter jobs and is subject to both a CPU time limit and a limit on the number of processors per job. Jobs requiring more computer resources must be submitted through the batch system, currently using the msub command. Refer to the Livermore Computing web pages for detailed information (https://computing.llnl.gov).

Running on other platforms (Linux desktop/laptop): Depending on the MPI library, you may have to start an mpd daemon before starting your first parallel job (see mpich2-doc-user.pdf for more info). After that, wpp can be started using the mpirun command. For example,

shell> mpirun -np 2 wpp wpp.in

runs the wpp code on two processors, using wpp. in as the input file. 
version information (-v) Version information for the WPP executable can be obtained through $-\mathrm{v}$ flag:

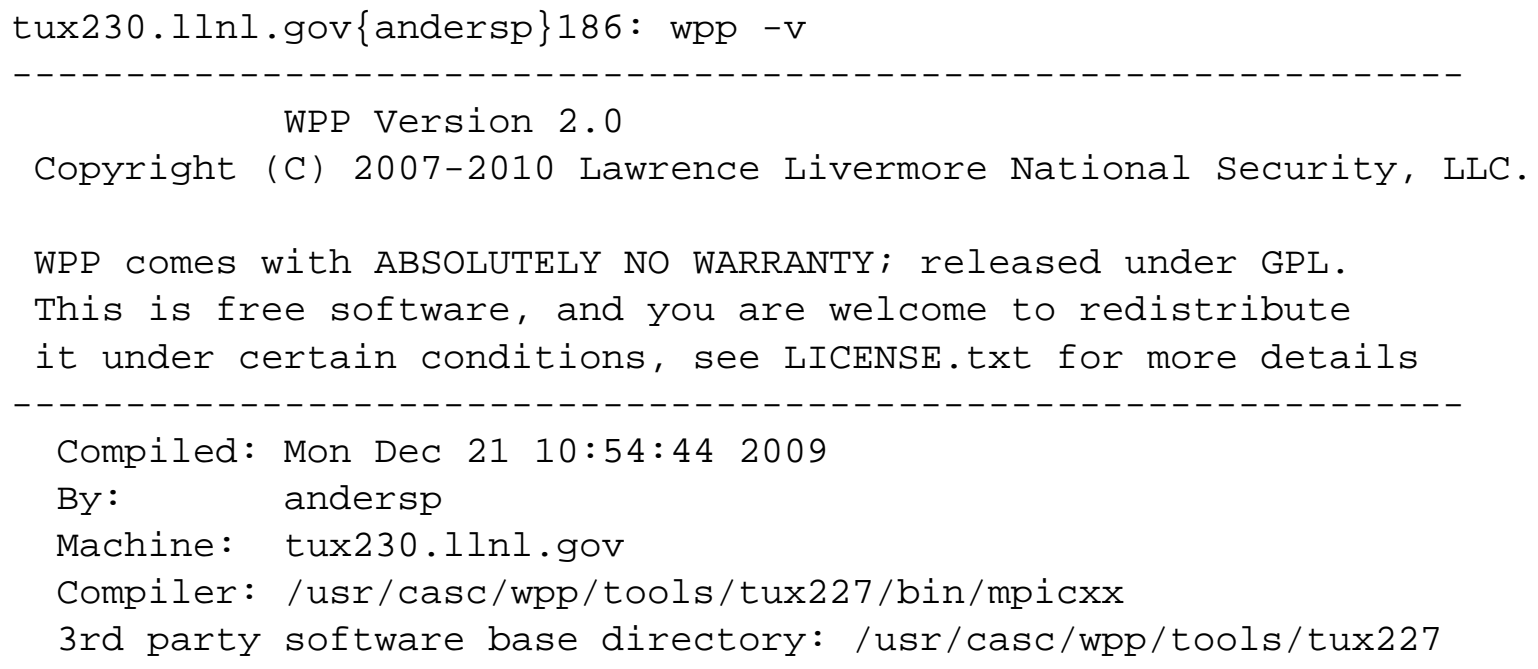

Note that the same information is by default printed to standard out at the beginning of every run. 


\section{Chapter 3}

\section{Coordinate system, units and the grid}

WPP uses a right-handed Cartesian coordinate system with the z-direction pointing downwards into the medium, see figure 3.1. WPP employs MKS (meters-kilograms-seconds) units; all distances (e.g., grid dimensions, spacing, and displacements) are in meters $(\mathrm{m})$, time is in seconds (s), seismic P- and S-wave velocities are in meters/second $(\mathrm{m} / \mathrm{s})$, densities are in kilogram/cubic meter $\left(\mathrm{kg} / \mathrm{m}^{3}\right)$, forces are in Newton $(\mathrm{N})$, and seismic moment (torque) is in Newton-meters (Nm). All angles (e.g. latitude, longitude, azimuth, strike, dip and rake) are in degrees.

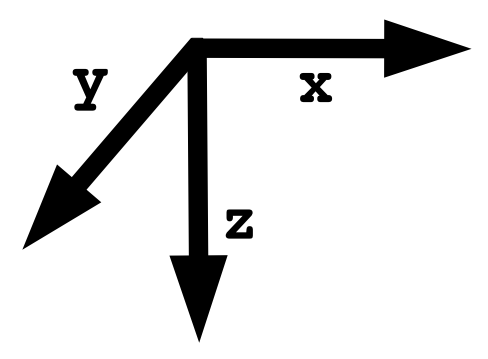

Figure 3.1: WPP uses a right handed coordinate system with the z-axis pointing downwards.

In WPP the computational domain is rectangular in the horizontal plane,

$$
0 \leq x \leq x_{\max }, \quad 0 \leq y \leq y_{\max }
$$

The topography surface

$$
z=\tau(x, y)
$$

defines the shape of the top surface in the vertical direction. WPP can also be run without topography, in which case $\tau(x, y)=0$. The computational domain is given by

$$
0 \leq x \leq x_{\max }, \quad 0 \leq y \leq y_{\max }, \quad \tau(x, y) \leq z \leq z_{\max } .
$$

The grid command in the input file specifies the extent of the computational domain and the grid size $h$. When mesh refinement is enabled, this is the grid size in the coarsest grid. The most obvious way of specifying the grid is by providing the number of grid points in each direction as well as the grid size,

grid $\mathrm{nx}=301 \mathrm{ny}=201 \mathrm{nz}=101 \mathrm{~h}=500.0$

This line gives a grid with grid size 500 meters, which extends $150 \mathrm{~km}$ in $x, 100 \mathrm{~km}$ in $y$ and $50 \mathrm{~km}$ in the $z$ direction. Alternatively, the grid can be specified by giving the spatial range in each of the three dimensions and explicitly specifying the grid spacing. For example, 
grid $x=30 e 3 \quad y=20 e 3 \quad z=10 e 3 \quad h=500.0$

results in a grid which spans 30,000 meters in $x, 20,000$ meters in $y$, and 10,000 meters in the $z$-direction. The grid spacing is 500 meters, which is used to compute the number of grid points in each direction: $\mathrm{nx}=61$, ny $=41$, and $\mathrm{nz}=21$, for a total of 52,521 grid points. Note that the number of grid points in the different directions will be rounded to the nearest integer value. For example

$$
n x=(\text { int }) 1.5+x / h,
$$

rounds $\mathrm{nx}$ to be the nearest integer value of $1+x / h$. The extent in the $x$-direction is thereafter adjusted to

$$
x=(n x-1) h .
$$

A corresponding procedure is performed in the other directions.

The third option is to give the spatial range in each of the three dimensions and specify the number of grid points in a particular direction:

grid $\mathrm{x}=30000 \mathrm{y}=20000 \mathrm{z}=10000 \mathrm{nx}=100$

In this case, the grid spacing is be computed as

$$
h=x /(n x-1)=303.03 .
$$

Note that no rounding needs to take place in this case, since $h$ is a floating point number. Given this value of $h$, ny and $\mathrm{nz}$ are computed using formulas corresponding to (3.2) giving ny $=34$ and $\mathrm{nz}=67$, for a total of 227,800 grid points. Again, the extents in the $y$ and $z$-directions are adjusted corresponding to (3.3). The syntax for the grid command is given in Section 10.1.2.

\subsection{Geographic coordinates}

WPP supports geographic coordinates as an alternative way of specifying spatial locations, see Figure 3.2. The location of the Cartesian coordinte system is specified in the grid command, and if no location is given the origin ( $x=0, y=0, z=0$ ) defaults to latitude 37 degrees (North), longitude -118 degrees (West), with a 135 degree azimuthal angle from North to the $x$-axis. The vertical coordinate is zero $(z=0)$ at mean sea level. The latitude $(\phi)$ and longitude $(\theta)$ are calculated using the approximative formulae (where lat, lon, and az are in degrees)

$$
\begin{aligned}
& \phi=\text { lat }+\frac{x \cos (\alpha)-y \sin (\alpha)}{M}, \quad \alpha=\mathrm{az} \frac{\pi}{180}, \\
& \theta=\text { lon }+\frac{x \sin (\alpha)+y \cos (\alpha)}{M \cos (\phi \pi / 180)}
\end{aligned}
$$

where $M=111319.5$ meters/degree. You can change the location and orientation of the grid by specifying the latitude and longitude of the grid origin, and the azimuthal angle between North and the $x$-axis. For example:

grid $h=500.0 \mathrm{x}=30000.0 \mathrm{y}=20000.0 \mathrm{z}=10000.0$ lat $=39.0$ lon=-117.0 az=150

sets the origin of the grid to latitude 39 degrees (North), longitude -117 degrees (West), and azimuthal angle 150 degrees. 


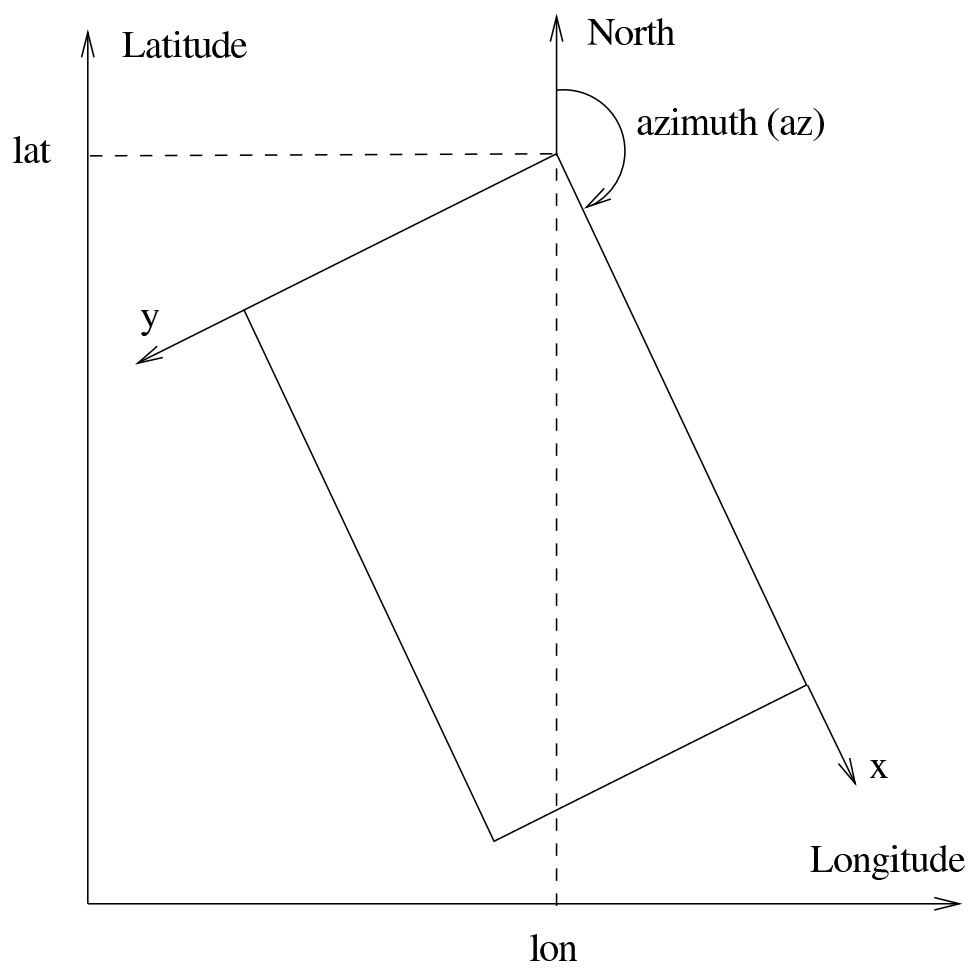

Figure 3.2: Geographical coordinates in WPP. 


\section{Chapter 4}

\section{Sources, time-functions and grid sizes}

\subsection{Sources and time-functions in WPP}

WPP solves the elastic wave equation in second order formulation,

$$
\begin{aligned}
\rho \mathbf{u}_{t t} & =\nabla \cdot \mathcal{T}+\mathbf{F}(\mathbf{x}, t), & \mathbf{x} \text { in } \Omega, t \geq 0 \\
\mathbf{u}(\mathbf{x}, 0) & =0, \quad \mathbf{u}_{t}(\mathbf{x}, 0)=0, & \mathbf{x} \text { in } \Omega
\end{aligned}
$$

where $\rho$ is the density, $\mathbf{u}(\mathbf{x}, t)$ is the displacement vector, and $\mathcal{T}$ is the stress tensor. The computational domain $\Omega$ is the box shaped region (3.1). By default, a free surface (zero traction) boundary condition is enforced along the top boundary,

$$
\mathcal{T} \cdot \mathbf{n}=0, \quad z=\tau(x, y), t \geq 0,
$$

where $\mathbf{n}$ is the unit normal of the $z=\tau(x, y)$ surface. A super-grid damping layer surrounds the computational domain on all other sides of the computational domain.

The forcing term $\mathbf{F}$ consists of a sum of point forces and point moment tensor source terms. For a point forcing we have

$$
\mathbf{F}(\mathbf{x}, t)=g\left(t, t_{0}, \omega\right) F_{0}\left(\begin{array}{c}
F_{x} \\
F_{y} \\
F_{z}
\end{array}\right) \delta\left(\mathbf{x}-\mathbf{x}_{\mathbf{0}}\right),
$$

where $\mathbf{x}_{0}=\left(x_{0}, y_{0}, z_{0}\right)$ is the location of the point force in space, and $g\left(t, t_{0}, \omega\right)$ is the time function, with offset time $t_{0}$ and frequency parameter $\omega$. The $\left(F_{x}, F_{y}, F_{z}\right)^{T}$ vector holds the Cartesian components of the force vector, which is scaled by the force amplitude $F_{0}$.

For a moment tensor source we have

$$
\mathbf{F}(\mathbf{x}, t)=g\left(t, t_{0}, \omega\right) M_{0} \mathcal{M} \cdot \nabla \delta\left(\mathbf{x}-\mathbf{x}_{\mathbf{0}}\right), \quad \mathcal{M}=\left(\begin{array}{ccc}
M_{x x} & M_{x y} & M_{x z} \\
M_{x y} & M_{y y} & M_{y z} \\
M_{x z} & M_{y z} & M_{z z}
\end{array}\right) .
$$

In this case the seismic moment of the moment tensor is $M_{0}$, otherwise the notation is the same as for a point force. Note that the moment tensor always is symmetric. A convenient way of specifying moment sources is by using the dip, strike and rake angles (see Section 10.1.4 for syntax) defined in Aki and Richards [1]. In this case, the total seismic moment $\sum M_{0}[\mathrm{Nm}]$ is related to the moment magnitude by the formula

$$
M_{W}=\frac{2}{3}\left[\log _{10}\left(\sum M_{0}\right)-9.1\right] \text {. }
$$


After parsing all source commands in an input file, WPP outputs the moment magnitude using this formula.

For moment tensor sources, the function $g(t)$ is called the moment history time function, while its time derivative $g^{\prime}(t)$ is known as the moment rate time function. WPP calculates the displacements of the motion corresponding to the moment history time function $g(t)$. However, since the material properties are independent of time, the equations solved by WPP also govern the velocities when the time function is replaced by $g^{\prime}(t)$, i.e., the corresponding moment rate time function. For example, if the solution calculated with the GaussianInt time function represents the displacements of the motion, the solution calculated with the Gaussian time function corresponds to the velocities of the same motion. Hence, if you are primarily interested in calculating velocities, you can reduce the amount of post processing by using the corresponding moment rate time function in the source term(s).

Note that most first order formulation codes (such as E3D) are based on the velocity-stress formulation of the elastic wave equation. These codes use the moment rate time function (i.e., the Gaussian time function in the above example) and solve for the velocities of the motion.

In WPP the forcing is specified in the input file using the source command. There needs to be at least one source command in the input file in order for anything to happen during the simulation. Complicated source mechanisms can be described by having many source commands in the input file. An example with one source command is:

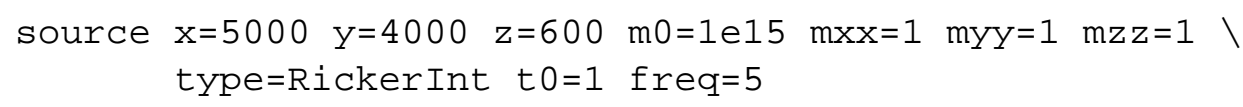

which specifies an isotropic source (explosion) at the point $\mathbf{r}_{0}=(5000,4000,600)$ with amplitude $10^{15}$ $\mathrm{Nm}$, using the RickerInt time function with offset time $t_{0}=1 \mathrm{~s}$ and frequency parameter $\omega=5 \mathrm{~Hz}$. This command sets the off-diagonal moment tensor elements $\left(M_{x y}, M_{x z}\right.$ and $\left.M_{y z}\right)$ to zero (which is the default value).

Note that it is not necessary to place the sources exactly on grid points. The discretization of the source terms is second order accurate for any location within the computational domain.

\subsection{Predefined time functions}

The source time function can be selected from a set of predefined functions described below. All functions start from zero $\left(\lim _{t \rightarrow-\infty} g\left(t, t_{0}, \omega\right)=0\right)$ and tend to a contant terminal value, $\lim _{t \rightarrow \infty} g\left(t, t_{0}, \omega\right)=g_{\infty}$. In seismic applications, $g_{\infty} \neq 0$ always corresponds to solving for the displacements of the motion, because the solution will tend to a non-zero steady state solution for large times. This solution corresponds to the final displacements due to a seismic event. When $g_{\infty}=0$, the solution will always tend to zero for large times, as is expected from the velocities or accelerations of the motion due to a seismic event.

The Gaussian and the Triangle functions integrate to one $\left(\int_{-\infty}^{\infty} g\left(t, t_{0}, \omega\right) d t=1\right)$, while the Sawtooth, Smoothwave, and Ricker functions integrate to zero and have maximum amplitude one. The RickerInt function is the time-integral of the Ricker function and integrates to zero. The GaussianInt, Brune, BruneSmoothed, and Liu functions tend to one $\left(\lim _{t \rightarrow \infty} g\left(t, t_{0}, \omega\right)=1\right)$.

The Triangle, Sawtooth, Ramp, Smoothwave, Brune, BruneSmoothed, Liu and VerySmoothBump functions are identically zero for $t<t_{0}$, so they will give reasonable simulation results if $t_{0} \geq 0$. However, the Gaussian, GaussianInt, Ricker, and RickerInt functions are centered around $t=t_{0}$ with exponentially decaying tails for $t<t_{0}$. Hence $t_{0}$ must be positive and of the order $\mathcal{O}(1 / \omega)$ to avoid incompatibilty problems with the initial conditions. We recommend choosing $t_{0}$ such that $g\left(0, t_{0}, \omega\right) \leq 10^{-8}$ for these functions.

\subsubsection{Gaussian}

$$
g\left(t, t_{0}, \omega\right)=\frac{\omega}{\sqrt{2 \pi}} e^{-\omega^{2}\left(t-t_{0}\right)^{2} / 2}
$$



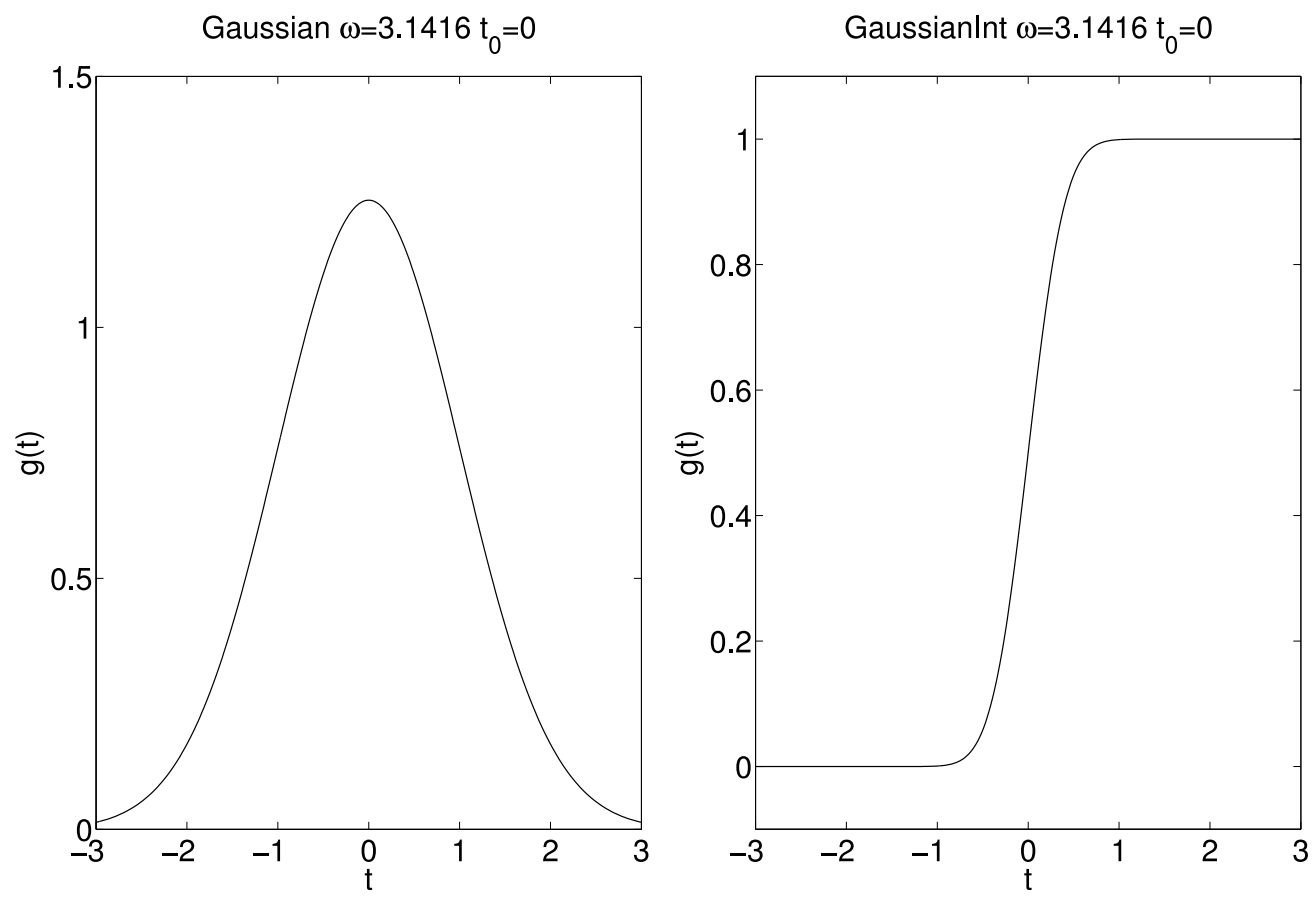

Figure 4.1: Gaussian (left) and GaussianInt (right) with $\omega=\pi$ and $t_{0}=0$.

Note that the spread of the Gaussian function (often denoted $\sigma$ ) is related to $\omega$ by $\sigma=1 / \omega$. A plot of the Gaussian time-function is shown in Figure 4.1.

\subsubsection{GaussianInt}

The GaussianInt function is often used in earthquake modeling since it leads to a permanent displacement.

$$
g\left(t, t_{0}, \omega\right)=\frac{\omega}{\sqrt{2 \pi}} \int_{-\infty}^{t} e^{-\omega^{2}\left(\tau-t_{0}\right)^{2} / 2} d \tau
$$

GaussianInt is the time-integral of the Gaussian. A plot of the GaussianInt time-function is shown in Figure 4.1.

\subsubsection{Ricker}

$$
g\left(t, t_{0}, \omega\right)=\left(2 \pi^{2} \omega^{2}\left(t-t_{0}\right)^{2}-1\right) e^{-\pi^{2} \omega^{2}\left(t-t_{0}\right)^{2}} .
$$

A plot of the Ricker time-function is shown in Figure 4.2.

\subsubsection{RickerInt}

$$
g\left(t, t_{0}, \omega\right)=\left(t-t_{0}\right) e^{-\pi^{2} \omega^{2}\left(t-t_{0}\right)^{2}} .
$$

RickerInt is the time integral of the Ricker function, and is proportional to the time-derivative of the Gaussian function. The RickerInt function is sometimes used in seismic exploration simulations. Since the RickerInt function tends to zero for large times, it does not lead to any permanent displacements. A plot of the RickerInt time-function is shown in Figure 4.2. 

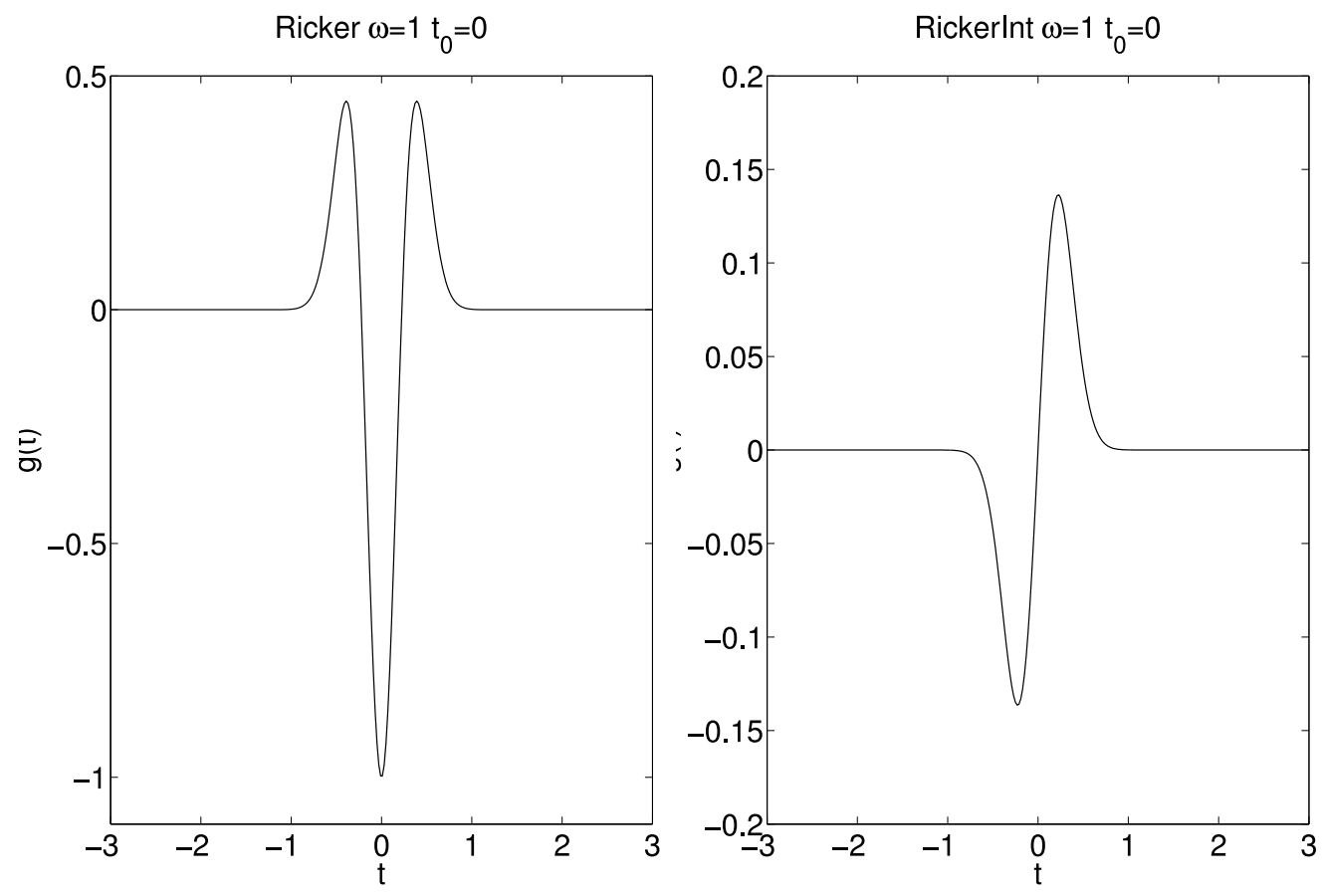

Figure 4.2: Ricker (left) and RickerInt (right) with $\omega=1$ and $t_{0}=0$.

\subsubsection{Brune}

The Brune function has one continuous derivative but its second derivative is discontinuous at $t=t_{0}$,

$$
g\left(t, t_{0}, \omega\right)= \begin{cases}0, & t<t_{0}, \\ 1-e^{-\omega\left(t-t_{0}\right)}\left(1+\omega\left(t-t_{0}\right)\right), & t \geq t_{0} .\end{cases}
$$

The Brune function is often used in earthquake modeling.

\subsubsection{BruneSmoothed}

The BruneSmoothed function has three continuous derivatives at $t=t_{0}$, but is otherwise close to the Brune function:

$$
g\left(t, t_{0}, \omega\right)= \begin{cases}0, & t<t_{0}, \\ 1-e^{-\omega\left(t-t_{0}\right)}\left[1+\omega\left(t-t_{0}\right)+\frac{1}{2}\left(\omega\left(t-t_{0}\right)\right)^{2}\right. & \\ \left.-\frac{3}{2 x_{0}}\left(\omega\left(t-t_{0}\right)\right)^{3}+\frac{3}{2 x_{0}^{2}}\left(\omega\left(t-t_{0}\right)\right)^{4}-\frac{1}{2 x_{0}^{3}}\left(\omega\left(t-t_{0}\right)\right)^{5}\right], & 0<\omega\left(t-t_{0}\right)<x_{0}, \\ 1-e^{-\omega\left(t-t_{0}\right)}\left(1+\omega\left(t-t_{0}\right)\right), & \omega\left(t-t_{0}\right)>x_{0} .\end{cases}
$$

The parameter is fixed to $x_{0}=2.31$. Plots of the Brune and BruneSmoothed time-functions are shown in Figure 4.3. Since the BruneSmoothed function has three continuous derivatives, it generates less high frequency noise. Compared to the Brune function, the BruneSmoothed function gives better accuracy at a given grid resolution 

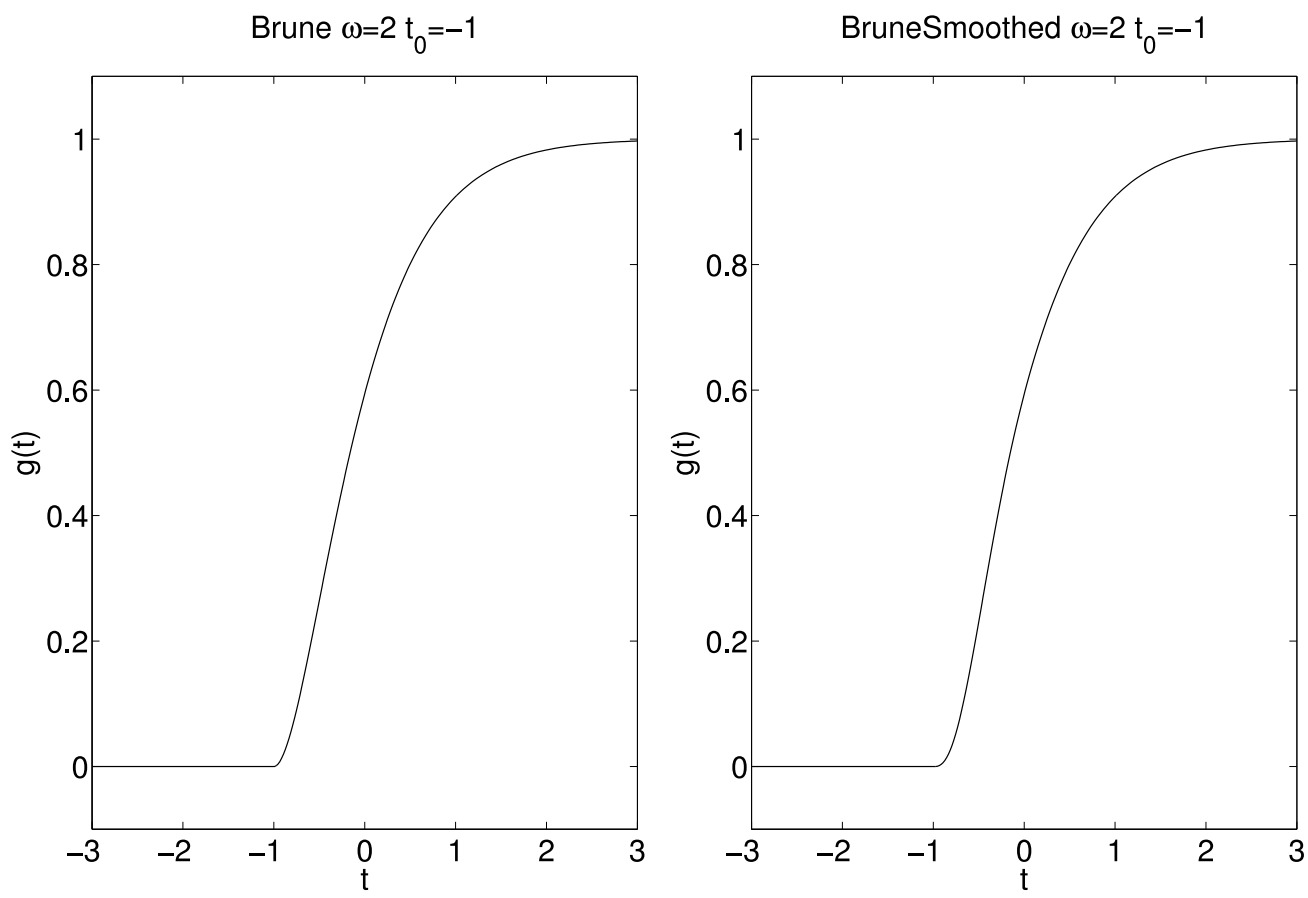

Figure 4.3: Brune (left) and BruneSmoothed (right) with $\omega=2$ and $t_{0}=-1$.

\subsubsection{Liu}

This function was given in a paper by Liu et al., [7]. It is defined by

$$
g\left(t, t_{0}, \omega\right)=\left\{\begin{array}{rr}
0, & t \leq t_{0}, \\
C\left[0.7\left(t-t_{0}\right)+\frac{1.2}{\pi} \tau_{1}-\frac{1.2}{\pi} \tau_{1} \cos \left(\frac{\pi\left(t-t_{0}\right)}{2 \tau_{1}}\right)\right. & \\
C\left[t-t_{0}-0.3 \tau_{1}+\frac{1.2}{\pi} \tau_{1}-\frac{0.7}{\pi} \tau_{1} \sin \left(\frac{\pi\left(t-t_{0}\right)}{\tau_{1}}\right)\right], & t_{0}<t \leq \tau_{1}+t_{0}, \\
\left.+\frac{0.3}{\pi} \tau_{2} \sin \left(\frac{\pi\left(t-t_{0}\right)}{\tau_{2}}\right) \tau_{1}\right) & \\
C\left[0.3\left(t-t_{0}\right)+1.1 \tau_{1}+\frac{1.2}{\pi} \tau_{1}\right), & \tau_{1}+t_{0}<t \leq 2 \tau_{1}+t_{0}, \\
\left.+\frac{0.3}{\pi} \tau_{2} \sin \left(\frac{\pi\left(t-t_{0}-\tau_{1}\right)}{\tau_{2}}\right)\right], & 2 \tau_{1}+t_{0}<t \leq \tau+t_{0}, \\
1, \quad t>\tau+t_{0} .
\end{array}\right.
$$

The parameters are given by $\tau=2 \pi / \omega, \tau_{1}=0.13 \tau, \tau_{2}=\tau-\tau_{1}$, and $C=\pi /\left(1.4 \tau_{1} \pi+1.2 \tau_{1}+0.3 \tau_{2} \pi\right)$. The Liu function resembles the Brune function, but the rise is somewhat steeper for small $t-t_{0}$, see Figure 4.4.

\subsubsection{Triangle}

For $t_{0}<t<t_{0}+1 / \omega$,

$$
g\left(t, t_{0}, \omega\right)=\frac{16 \omega}{\pi^{2}}\left[\sin \left(\pi \omega\left(t-t_{0}\right)\right)-\frac{\sin \left(3 \pi \omega\left(t-t_{0}\right)\right)}{9}+\frac{\sin \left(5 \pi \omega\left(t-t_{0}\right)\right.}{25}-\frac{\sin \left(7 \pi \omega\left(t-t_{0}\right)\right)}{49}\right],
$$




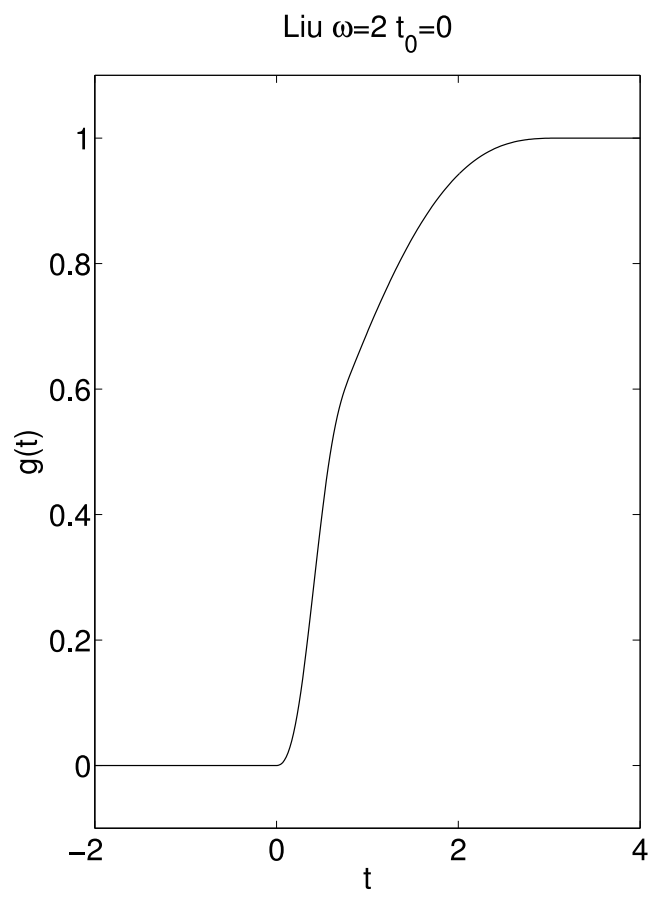

Figure 4.4: Liu time function with $\omega=2$ and $t_{0}=0$.

with $g\left(t, t_{0}, \omega\right)=0$ elsewhere. A plot of the Triangle time-function is shown in Figure 4.5.

\subsubsection{Sawtooth}

For $t_{0}<t<t_{0}+1 / \omega$

$$
g\left(t, t_{0}, \omega\right)=\frac{8}{\pi^{2}}\left[\sin \left(2 \pi \omega\left(t-t_{0}\right)\right)-\frac{\sin \left(6 \pi \omega\left(t-t_{0}\right)\right)}{9}+\frac{\sin \left(10 \pi \omega\left(t-t_{0}\right)\right)}{25}-\frac{\sin \left(14 \pi \omega\left(t-t_{0}\right)\right)}{49}\right],
$$

with $g\left(t, t_{0}, \omega\right)=0$ elsewhere. A plot of the Sawtooth time-function is shown in Figure 4.5.

\subsubsection{Ramp}

$$
g\left(t, t_{0}, \omega\right)= \begin{cases}0, & t<t_{0} \\ 0.5\left(1-\cos \left(\pi\left(t-t_{0}\right) \omega\right)\right), & t_{0} \leq t \leq t_{0}+1 / \omega \\ 1, & t>t_{0}+1 / \omega\end{cases}
$$

A plot of the Ramp time-function is shown in Figure 4.6.

\subsubsection{Smoothwave}

For $t_{0}<t<t_{0}+1 / \omega$,

$$
\begin{aligned}
g\left(t, t_{0}, \omega\right)=\frac{2187}{8}\left(\omega\left(t-t_{0}\right)\right)^{3}-\frac{10935}{8}\left(\omega\left(t-t_{0}\right)\right)^{4}+ & \frac{19683}{8}\left(\omega\left(t-t_{0}\right)\right)^{5} \\
& -\frac{15309}{8}\left(\omega\left(t-t_{0}\right)\right)^{6}+\frac{2187}{4}\left(\omega\left(t-t_{0}\right)\right)^{7},
\end{aligned}
$$

with $g\left(t, t_{0}, \omega\right)=0$ elsewhere. A plot of the Smoothwave time-function is shown in Figure 4.6. 

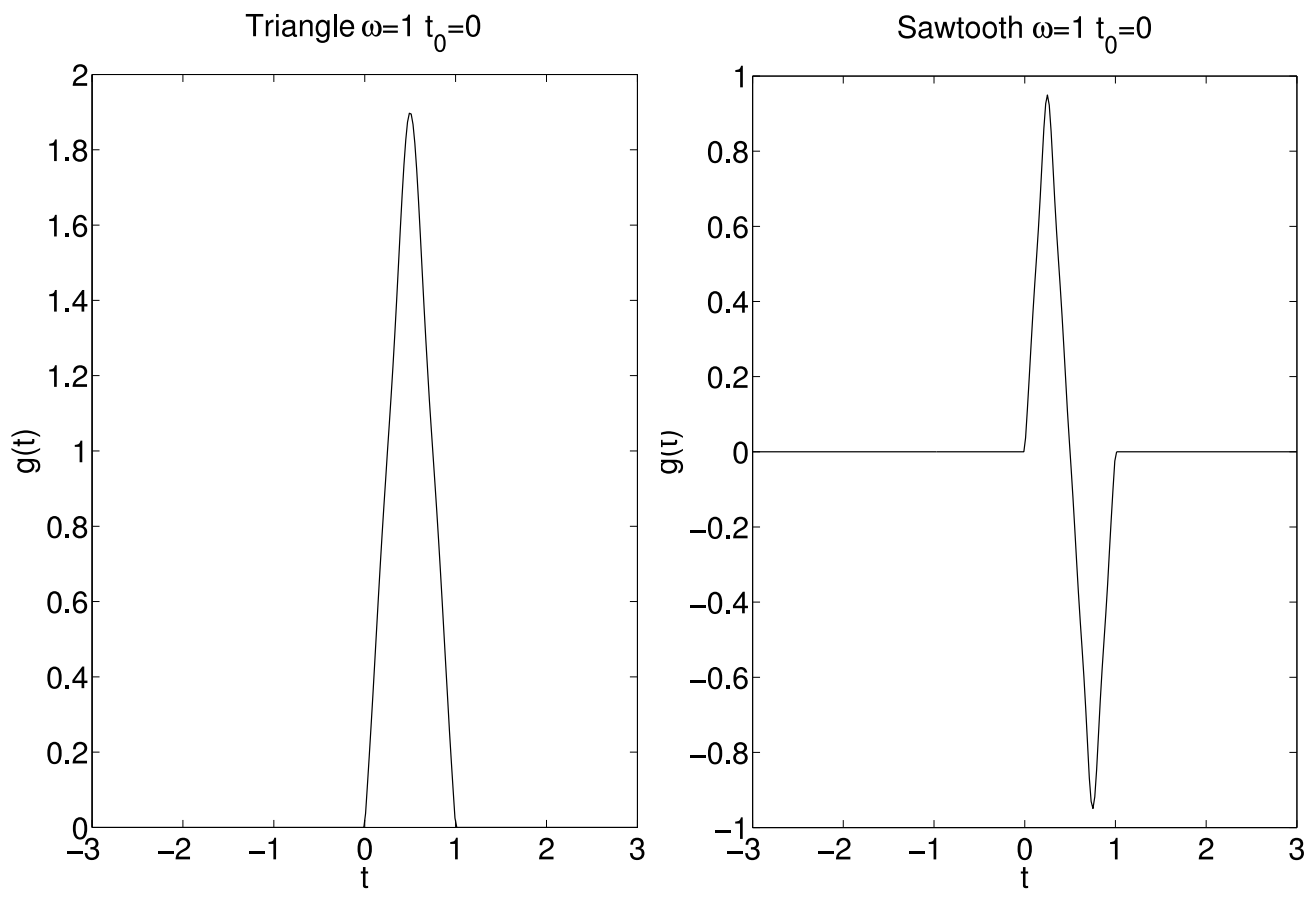

Figure 4.5: Triangle (left) and Sawtooth (right) with $\omega=1$ and $t_{0}=0$.
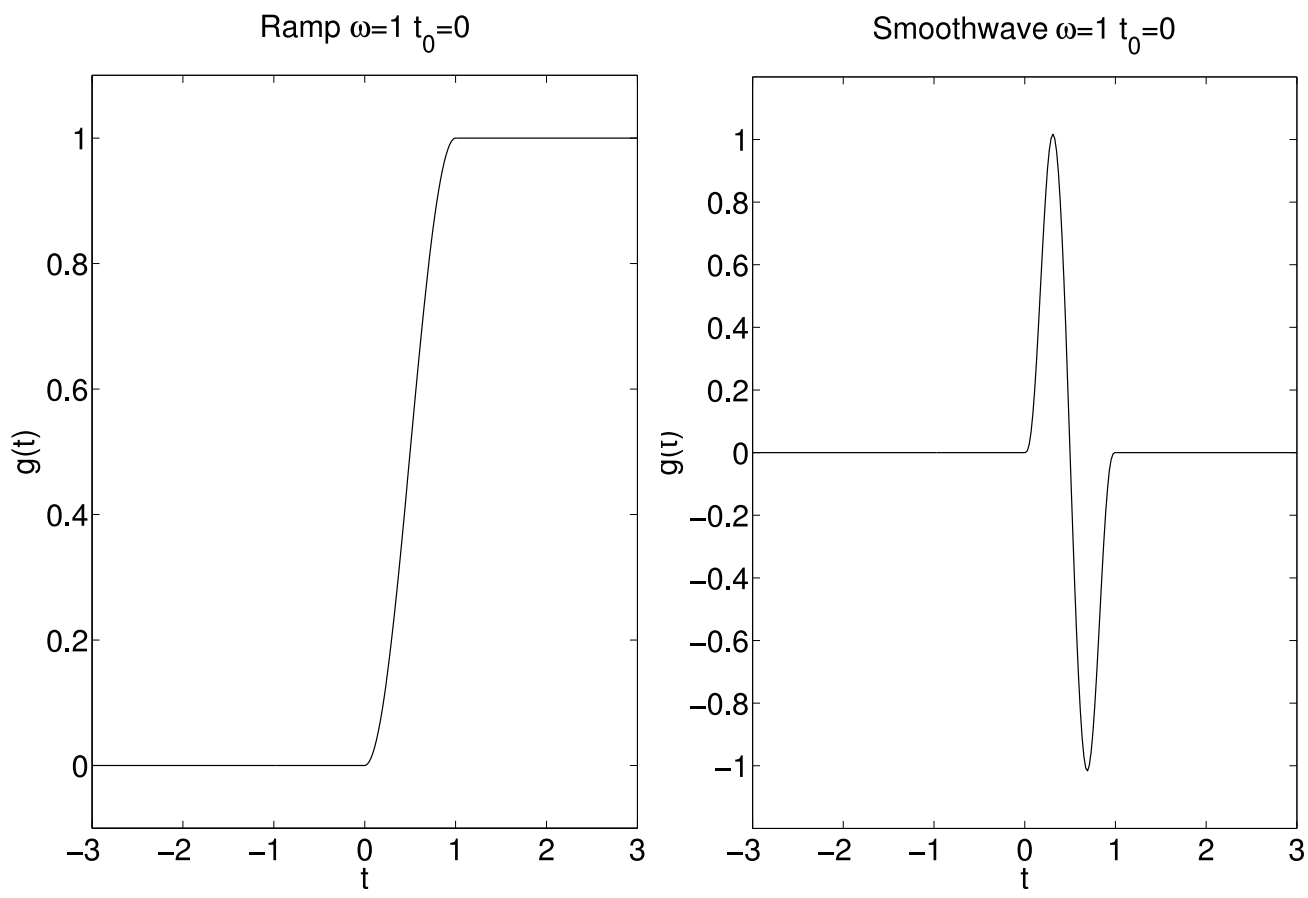

Figure 4.6: Ramp (left) and Smoothwave (right) with $\omega=1$ and $t_{0}=0$. 


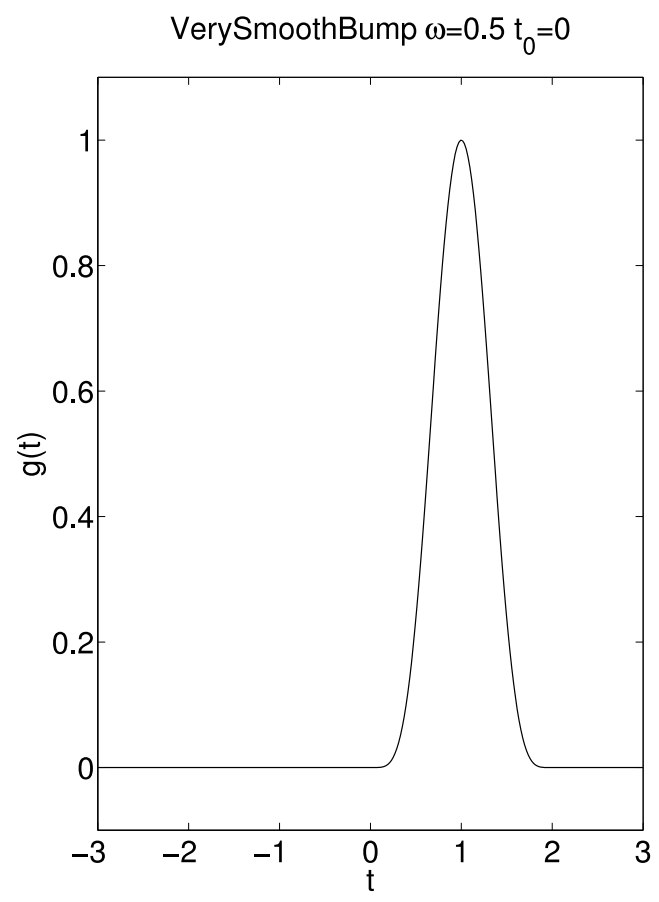

Figure 4.7: VerySmoothBump with $\omega=0.5$ and $t_{0}=0$.

\subsubsection{VerySmoothBump}

$$
g\left(t, t_{0}, \omega\right)= \begin{cases}0, & t<t_{0}, \\ -1024\left(\omega\left(t-t_{0}\right)\right)^{10}+5120\left(\omega\left(t-t_{0}\right)\right)^{9}-10240\left(\omega\left(t-t_{0}\right)\right)^{8} & \\ \quad+10240\left(\omega\left(t-t_{0}\right)\right)^{7}-5120\left(\omega\left(t-t_{0}\right)\right)^{6}+1024\left(\omega\left(t-t_{0}\right)\right)^{5}, & t_{0} \leq t \leq t_{0}+1 / \omega, \\ 0, & t>t_{0}+1 / \omega .\end{cases}
$$

A plot of the VerySmoothBump time-function is shown in Figure 4.7.

\subsection{How fine does the grid need to be?}

The most difficult parameter to choose when preparing the input file is probably the grid size $h$. It is extremely important to use a grid size which is sufficiently small, because you must resolve the waves which are generated by the source. On the other hand you don't want to use an unnecessarily small grid size, because both the computational demand and the memory requirements increase with decreasing grid size.

The number of grid points per shortest wavelength, $P$, is a normalized measure of how well a solution is resolved on the computational grid. Since the shear waves have the lowest velocities and a shorter wave length than the compressional waves, the shortest wave length $L_{\min }$ can be estimated by

$$
L_{\min }=\frac{\min V_{s}}{f_{\max }},
$$

where $V_{s}$ is the shear velocity of the material and $f_{\max }$ is the largest significant frequency in the time 


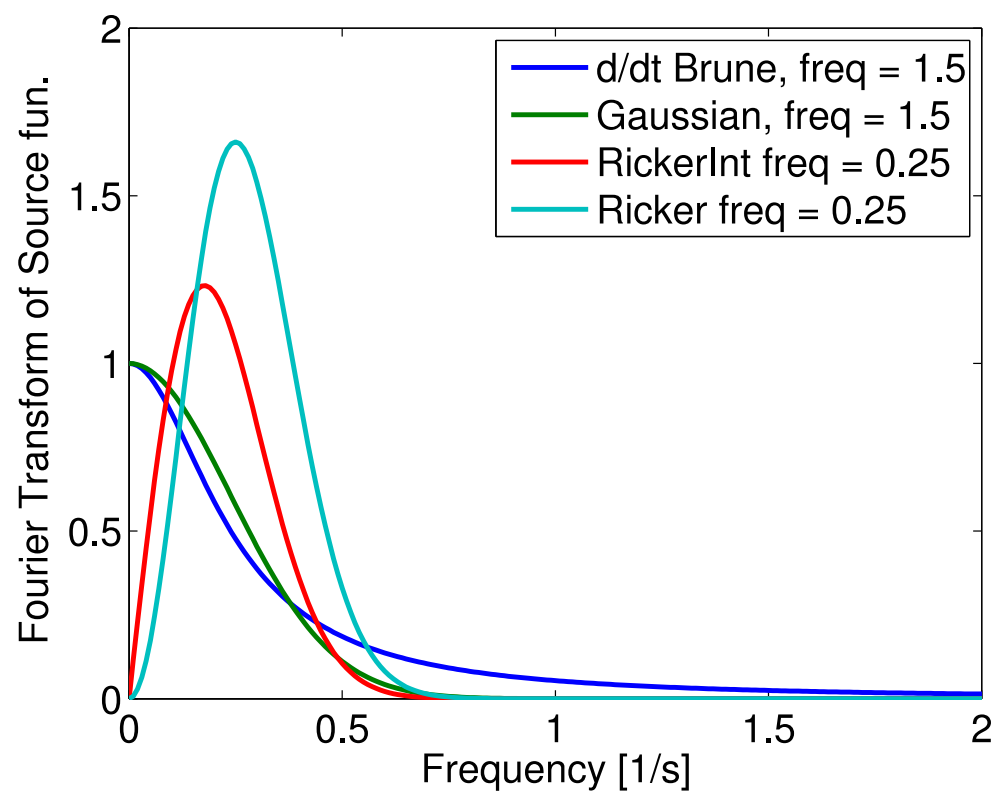

Figure 4.8: Magnitude of the Fourier transform of the derivative of the Brune (dark blue), the Gaussian (green), the RickerInt (red), and the Ricker (light blue) time-functions. Here freq=1.5 for the Gaussian and the derivative of the Brune function, and $f$ req $=0.25$ for Ricker and RickerInt.

function $g(t)$. Hence the number of grid points per wave length equals $L_{\min } / h$, which is given by

$$
P=\frac{\min V_{s}}{h f_{\max }} .
$$

Note that $h$ needs to be made smaller to maintain the same value of $P$ if either $V_{s}$ is decreased or if the frequency is increased. In formula (4.1), $\min V_{s}$ is found from the material properties and $h$ is determined by the input grid specification. The frequencies present in the solution are determined by the frequencies present in the time function(s) in the source term(s).

Figure 4.8 displays the absolute values of the Fourier transforms of the functions Gaussian, RickerInt, Ricker, and the time derivative of the Brune function. Inspection of the mathematical definitions of the Gaussian and Brune functions shows that the freq parameter specifies the angular frequency for these functions. The relation between the fundamental frequency $f_{0}$ and the freq parameter is given by

$$
f_{0}= \begin{cases}\text { freq }, & \text { for Ricker, RickerInt, and VerySmoothBump, } \\ \text { freq } /(2 \pi), & \text { for Liu, Brune, BruneSmoothed, Gaussian, and GaussianInt. }\end{cases}
$$

The plots in Figure 4.8 were made with frequency parameter freq $=0.25$ for the Ricker and RickerInt functions and frequency parameter freq $=1.5$ for the Gaussian and $d / d t$ (Brune) functions. Hence, $f_{0} \approx$ 0.25 for all functions in Figure 4.8. Note that the Fourier transform of the Brune function decays much slower than the other functions for high frequencies. This is due to its lack of smoothness at $t=t_{0}$.

It is the upper power (highest significant) frequency in the time function that shall be used in (4.1) to estimate the number of grid points per wave length. For practical purposes $f_{\max }$ is defined as the frequency above which the amplitude of the Fourier transform falls below $5 \%$ of its max value. We have

$$
f_{\text {max }} \approx\left\{\begin{array}{l}
2.5 f_{0}, \quad \text { Ricker, RickerInt, Gaussian time functions }, \\
4 f_{0}, \quad \text { Brune time function. }
\end{array}\right.
$$



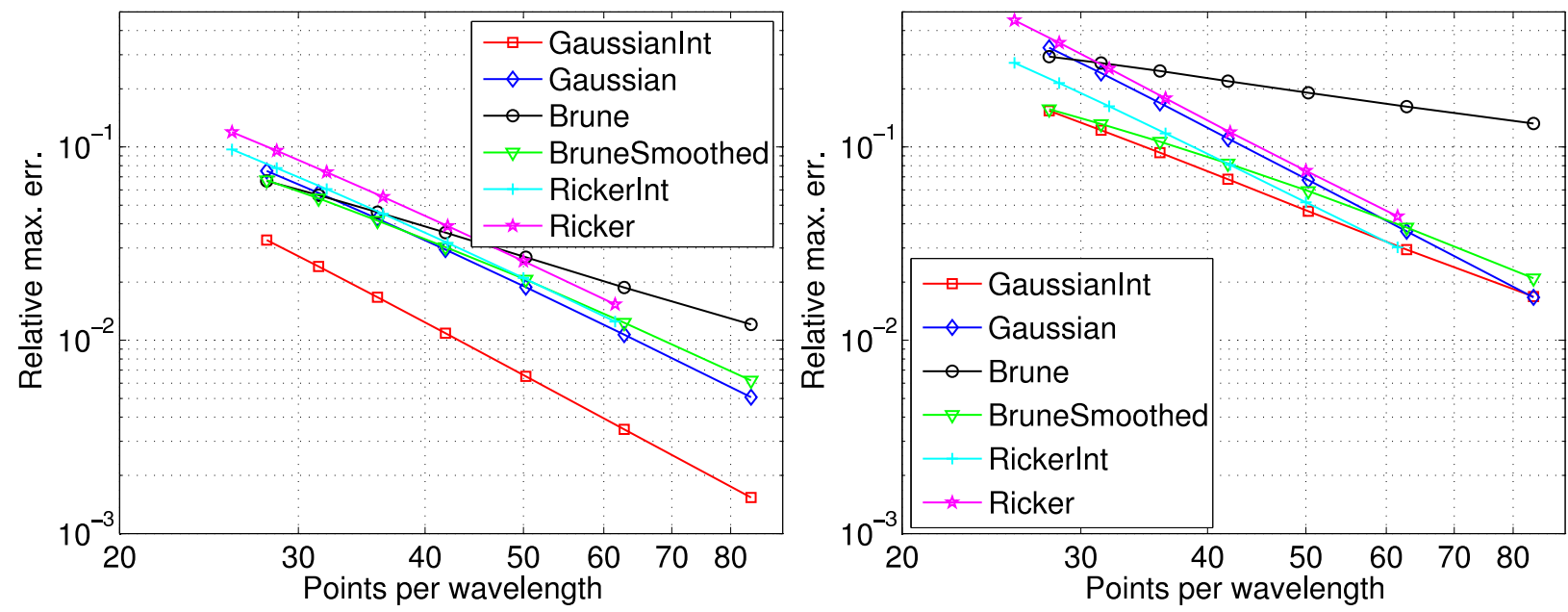

Figure 4.9: Relative errors for different source functions 10 (left) and $50 \mathrm{~km}$ (right) from the source. For the Brune time function the error decays much slower than for the other time functions. Here, the number of points per wavelength $(P)$ was based on the fundametal frequency $f_{0}$ instead of $f_{\max }$, so the values of $P$ should be divided by 2.5 for GaussianInt, Gaussian, RickerInt, and Ricker. For Brune, $P$ should be divided by 4 .

In other words, simulations using the Brune function are harder to resolve on the grid and need much more grid points to give reliable results.

Our experience is that $W P P$ gives accurate results for

$$
P \geq 15
$$

but the exact number depends on the distance between the source and the reciever. Note that the relation between the fundamental frequency $f_{0}$ and the upper power frequency $f_{\max }$ in (4.3) is very important. For other time functions, $f_{\max }$ can be estimated using the matlab/octave scripts fenplot and ftfcnplot in the tools directory. A lower number for $P$ can be used in many practical situations, for example when there are significant uncertainties in the material properties.

\subsubsection{Lamb's problem}

We now compute solutions to Lamb's problem in a material with $V_{p}=\sqrt{3} \mathrm{~km} / \mathrm{s}, V_{s}=1 \mathrm{~km} / \mathrm{s}$ and the density $1000 \mathrm{~kg} / \mathrm{m}^{3}$. The solution is forced downward with amplitude $\mathrm{f} z=5 \mathrm{e} 13 \mathrm{~N}$ and with a time function centered at time $t 0=25 \mathrm{~s}$. For various time functions the solution is recorded at receivers 10 and $50 \mathrm{~km}$ from the source. At the recievers the relative error

$$
\frac{\left\|u_{\text {exact }}(t)-u_{\text {computed }}(t)\right\|_{\infty}}{\left\|u_{\text {exact }}(t)\right\|_{\infty}}
$$

in the horizontal component is computed and plotted in Figure 4.9. In these calculations, the grid size was held constant and the frequency parameter freq was varied. Note that the reported number of points per wavelength was based on the fundamental frequency $f_{0}$ instead of $f_{\max }$, so the values of $P$ should be reduced according to (4.3)

From Figure 4.9 we see that for all of the time functions, except the Brune function, there is a decrease in error inversely proportional to the square of the number of points per wavelength. The errors are larger 
for the Brune function since its spectrum decays much slower due to its discontinuous second derivative at $t=t_{0}$. The difference in the error levels between the left and the right sub-figures are due to the fact that errors in the numerical solution accumulate as the solution propagates away from the source. For a single harmonic wave, and a second order accurate finite difference method, the number of points per wavelength required to achieve a certain error is proportional to the square root of the number of wavelengths the wave propagates (see Chapter 3 in [5] for a detailed discussion). Thus, to get the same accuracy at five times the distance from the source, we need to use about $\sqrt{5} \approx 2.24$ times more points per wave length. This could be achived by reducing the grid size by a factor 2.24 in each direction, resulting in a factor of 11.1 times more grid points and an increase in CPU time by a factor 25 . 


\section{Chapter 5}

\section{The material model}

The material model in WPP is defined by the values of the density, $\rho$, the compressional velocity, $V_{p}$, and the shear velocity, $V_{s}$, at each grid point. These values can be specified by the block command ( $\left.\S 5.1\right)$, the efile command ( $\S 5.2$ ), the pfile command ( $\S 5.3$ ), the ifile command ( $\S 5.4)$, or by a combination of them.

Note that WPP uses a single layer of ghost points outside the computational domain (as defined by the grid command). The material properties must therefore be defined for the computational domain padded by one layer of ghost points. Note, however, that the material model does not need to be defined above the free surface. Material properties at those points are instead assigned by extrapolation from the interior of the domain.

It is important to note that the order within the material commands (block, pfile, efile, and ifile) does matter (unlike all other commands) in that the priority of the material command increase towards the end of the input file. Hence, a material command in the input file can be completely or partially overridden by subsequent material commands.

In the block, pfile, and ifile commands, material properties are assigned based on the depth below the free surface. This means that the internal material model depends on the topography, but the material properties along the free surface will always be the same, independently of the topography model. For the efile command, material properties are defined as functions of elevation relative to mean sea level $(z=0)$. Here the topography information is embedded in the material description. If you combine the efile command with a planar topography, a linear mapping is constructed before the material properties are assigned to the top (finest) Cartesian grid. The properties at the free surface are thus mapped to the top grid surface $(z=0)$, and the bottom grid surface with $z=z_{N}$ is assigned material properties for elevation $-z_{N}$. Elevation values at intermediate grid points follow from the linear mapping. Subsequent (coarser) Cartesian grids are not effected by this mapping procedure.

\subsection{The block command}

The block command can be used to specify material properties in rectangular volumes of the computational domain, either with constant values or linear vertical gradients. By combining the block command with the sub-region options we can define a material model composed of three layers:

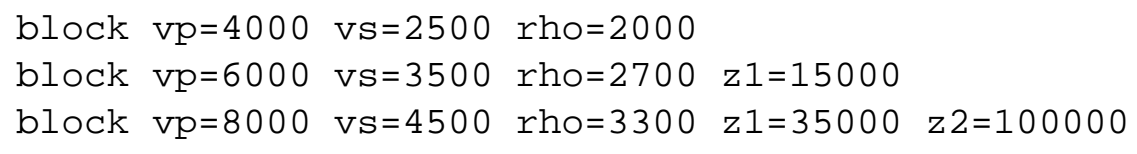

In this case the top layer has a thickness of $15 \mathrm{~km}$, the middle layer $20 \mathrm{~km}$ and the lower layer $65 \mathrm{~km}$. Because these block commands do not specify horizontal coordinates, the values extend to the grid boundaries in both 
horizontal directions. To add a box shaped inclusion of a new material we could add the following line

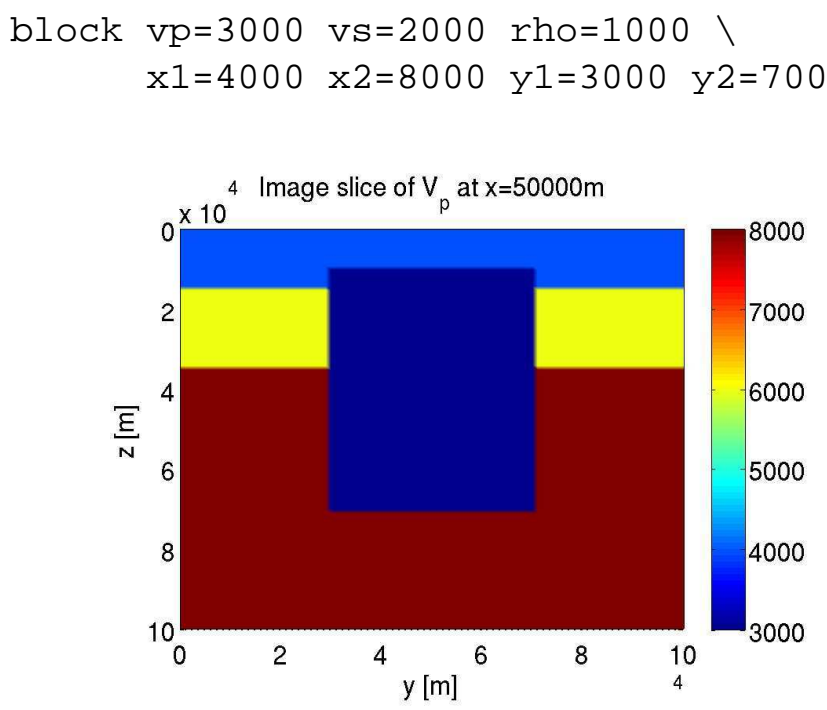

$z 1=10000 \quad z 2=70000$

Figure 5.1: Examples of material models specified with the block command.

To the left in Figure 5.1 an image slice of $V_{p}$ through $x=50,000$ is displayed.

The following example combines several block commands used to generate the material model displayed to the right in Figure 5.1:

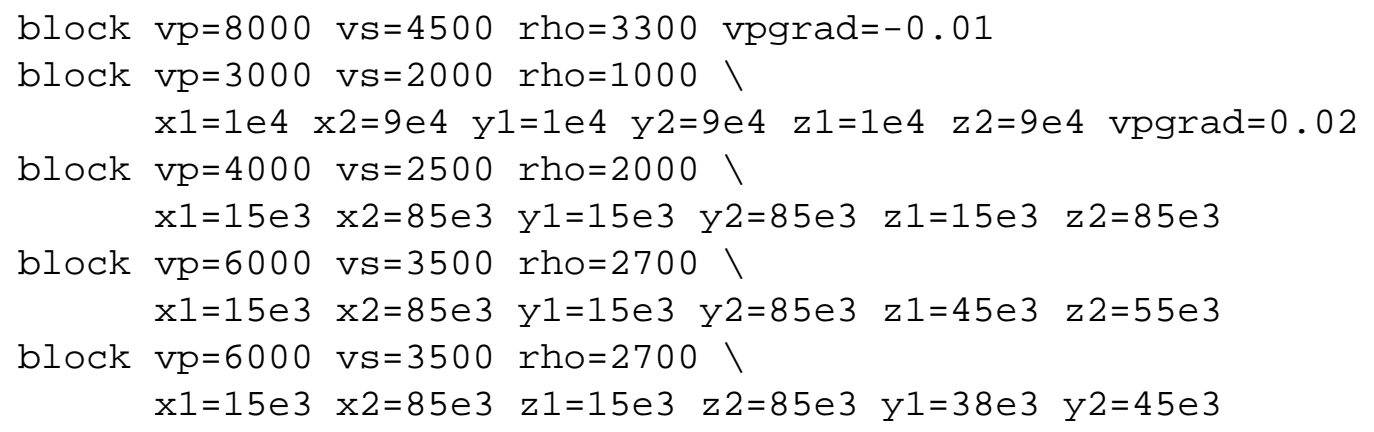

\subsection{The efile command}

The efile command is used to read in material properties from an etree database file. Etree databases use an oct-tree data structure which allows material properties to be represented with finer spatial resolution near the surface. Topography and bathymetry information is included in the database. The same etree database file can be used independently of the grid size, so there is no need to have a one-to-one mapping between the etree model and the computational grid. Unfortunately, it takes a major effort to develop an etree database file, and we currently only have access to material data for Northern California and the extended San Francisco bay area. This model was developed by the USGS and can be accessed from http://www.sf06simulation.org/geology/velocitymodel. Be aware that the database is rather large and can take a very long time to download. The geographical extent of the etree model is given in Table 5.1, which also is shown on a map in Figure 5.2.

In the etree database, material properties are stored as functions of geographic coordinates (latitude, longitude, elevation). WPP uses formulas (3.4)-(3.5) to determine that information for each grid point before 


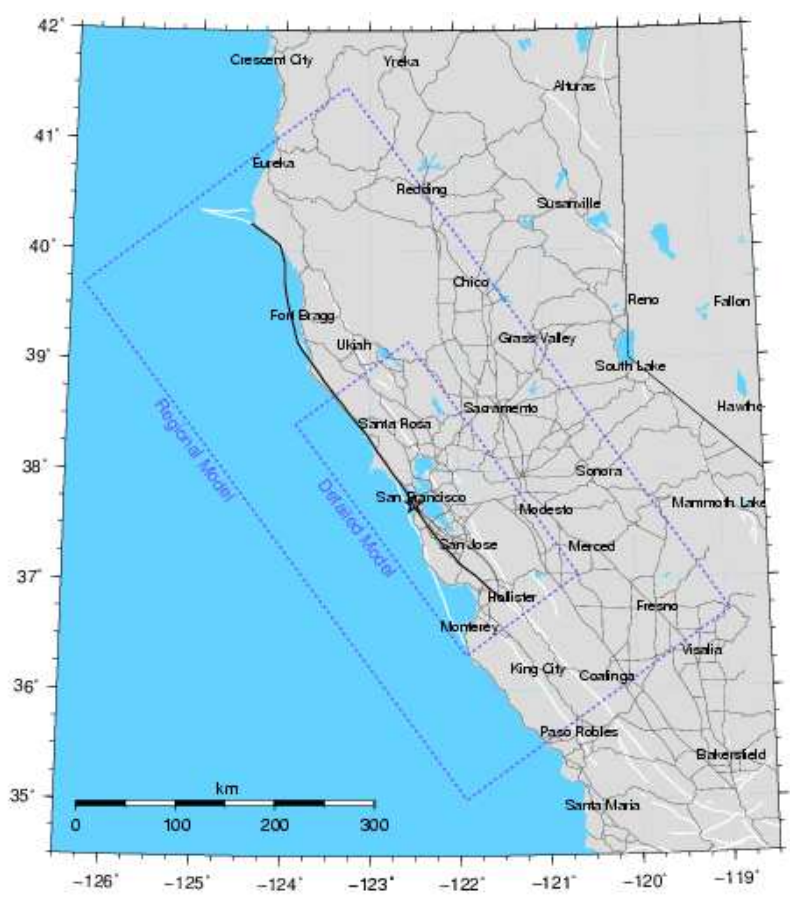

Figure 5.2: The geographical extent of the etree models for Northern California and the San Francisco bay area.

\begin{tabular}{|l|l|l|}
\hline \multicolumn{3}{|c|}{ Detailed Model } \\
\hline Corner & Longitude & Latitude \\
\hline SE & -120.64040 & 37.04718 \\
\hline SW & -121.91833 & 36.31746 \\
\hline NW & -123.85736 & 38.42426 \\
\hline NE & -122.56127 & 39.17461 \\
\hline
\end{tabular}

\begin{tabular}{|l|l|l|}
\hline \multicolumn{3}{|c|}{ Regional Model } \\
\hline Corner & Longitude & Latitude \\
\hline SE & -118.944514 & 36.702176 \\
\hline SW & -121.930857 & 35.009018 \\
\hline NW & -126.353173 & 39.680558 \\
\hline NE & -123.273199 & 41.48486 \\
\hline
\end{tabular}

Table 5.1: Geographic extent (NAD27 projection) for the central California velocity models. Both models are defined down to $45 \mathrm{~km}$ depth. See http://www.sf06simulation.org/geology/velocitymodel for details. 
it obtains the material properties from the data base. Internally to WPP, the cencalvm software library is used to query the etree database. Hence, before the efile command can be used, the corresponding software libraries must be installed and WPP must be configured to use them, see Section A.

It is important to note the bounds of the geographical region in the database. Assuming the computational domain is contained within the bounds of the database, it is easy to set up the material model in the input file:

grid $\mathrm{x}=100 \mathrm{e} 3 \mathrm{y}=100 \mathrm{e} 3 \mathrm{z}=40 \mathrm{e} 3$ lat=38.0 lon=-121.8 az=144 $\mathrm{h}=1000$

efile etree $=/ \mathrm{p} /$ lscratchd/andersp/USGSBayAreaVM-08.3.0.etree

To verify that the computational domain is inside the etree data base, we recommend checking the geographic coordinates on map before the simulation is started. We often use the google earth program for this purpose. In the case when the computational domain is larger than the region covered by the efile, a block command can be used to assign material properties to grid points outside of the efile region:

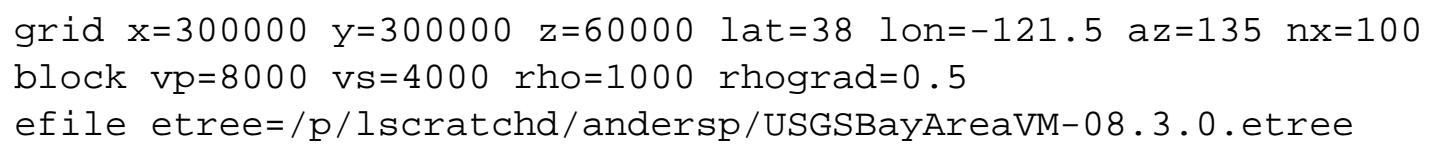

However, sharp jumps in material properties can lead to significant artificial scattering of seismic waves. In some cases, better results can be obtained by reducing the size of the computational domain to match the extent of the etree region.

To enable use of the extended SF model, the extended etree file must also be downloaded and then added to the efile command line (names have been shortened for better readability):

efile etree=USGSBayAreaVM.etree xetree=USGSBayAreaVMExt.etree

\subsection{The pfile command}

The pfile command can be used to assign material properties based on depth profiles on a lattice. A pfile contains the values of the model features (P-velocity, S-velocity, density, and Q-factors) as function of depth at points on an equally spaced latitude-longitude lattice. The number of grid points in the depth direction needs to be the same for all profiles, but the grid spacing does not need to be uniform and can also be different for each profile. Material discontinuities can be represented by two material values for the same depth value. Furthermore, layers which only occur in a subset of the profiles can be tapered to zero thickness in the remaining profiles. This is handled by introducing multiple data points with the same depth and material values in a profile.

The lattice of the pfile does not need to have any relation to the computational mesh used in WPP and is often much coarser. The material properties in the computational mesh are assigned values using Gaussian averaging between the nearest $N_{G} \times N_{G}$ profiles in the latitude-longitude plane and linear interpolation in the depth direction. Let the grid point have longitude $\theta$, latitude $\phi$ and depth $d$. Material properties are first linearly interpolated in the depth direction along each profile and then averaged in the latitude-longitude plane. The number of points in the Gaussian averaging, $N_{G}$, is assigned by the user in the pfile command. For example, the following line in the input file makes WPP read a pfile named material.ppmod:

pfile filename=material.ppmod vsmin=1000 vpmin=1732 smoothingsize=4

The optional vsmin and vpmin keywords are used to assign minimum threshold values for the $P$ - and $S$-velocities, respectively. smoothingsize $=4$ means that $N_{G}=4$ in the Gaussian averaging. A larger value of $N_{G}(\geq 5)$ is particularily useful to avoid staircasing imprints when the computational grid is much 

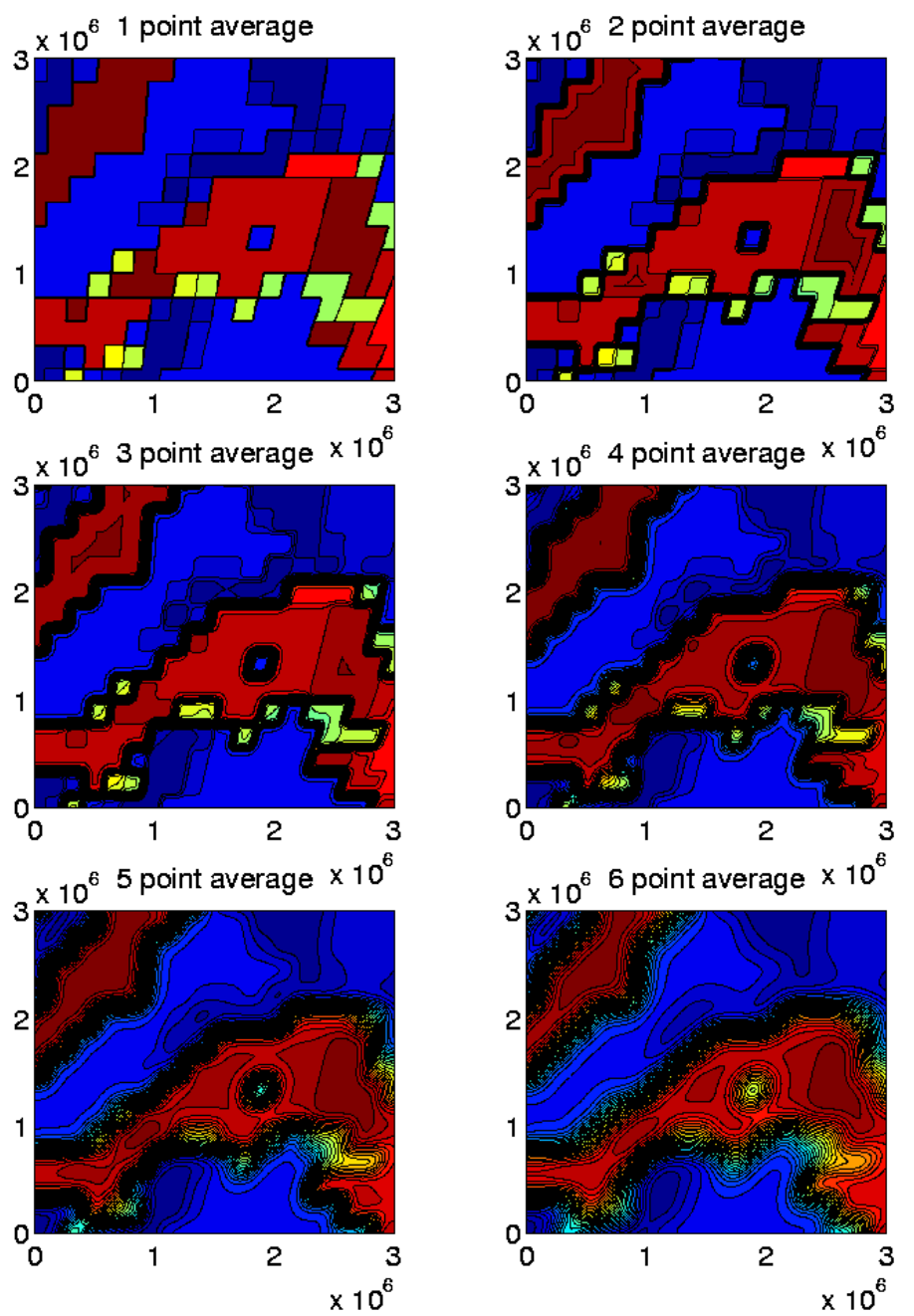

Figure 5.3: The smoothingsize parameter can be used to average out abrupt variations in the horizontal plane (constant depth) in a coarse pfile material model. 
finer than the pfile lattice, see Figure 5.3. smoothingsize can be set to any number greater than or equal to one.

When $N_{G}$ is odd, the Gaussian averaging starts by finding the closest grid point on the latitude-longitude lattice, $\left(\phi_{i}, \theta_{j}\right)$. The material property $c\left(\rho, V_{p}, V_{s}\right.$, etc. $)$ is assigned by the formula

$$
c(\phi, \theta)=\frac{\sum_{m=i-Q}^{i+Q} \sum_{n=j-Q}^{j+Q} c_{m, n} \omega_{m, n}}{\sum_{m=i-Q}^{i+Q} \sum_{n=j-Q}^{j+Q} \omega_{m, n}}, \quad Q=\frac{N_{G}-1}{2},
$$

where the weights are given by

$$
\omega_{m, n}=e^{-\left[\left(\phi_{m}-\phi\right)^{2}+\left(\theta_{n}-\theta\right)^{2}\right] / \alpha^{2}}, \quad \alpha=\frac{N_{G} \Delta_{l a t}}{2 \sqrt{-\log 10^{-6}}},
$$

and the grid size in the latitude-longitude lattice is $\Delta_{\text {lat }}$. This choice of $\alpha$ makes the weights $\omega_{m, n}<10^{-6}$ for points that are further from $\left(\phi_{m}, \theta_{n}\right)$ than $N_{G} \Delta_{l a t} / 2$, which justifies the truncation of the series in (5.1). A similar procedure is used for even values of $N_{G}$, but in this case the averaging formula (5.1) is centered around the nearest cell center on the latitude-longitude lattice.

Data files for the pfile command are in ASCII text format, see Section 11.2.

\subsection{The ifile command}

The ifile command reads a file holding the depth to material interface surfaces. The material properties between each pair of material surfaces must be defined by the material command. The depth must be non-negative. Zero depth corresponds to the topography. Material surfaces are specified on a regular lattice in gegraphic coordinates. The unit for depth is meters, while latitude and longitude are in degrees. The ifile command may be combined with other material specifications and it is not necessary that the lattice in geographic coordinates covers the horizontal extent of the computational domain.

Let $N_{\text {mat }} \geq 1$ material surfaces be known at longitudes

$$
\phi_{i}, \quad i=1,2, \ldots, N_{l o n}
$$

and latitudes

$$
\theta_{j}, \quad j=1,2, \ldots, N_{l a t},
$$

Note that the latitudes and the longitudes must either be strictly increasing or strictly decreasing, but the step size may vary. Also note that the lattice points are independent of those in the topography command.

The material surfaces should be given on the regular lattice

$$
d_{q, i, j}=\text { depth to surface number } q \text { at longitude } \phi_{i} \text {, latitude } \theta_{j} .
$$

The material surfaces correspond to material properties in the following way. At longitude $\phi_{i}$, latitude $\theta_{j}$ material number 1 (as defined by the material command) occupies depths $0 \leq d \leq d_{1, i, j}$. Material number 2 occupies depths $d_{1, i, j} \leq d \leq d_{2, i, j}$, and so on. If $d_{1, i, j}=0$, material number 1 is not used. Similarily, material number $k>1$ is not used if $d_{k-1, i, j}=d_{k, i, j}$. Material properties are only defined for depths down to the last surface, i.e.,

$$
0 \leq d \leq d_{N m a t, i, j}
$$

If the computational domain extends below the last material surface, it is necessary to use other commands to define the material properties in those regions.

Bi-linear interpolation in longitude and latitude is used to define the material surfaces in between the data points.

An example that uses an ifile material description is discussed in Section 9.3. 


\section{Chapter 6}

\section{Topography}

The topography command in WPP is used to specify the shape of the top surface of the computational domain,

$$
z=\tau(x, y)
$$

A curvilinear grid is automatically constructed between this surface and a user specified depth $z=\mathrm{zmax}$. If no topography command is present in the input file, the top surface is taken to be the plane $z=0$, and no curvilinear grid is constructed.

Three different topography descriptions are currently implemented in WPP: a Gaussian hill $(\S 6.1)$, a latitude-longitude grid file ( $(6.2)$, or topography from an Etree data base ( $\S 6.3)$.

\subsection{Gaussian hill topography}

The simplest type of topography is a Gaussian hill, which allows the user to place one Gaussian hill at a specified location in the $(x, y)$-plane. The user can adjust the amplitude of the hill as well as its spread in the $x$ and $y$-directions. This form of the topography command looks like this:

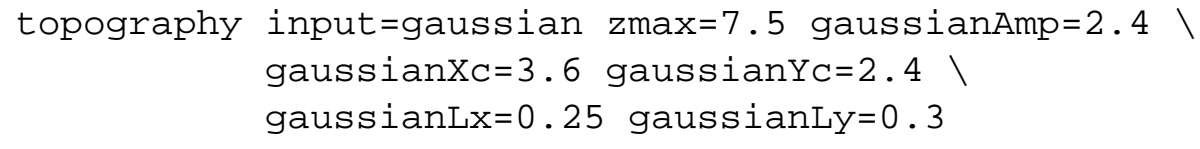

Note the zmax option, which tells $W P P$ to extend the curvilinear grid to $z=7.5$. The most common use of the Gaussian hill topography is for testing, see for example the input scripts in examples/twilight:

gauss-twi-1.in gauss-twi-2.in gauss-twi-3.in

\subsection{Topography grid file}

The topography can be given on a regular lattice in geographic (lat-lon) coordinates. This approach works well together with the block, pfile, and ifile material commands. When the material is described by an efile command, it is better to setup the topography from the same etree database, see Section 6.3.

To setup the topography for the Grenoble basin test case described in Section 9.3, you give the command topography input=grid file=grenobleCoarse.topo $\mathrm{zmax}=3000$ order $=2$

The file grenoblecoarse.topo holds the elevation (in meters) relative to mean sea level and must conform to the simple ASCII text format described in Section 11.3. In the above case, a curvilinear grid is constructed between the topography surface and $z=3000$, and the order $=2$ option specifies a second order polynomial stretching in the curvilinear mapping function. The topography is shown in Figure 6.1. 


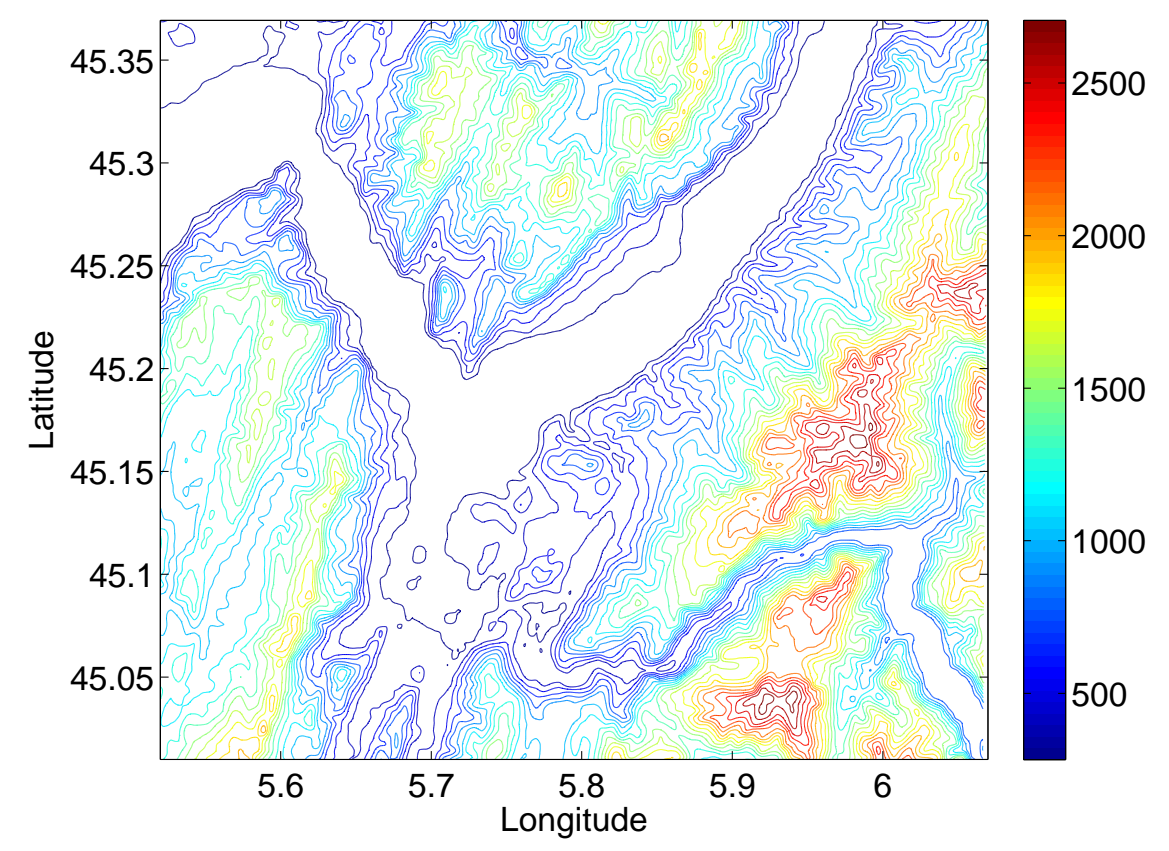

Figure 6.1: Topography in the vicinity of Grenoble, France.

\subsection{Etree topography}

The Etree data bases for the San Francisco bay area and Northern California contain topographic information. You can setup the computational grid to follow this topography by using the commands

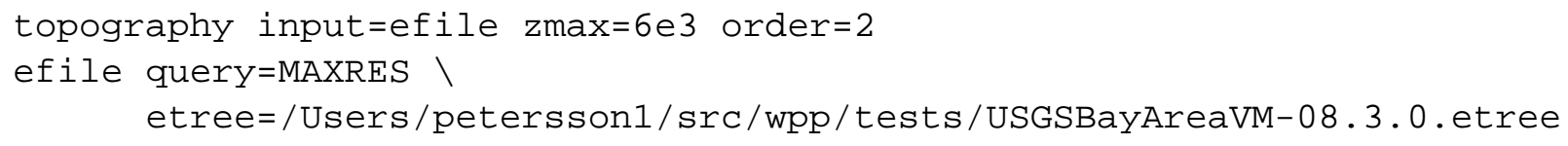

Here, the topography command tells WPP to read the topography from the Etree specified by the efile command. Hence, the topography command must be accompanied by an efile command. The order=2 option specifies the type of stretching to use when making the curvilinear grid. A higher value makes the curvilinear grid smoother near the bottom, but can cause a larger variation in grid size near the top. The zmax $=6 \mathrm{e} 3$ option tells $W P P$ to extend the curvilinear grid down to $z=6000$. As a rule of thumb, if the topography surface $z=\tau(x, y)$ varies between $\tau_{\min } \leq z \leq \tau_{\max }$ ( $z$ is positive downwards), you should use

$$
\mathrm{zmax} \geq \tau_{\max }+2\left(\tau_{\max }-\tau_{\min }\right),
$$

After reading the topography, WPP prints out the min and max $z$-coordinates. In the topography shown in Figure 6.2,

$$
\tau_{\min }=-1144.3, \quad \tau_{\max }=1092.9,
$$

which gives $\tau_{\max }+2\left(\tau_{\max }-\tau_{\min }\right)=5567.3$ Before the curvilinear grid is generated, the topography surface is smoothed by a Jacobi iteration. The purpose of the smoothing is to ensure that the variations in topography can be resolved on the computational grid. By default, 10 iterations are performed and this gives a satisfactory result in many cases. It is possible to change the number of iteration by using the smooth 


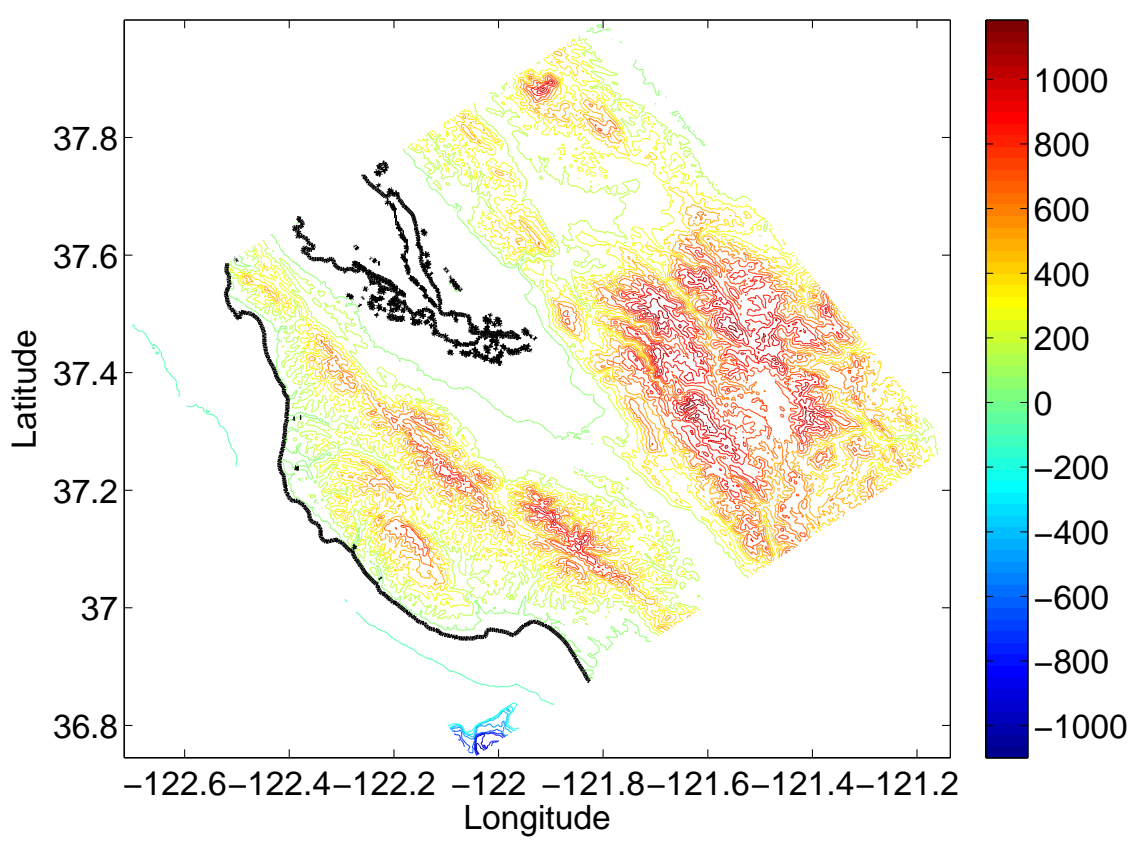

Figure 6.2: Topography and bathymetry in the vicinity of San Jose, south of San Francisco. The coastline is outlined by a thicker black line. Note the deep water in the Monterey Canyon, near the bottom corner of the computational domain.

option in the topography command. You can inspect the result of the smoothing by saving the top grid surface in an image file,

image mode=grid $z=0$ cycle=0 file=test

Note that the $z$ coordinate (positive downwards) is saved on a grid image file, while the elevation (positive upwards) of the raw topography is saved on a topo image file. 


\section{Chapter 7}

\section{Mesh refinement}

The refinement command in WPP enables the user to locally refine the computational mesh in areas where finer resolution is needed, i.e., where the wave speed is small. In order to maintain a constant resolution in terms of the number of grid points per wavelength for a given frequency (see Equation (4.1)), the grid size should be adjusted such that ratio $V_{s} / h$ becomes constant over the computational domain. However, the mesh size also needs to vary smoothly for the numerical solution to be accurate. In WPP, we compromise between these two requirements by using a composite grid approach consisting of a set of structured component grids with hanging nodes on the grid refinement interfaces. This allows the grid resolution to follow the main variations in wave speed, where each component grid has ideal wave propagation properties. An energy conserving coupling approach is used across grid refinement interfaces that guarentees stability of the numerical scheme, see [10] for details.

When using mesh refinement, the extent of the computational domain is determined by the grid command, which also specifies the grid size in the coarsest component grid,

grid $h=2000 \quad x=40000 \quad y=40000 \quad z=40000$

The two refinement commands

refinement $z \max =30000$

refinement $z \max =2000$

specify two mesh refinement interfaces: $z_{1}=30000$, and $z_{2}=2000$. As a result, the composite grid contains three component grids, where the coarsest component has grid size $h=2000$ and covers the bottom of the computational domain: $z_{1} \leq z \leq 40000$. Next refinement grid has half the grid size $(h=1000)$ and covers $z_{1} \leq z \leq z_{2}$. The grid size in the third component is another factor of two smaller $(h=500)$ and covers the top of the computational domain: $z_{2} \leq z \leq 0$. The composite grid is shown in Figure 7.1, where the grid is plotted in the vertical $x=20000$ plane. Note that refinement grids are aligned in the sense that every second grid point coincides with a grid point in the next coarser grid.

Mesh refinement can also be used together with topography. Here we use an example from the Alum Rock simulation described in 9.4. The composite grid is setup with the commands

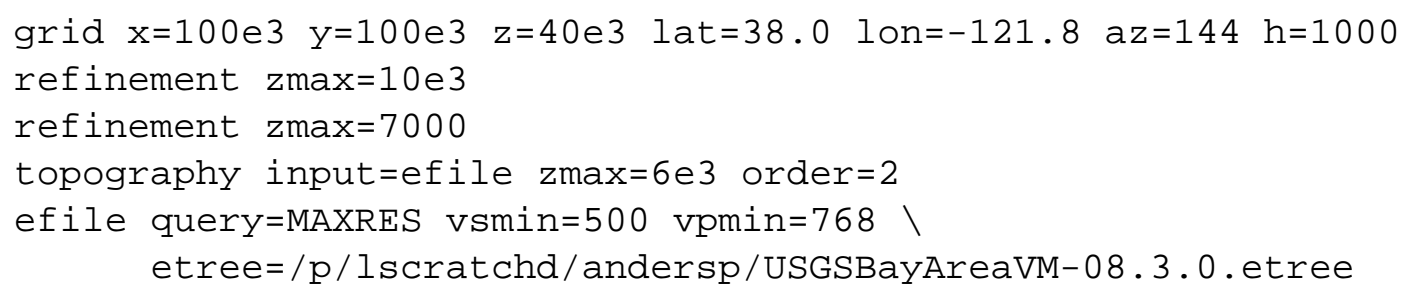




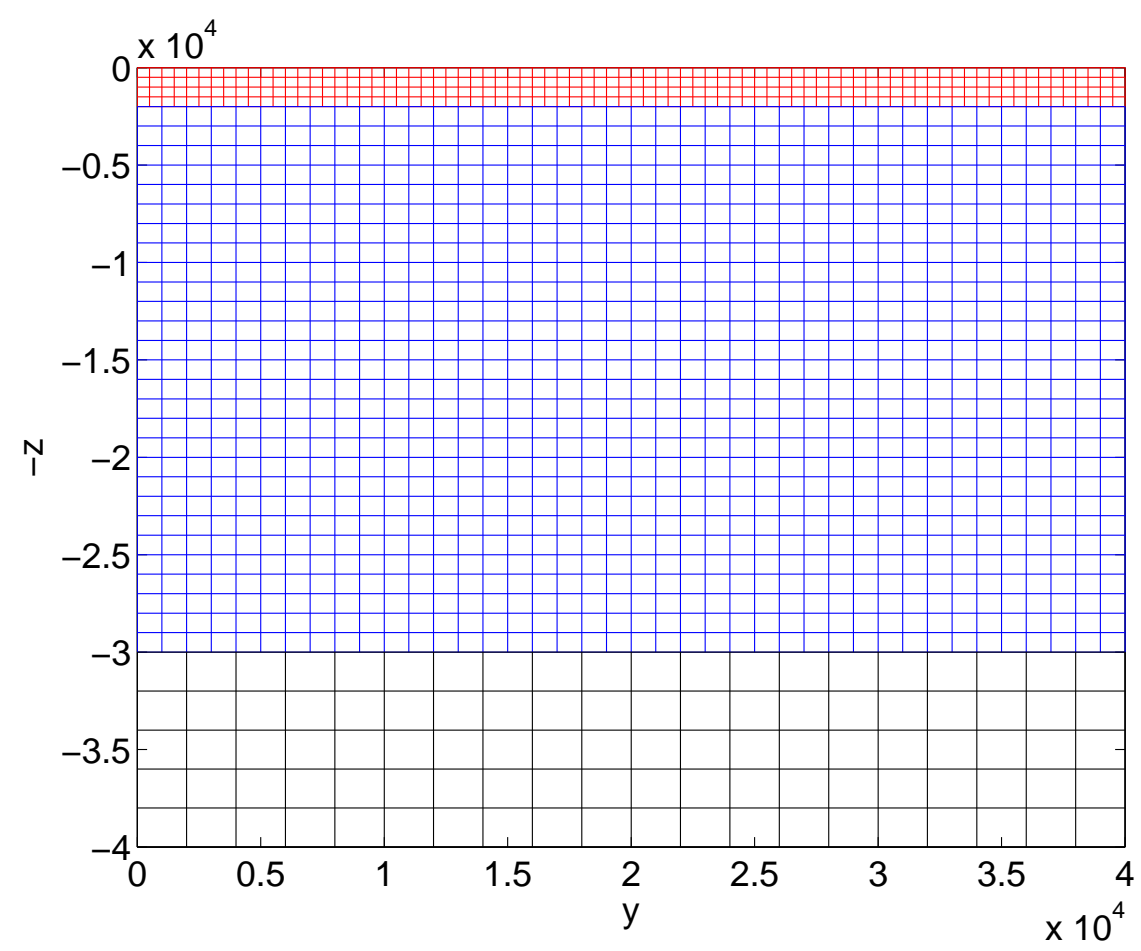

Figure 7.1: Composite grid with two mesh refinement interfaces and three Cartesian component grids.

Hence the coarsets Cartesian grid has grid size $h=1000$ and covers $10000 \leq z \leq 40000$. Next Cartesian grid has half the grid size $(h=500)$ and covers $7000 \leq z \leq 10000$. The grid size in the finest Cartesian component grid is reduced by another factor of two which gives $h=250$. This component extends to the bottom of the curvilinear grid, i.e., $7000 \leq z \leq 6000$. The vertical extent of the curvilinear grid is specified by the $z \max =6000$ option in the topography command, i.e., the curvilinear grid covers the domain between $z=6000$ and the topography, $z=\tau(x, y)$. In the horizontal directions, the grid size in the curvilinear component is the same as the finest Cartesian component. The number of grid points in the vertical direction is choosen such that the average vertical grid size is the same as the grid size in the horizontal directions. A portion of the computational grid is shown in the vertical cross section $x=50000$, see Figure 7.2.

The image command saves the solution on the base grid and on all refinement grids that intersect a given image plane. 


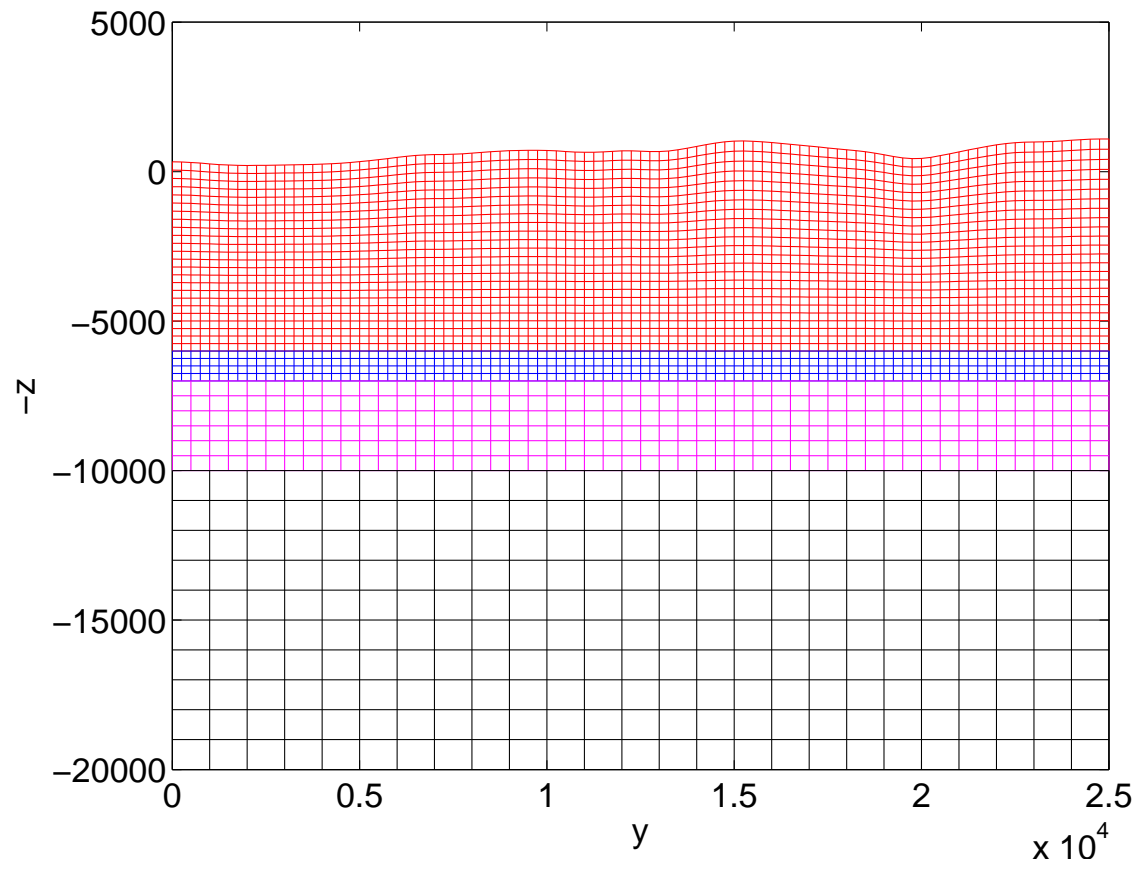

Figure 7.2: A composite grid with two mesh refinement interfaces and topography. In this case there are three Cartesian components and one curvilinear grid following a non-planar topography. 


\section{Chapter 8}

\section{Output options}

\subsection{Setting the output directory}

The fileio command can be used to specify a directory where WPP writes its output files. If the directory does not exist, WPP attempts to create it for you. The fileio command may also be used to set the level of diagnostic messages (verbose) and how often the time step information is printed. For example,

fileio path=wpp_dir verbose=1 printcycle=10

causes all output files to be written to the directory "./wpp_dir", turns on some extra diagnostic messages (a higher value gives more details), and prints the time step information every 10 time steps.

Serial and Parallel file systems Some parallel machines have a dedicated parallel file system which allows many processors to simulataneously write to the same file. These file systems are often mounted on a special directory. By default, WPP assumes a serial file system and will only let one processor write to the same file at the same time. If you have access to a parallel file system, the I/O performance of WPP can sometimes be improved by allowing several processors to simultaneously write a file. You enable this feature by using the $\mathrm{p} f \mathrm{~s}=1$ option,

fileio pfs=1 path=/p/lscratcha/my_output_directory

Note that many parallel file systems are only accessible from dedicated directories. Enabling pf $s=1$ without re-directing the output to a parallel file system can cause WPP to either crash or hang.

\subsection{Time-history at a reciever station: the sac command}

WPP can save the time-history of the solution at a receiver station located anywhere in the computational domain. The basic command looks like this:

sac $x=100 e 3 \quad y=50 e 3 \quad z=0$ file=sta 1

Here, WPP saves the three components of the solution at the grid point that is closest to the specified $(x, y, z)$ location. By default, WPP saves the data using the binary Seismic Analysis Code (SAC) [4] format. In the above case, the sac command results in three files:

stal.x stal.y stal.z 
The $\mathrm{x}, \mathrm{y}, \mathrm{z}$ files hold the solution time-history in the corresponding coordinate direction. Note that a positive $z$-component corresponds to a downward motion.

The location of the reciever station can alternatively be given in geographic (latitude, longitude) coordinates. Information about the event date, time, and station name is saved in the header of a SAC file. By default the date and time are set to the date and time at the start of the simulation; the default station name is the name of the file. The default values of these fields can be changed by using the eventData, event Time, and sta options,

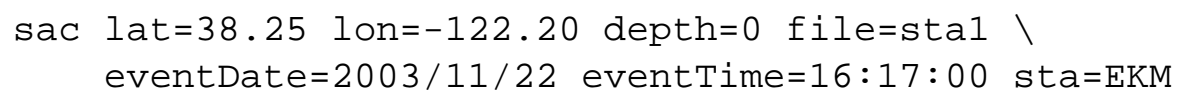

Note that depth specifies the depth of the reciever below the topography. To place a reciever at elevation $e$ relative to mean sea level ( $e$ is negative below sea level) you can replace the depth option by $z=-e$.

By default, SAC files are written to disk every 1000 time steps, and at the end of the simulation. We can change this frequency by using the writeEvery option. For example, to write the SAC file every 100 time steps, you would say

sac lat $=38.25$ lon=-122.20 depth=0 file=stal writeEvery=100

By default, WPP outputs the three components of the solution $\mathbf{u}\left(\mathbf{x}_{r}, t\right)=\left(u_{x}, u_{y}, u_{z}\right)^{T}$. By using the velocity=1 option, WPP instead outputs the three components of the time-derivative of the solution, i.e., the velocity if $W P P$ is setup to solve for displacements. The nsew=1 option can also be used to tell WPP to rotate the solution components to the East, North, and vertical (positive up) directions,

sac lat $=38.25$ lon=-122.20 depth=0 file=stal velocity=1 nsew=1

The angle between North and the $x$-axis is determined by the azimuth $(\mathrm{a} z=\ldots)$ angle in the grid command:

grid $x=100 e 3 \quad y=50 e 3 \quad z=30 e 3$ lat $=37.5$ lon=-122.0 az=135

To remind the user of what quantities are saved in a sac file, we modify the file name extensions according to the following table:

\begin{tabular}{|c|c|c|}
\hline & velocity=0 & velocity=1 \\
\hline nsew=0 & $. x, . y, . z$ & $. x v, . y v, . z v$ \\
\hline nsew=1 & $. e, . n, . u$ & $. e v, . n v, . u v$ \\
\hline
\end{tabular}

$W P P$ can also output reciever time-histories in an ASCII text format,

sac lat $=38.25$ lon=-122.20 depth=0 file=stal sacformat=0 usgsformat=1

The ASCII text file holds all three components in a single file named sta1 . $t x t$. When the usgs format=1 option is used, the file gets extension .txt independently of the nsew and velocity options. Instead the header inside the file is modified to reflect its contents. Note that you must give the sacformat $=0$ option unless you want the solution to be output in both formats.

\section{Notes on the sac command:}

- SAC files are treated in the same way on parallel and serial file systems, because the data for each SAC file originates from one processor and is always written by that processor only.

- The binary SAC format is described in Section 11.4. 
- The ASCII text format is outlined in the header of those files.

- The binary SAC files can be read by the SAC program. We also provide a matlab/octave script in tools/readsac.m.

- The ASCII text file format can be read by the matlab/octave script in tools/readusgs.m.

\subsection{2-D cross-sectional data: the image command}

The image command saves two-dimensional horizontal or vertical cross-sectional data at a specified time level. It can be used for visualizing the solution, making the images for a movie, or checking material properties. Each image file contains a scalar field as function of the spatial coordinates in the cross-sectional plane. The scalar field can be either a component of the solution, a derived quantity of the solution, a material property, or a grid coordinate, All in all, WPP can output twenty-five different types of images, see Section 10.4.2 for details.

The cross-sectional plane is specified by a Cartesian coordinate $(x, y$, or $z)$. The image can be written at a specific time step or at a specified time. Images can also be output at a fixed frequency, either specified by a time step interval or a time interval.

For example, the command

image mode=ux y=500 file=picturefile cycle=1

tells WPP to output the $x$-displacement component of the solution along the vertical $y=500$ plane. The data is written to a file named picturefile. cycle=1.y=500. ux after the first time step (cycle=1). The example

image mode=div $\mathrm{x}=1000$ file=picturefile cycleInterval=100

outputs the divergence of the solution field in the $y z$-plane at the grid surface closest to $x=1000$. The data is written to the files

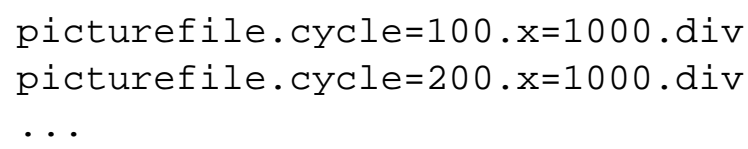

With this setup, one image file is output every 100 time steps.

Note that the divergence of the solution field does not contain shear (S) waves and the rotation (curl) of the solution field does not contain compressional (P) waves. These options can therefore be used to distinguish between $\mathrm{P}$ - and $\mathrm{S}$-waves in the solution.

The hvelmax and vvelmax modes store the maximum in time of the horizontal and vertical velocity components, respectively. As these names indicate, it is assumed that the sources in WPP are set up for calculating displacements. The horizontal velocity is defined as $\max \left(\left|u_{t}^{N}\right|,\left|u_{t}^{E}\right|\right)$, where $u^{N}$ and $u^{E}$ are the displacement components in the North and East directions, respectively. The vertical velocity is $\left|w_{t}\right|$, where $w$ is the displacement component in the $z$-direction. For these modes, the cycleInterval or timeInterval options only determine how often the maxima are written to disk; the actual accumulation of the maximuma is performed after each time step.

When WPP is run in parallel, the data that gets saved on an image file originates from all processors that are intersected by the image plane. For horizontal image planes, this means all processors. To improve the $\mathrm{I} / \mathrm{O}$ performance, image data is first communicated to a number of dedicated image writing processors. By default, 8 processors write each image file to disk (or all processors if WPP is run on fewer than 8). This number can be changed using the fileio command, 


\section{fileio nwriters $=4$}

The above command tells WPP to use 4 processors to write each image file. For simulations which use very large number of grid points and many processors, care must be taken to make sure that enough memory is available to buffer the image data before it is written to disk.

\section{Notes on the image command:}

- By default, single precision data is saved. Double precision data can be saved by using the precision=double option.

- When topography is used, an image plane along the free surface is specified by the $z=0$ option.

- A mode=topo $z=0$ image holds the elevation (negative $z$-coordinate) of the raw topography. It can only be written when topography is used.

- A mode=grid $z=0$ image holds the $z$-coordinate (negative elevation) of the grid along the free surface, which is the actual shape of the upper surface of the computational domain.

- When topography or mesh refinement is used, vertical image planes intersect all component grids in the composite grid. In this case, cross-sectional data from all component grids are stored on the image file.

- The images files are written in a binary format, see Section 11.5 for details.

- We provide matlab/octave scripts for reading image files in the tools directory. The basic function is called readimagepatch.m. A higher level interface is provided by the imageinfo.m and plotimage.m scripts.

\subsection{Generating a bird's eye view of the problem domain: the gmt command}

The Generic Mapping Toolkit (GMT) [11] is a set of postscript image generation programs for geophysical applications, which can be used to make plots like Figure 8.1. In the example shown here, topography information is included as well as information on the general setup of the simulation. Note that the file which is output from WPP is a UNIX C-shell script, which often needs to be fine-tuned to suit the needs of a particular application. To have WPP write a GMT file, you give the command

gmt file=bolinas.gmt 


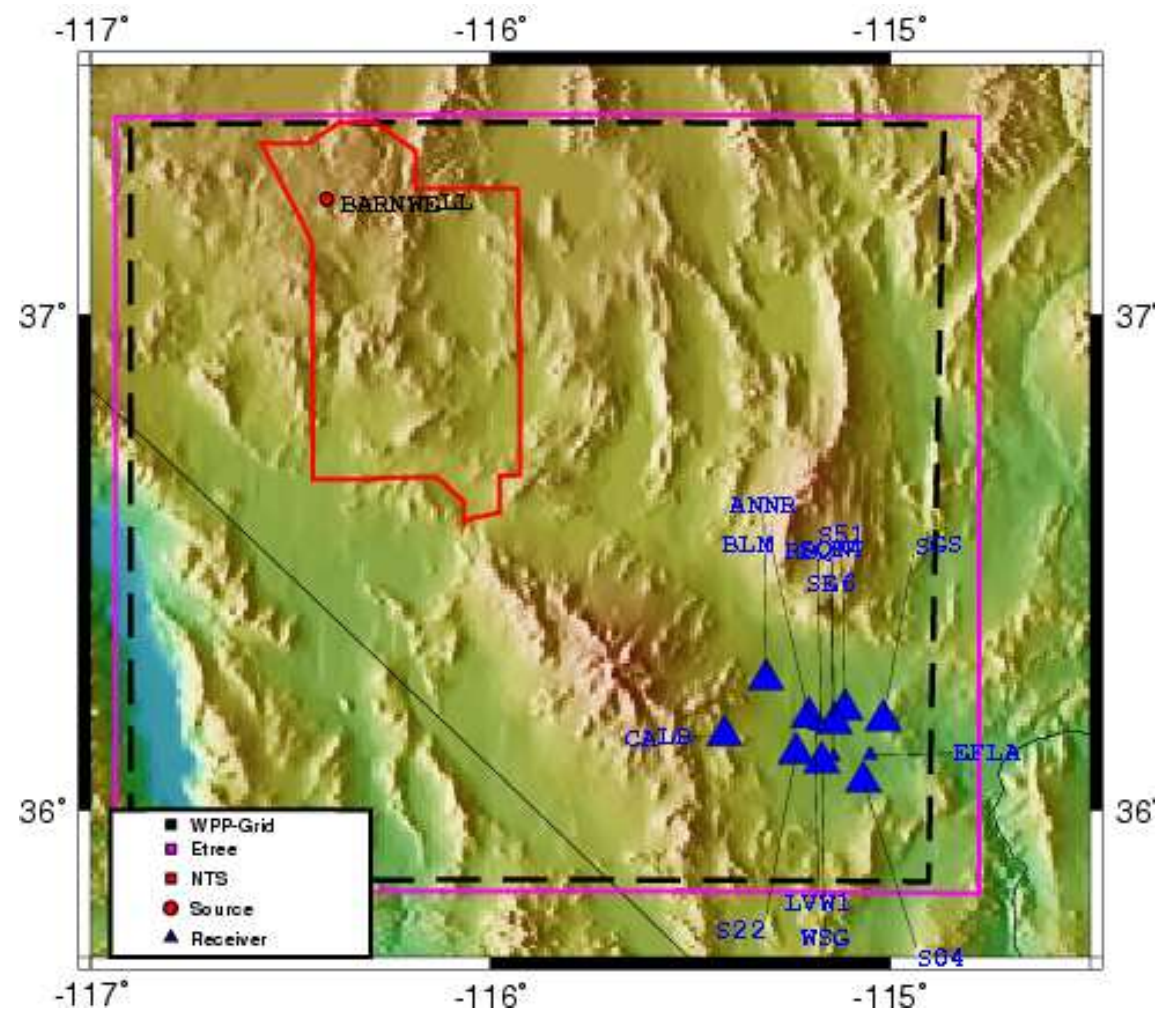

Figure 8.1: Location of the source and stations for the Barnwell simulation. This figure was generated using the GMT command, see Section 10.4.3 for details. 


\section{Chapter 9}

\section{Examples}

This chapter describes most of the the input scripts in the directory examples. The output associated with input file $x y z$. in is given in $x y z$. out.

\subsection{Lamb's problem}

The version of Lamb's problem [6] considered here consists of a single vertical time-dependent point force acting downward on the surface of a homogeneous half-space. In this section, we use the analytical solution from Mooney [8] to test the accuracy of the numerical solution.

In the following example, the elastic half-space consists of a Poisson solid $(\lambda=\mu)$ with $\mathrm{S}$-wave velocity $V_{s}=1000 \mathrm{~m} / \mathrm{s}$, P-wave velocity $V_{p}=1000 \cdot \sqrt{3} \mathrm{~m} / \mathrm{s}$, and density $\rho=1500 \mathrm{~kg} / \mathrm{m}^{3}$. The elastic half-space is truncated to the computational domain

$$
(x, y, z) \in[0,8000] \times[0,8000] \times[0,4000] .
$$

The source is placed on the free surface in the center point of the horizontal plane: $(4000,4000,0)$. The time dependency of the forcing is a "RickerInt" (see Figure 4.2) with $\omega=1 \mathrm{~Hz}, t_{0}=2 \mathrm{~s}$ and magnitude $10^{13} \mathrm{~N}$. The above setup is created with the input file shown below, which can be found in examples/lambtests/seismicl.in

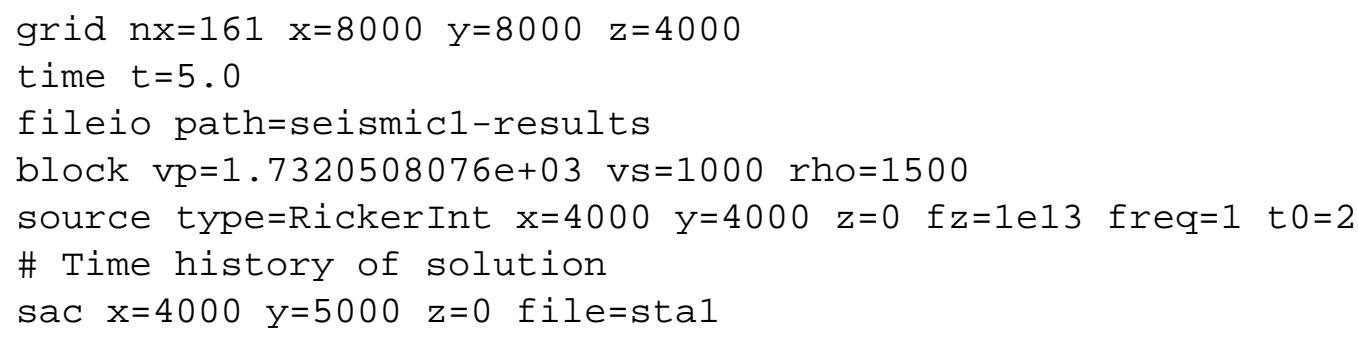

The vertical displacement at the reciever $(x=4000, y=5000, z=0)$ and the error for three grid sizes can be found in Figure 9.1.

The waveforms are all smooth and the problem appears to be well resolved. We present the max-norm of the errors in the vertical displacement in Table 9.1. The ratio between errors as the grid size is halved approaches 4 for the finest grid, indicating that the numerical method and the discretization of the point force are second order accurate. The center frequency in the RickerInt time function is $f_{0}=1 \mathrm{~Hz}$. Following (4.3), we estimate the highest significant frequency to be $f_{\max }=2.5 \mathrm{~Hz}$. In this case the formula for the number of grid points per wave length (4.1) becomes $P=1000 /(2.5 h)$. Note that $P=16$ gives a relative max-norm error of about 3.5 percent. 


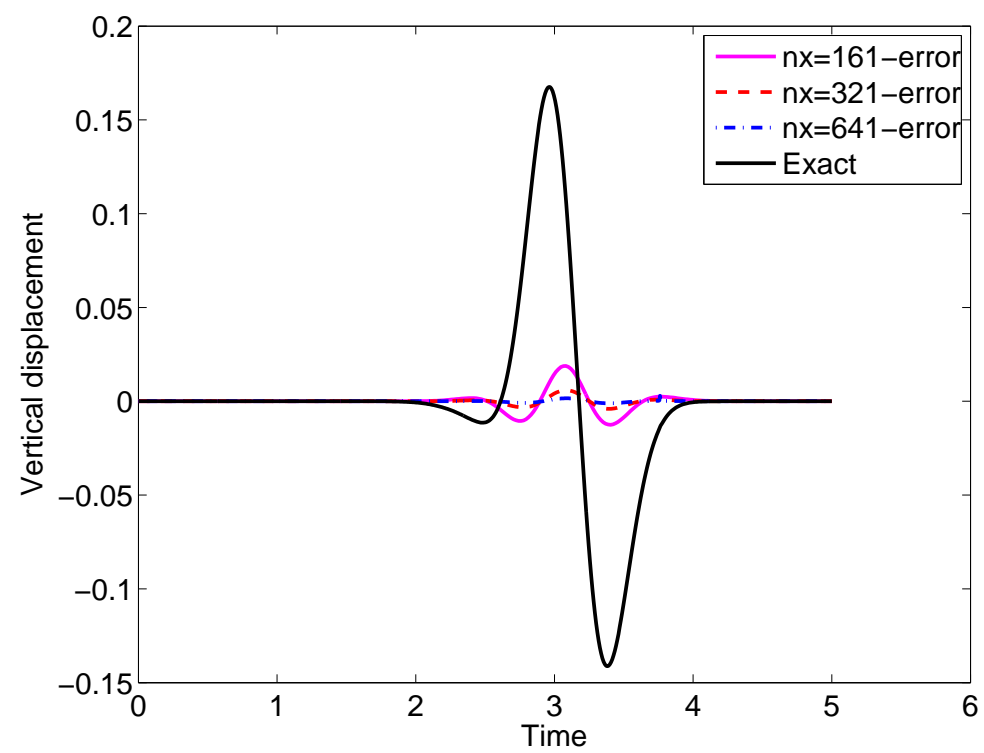

Figure 9.1: Lamb's problem: Vertical displacement at reciever $x=4000, y=5000, z=0$. The black line shows the exact solution, while the magenta, red and blue lines show the errors in the numerical solutions with different grid resolution.

\begin{tabular}{|c|c|c|c|c|}
\hline$N_{x}$ & $h$ & $P$ & $\left\|u_{z}-U_{z}\right\|_{\infty} /\left\|U_{z}\right\|_{\infty}$ & ratio \\
\hline 161 & 50 & 8 & $1.12 \cdot 10^{-1}$ & - \\
\hline 321 & 25 & 16 & $3.54 \cdot 10^{-2}$ & 3.16 \\
\hline 641 & 12.5 & 32 & $9.47 \cdot 10^{-3}$ & 3.74 \\
\hline
\end{tabular}

Table 9.1: Max norm errors in the vertical displacement at reciver $x=4000, y=5000, z=0$.

\subsection{Examples from Lifelines project 1A01: Validation of basin response codes}

The following examples are taken form the Lifelines project 1A01: Validation of basin response codes, see [3]. A detailed description of the setup of the Layer over halfspace (LOH) problems can also be found in [3]. To enable a direct comparison with those results, the WPP simulations are set up to calculate velocities as opposed to displacements.

\subsubsection{The LOH.1 problem}

The LOH.1 problem, defined in the input script examples/scec/LOH.1.h50.mr.in, has a layered material model where the top 1000 meters $(z \in[0,1000 m])$ has different properties than the rest of the domain. The computational domain is taken to be $(x, y, z) \in[0,30000]^{2} \times[0,17000]$. The grid size in the base (coarsest) grid is choosen to be $h=50 \mathrm{~m}$ and the material properties in the different layers are described by

grid $h=50 \quad x=30000 \quad y=30000 \quad z=17000$ extrapolate $=2$ 
block vp $=4000$ vs $=2000$ rho $=2600$

block $v p=6000$ vs=3464 rho=2700 z1=1000

The problem is forced by a single point moment source, positioned in the lower half-space. The time function in this problem is a Gaussian (if setup as in the input file, the Gaussian source is equivalent to using a Brune time function followed by a post processing deconvolution step, as is described in [3]). The advantage of using the Gaussian is that no post processing is necessary before comparing to the results in [3], and the Gaussian function produces less high wave number waves which are poorly resolved on the computational mesh. Note that $\mathrm{freq}=16.6667$ corresponds to the spread $\sigma=0.06$ in the Gaussian time function. The lines to setup the source and the time duration of the simulation are:

time $t=9$

source $\mathrm{x}=15000 \mathrm{y}=15000 \mathrm{z}=2000 \mathrm{Mxy}=1 \mathrm{m0}=1 \mathrm{e}=18$ t0=0.36 freq=16.6667।

type=Gaussian

The layered velocity structure makes this problem an ideal candidate for mesh refinement. We align the refinement level with the material discontinuity by specifying

refinement $z \max =1000$

As a result the grid size in the top 1000 meters $(0 \leq z \leq 1000)$ will be $h=25$ meters. The extrapolate $=2$ option in the above grid command tells WPP to extrapolate material properties to the ghost point and the point on the interface. This gives uniform material properties on each component grid and allows the jump conditions across the material discontinuity to be handled accurately. The extrapolate option should only be used when the grid interface is perfectly aligned with the material discontinuity.

The solution is recorded in an array of receivers:

$\begin{array}{lllll}\operatorname{sac} x=15600 & y=15800 & z=0 & \text { file=sac_01 } \\ \text { sac } x=16200 & y=16600 & z=0 & \text { file=sac_02 } \\ \text { sac } x=16800 & y=17400 & z=0 & \text { file=sac_03 } \\ \text { sac } x=17400 & y=18200 & z=0 & \text { file=sac_04 } \\ \text { sac } x=18000 & y=19000 & z=0 & \text { file=sac_05 } \\ \text { sac } x=18600 & y=19800 & z=0 & \text { file=sac_06 } \\ \text { sac } x=19200 & y=20600 & z=0 & \text { file=sac_07 } \\ \text { sac } x=19800 & y=21400 & z=0 & \text { file=sac_08 } \\ \text { sac } x=20400 & y=22200 & z=0 & \text { file=sac_09 } \\ \text { sac } x=21000 & y=23000 & z=0 & \text { file=sac_10 }\end{array}$

Numerical velocity time histories for station 10 are shown in Figure 9.2 together with a semi-analytical solution. We conclude that most features in the solution are very well captured on this grid. As is customary in seismology, the velocity components have been rotated to polar componenets, with the origin at the source. The sac command outputs the $u_{x}, u_{y}$ and $u_{z}$-components of the velocity. These components are rotated to radial and transverse components using the transformation,

$$
u_{\text {rad }}=0.6 u_{x}+0.8 u_{y}, \quad u_{\text {tran }}=-0.8 u_{x}+0.6 u_{y} .
$$

The vertical component is given by $u_{z}$ (positive downwards).

By using formulas (4.1)-(4.3), we can calculate the number of points per wave length for this simulation. Since we are using a Gaussian time-function, the center frequency is $f_{0}=1 /(2 \pi \sigma) \approx 2.6526$ and we 

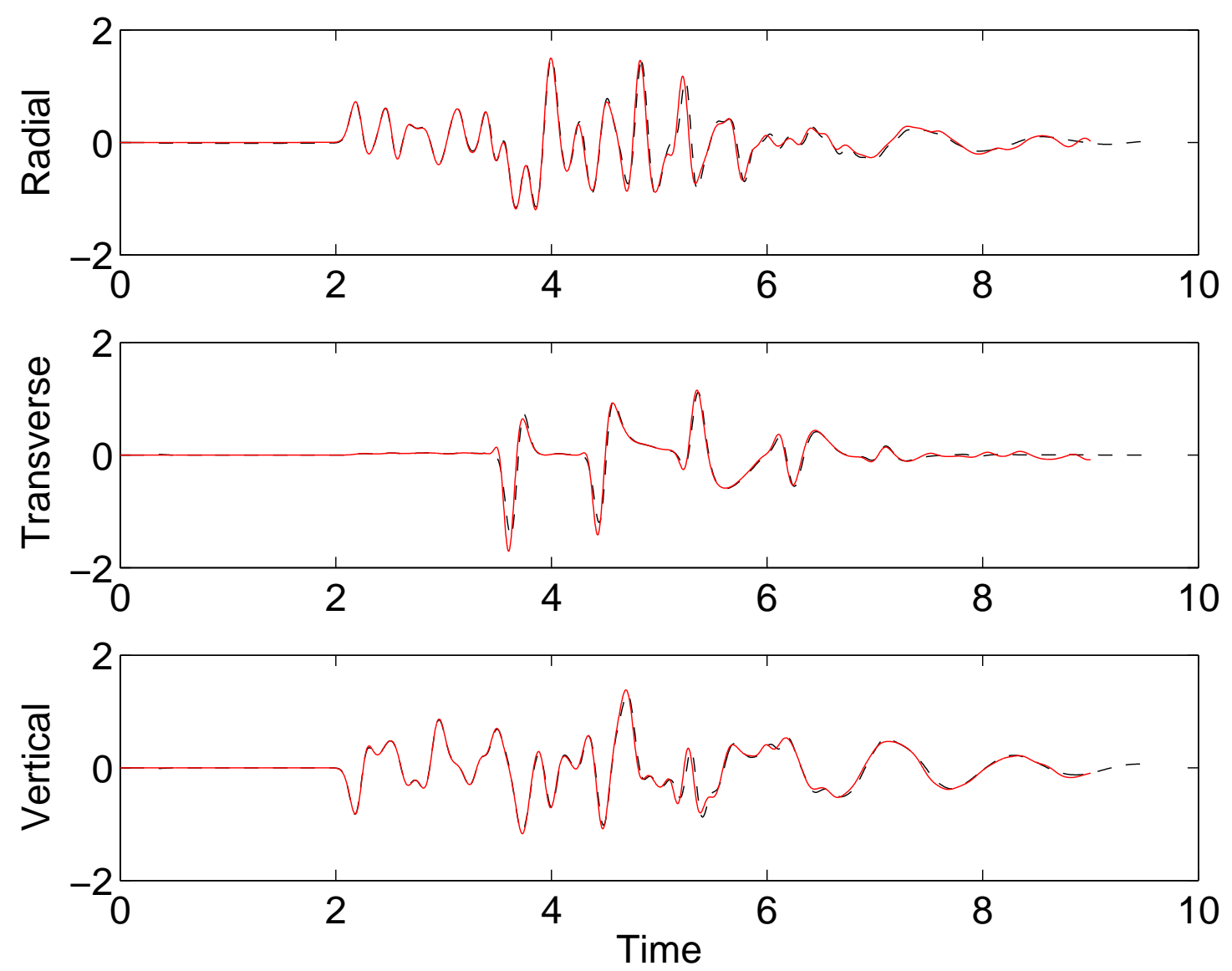

Figure 9.2: LOH.1: The radial (top), transverse (middle) and vertical (bottom) velocities for receiver number 10. Here the numerical solution is plotted in red while the semi-analytical solution is represented by dashed black lines.

estimate the upper power frequency to be $f_{\max } \approx 2.5 f_{0}=6.6315 \mathrm{~Hz}$. The material model has $\min V_{s}=$ $2000 \mathrm{~m} / \mathrm{s}$ where the grid size is $h=25 \mathrm{~m}$, and we arrive at

$$
P=\frac{2000}{25 \cdot 6.6315} \approx 12.1
$$

From our discussion in Section 4.3, 12.1 points per wave length is on the low side, but visual inspection of Figure 9.2 indicates very good agreement of the wave forms.

\subsubsection{The LOH.2 problem}

The geometrical setup of the LOH.2 problem is identical to that of LOH.1, but the LOH.2 problem models an earthquake along a fault plane. The slip along the fault is modeled by a large number of point moment tensor sources with the time dependency given by the Gaussian function with different offsets in time (depending on distance from the hypocenter). The fault plane coincides with the y-z-plane in the computational grid. The input files for LOH.2 can be found in examples/scec/LOH.2.h50.mr. in.

As for LOH.1, a semi-analytical solution is available for $\sigma=0.06$ corresponding to the frequency parameter freq $=16.6667$ in all source commands. This leads to the same number of grid points per 
wave length as for LOH.1.

$$
P \approx 12.1
$$

In Figure 9.3, we evaluate the error in the solution at station 10, by comparing velocitiy time-histories in the numerical solution to a semi-analytical solution. We conclude that most features in the solution indeed are captured on this grid, in particular before time $t \approx 5.5$. At later times, artificial effects of the outflow boundary dominate the solution error. These effects are larger than in LOH.1 because the sources are distributed in space so some sources are closer to the outflow boundary than in LOH.1.
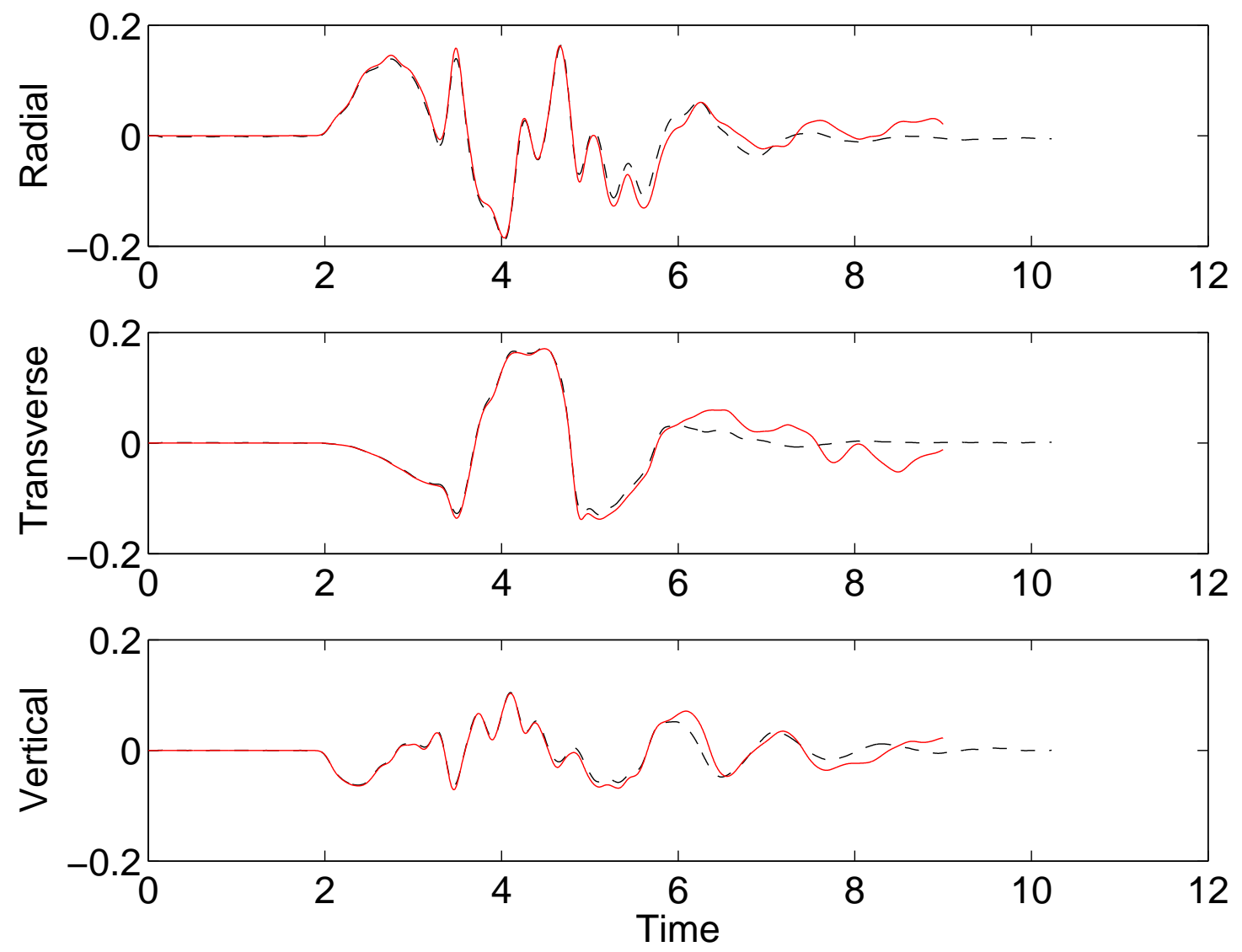

Figure 9.3: LOH.2: The radial (top), transverse (middle) and vertical (bottom) velocity components recorded at station number 10. Here the dashed black line is a semi-analytical solution and the red line is the numerical solution.

\subsection{The Grenoble basin test case}

This example uses realistic topography and mesh refinement to model a scenario earthquake near Grenoble, France. The WPP input file for this simulation is called Grenoble. in and can be found in the examples/ifile directory.

Grenoble is located in a Y-shaped valley in the foothills of the Alps. The extent and geographic orientation of the computational domain is described by

grid $\mathrm{x}=40 \mathrm{e} 3 \mathrm{y}=43 \mathrm{e} 3 \mathrm{z}=40 \mathrm{e} 3$ lon $=5.52$ lat $=45.01$ az=0 $\mathrm{h}=200$ 
Hence, the $x$-axis points in the direction of North $(\mathrm{az}=0)$ and the $y$-axis is directed due East. With this orientation, the lon and lat options specify the location of the South-West corner of the computational domain.

To setup the topography we give the command

topography input=grid file=grenoblecoarse.topo zmax=3000 order=2

The file grenobleCoarse.topo holds the elevation (in meters) above mean sea level on a regular grid in geographic (lat-lon) coordinates. A plot of the topography can be found in Figure 6.1. The material properties are described by a heterogeneous model with granite and sediment. The properties of the granite are assumed to only depend on depth, and are setup using block commands,

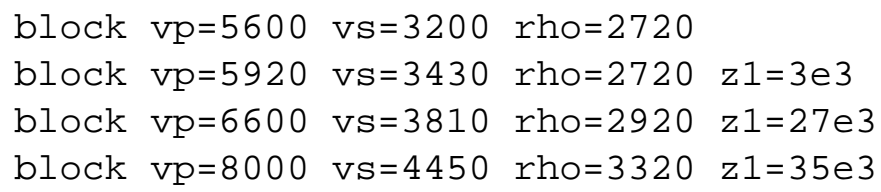

Since the material commands are read in the order they occur, the properties of the top 3000 meters are described by the first block command, and the subsequent block commands describe the properties deeper into the earth because their extent is restricted by the $\mathrm{z} 1$ option (note that $\mathrm{z} 1$ and $\mathrm{z} 2$ correspond to depth in the presence of topography).

The geometry of the sedimentary basin is described by the ifile command,

ifile filename=bedrock_surface.dat

In this case, the file bedrock_surface.dat holds the depth of the sedimentary basin on a regular grid in geographic (lon-lat) coordinates. Note that this grid is unrelated to the computational grid and the grid used in the topography file. The format of this ASCII text file is described in Section 11.3. The ifile command can be used to describe the depths of several material surfaces, but in this case we only have one.

For each material surface in the ifile command, there must be a material command with a unique id number. The material properties between the free surface and the first material surface in the ifile are defind by the material command with the lowest id number, and so on. The ifile command only assigns material properties down to the depth of the last material surface.

In our case, the material properties of the sediment, i.e., between the free surface and the single material surface in the ifile command are described by

material id=1 vs=300 vp=1450 vpgrad=1.2 rho=2140 rhograd=0.125

Note that the depth of the material surface is relative to the topography, which means that no sediment is present where the depth is zero. By plotting the compressional wave speed along the top surface and in a vertical cross-section, we can see both the horizontal extent and the variable depth of the sedimentary basin, see Figure 9.4.

The slowest shear speed in the model is $300 \mathrm{~m} / \mathrm{s}$ (in the sediment) and the fastest compressional speed is $8,000 \mathrm{~m} / \mathrm{s}$ (in the granite deeper than $35 \mathrm{~km}$ ). To reduce the ratio between the shortest and longest wave in the solution, we can impose a minimum threshold on the material velocities through the command

globalmaterial vpmin=800 vsmin=500

The slow material in the sedimentary basin sets the grid size requirement for the simulation. To reduce the total number of grid points, we use local mesh refinement to coarsen out the computational grid below the curvilinear grid (that extends to $\mathrm{zmax}=3000$ ), 

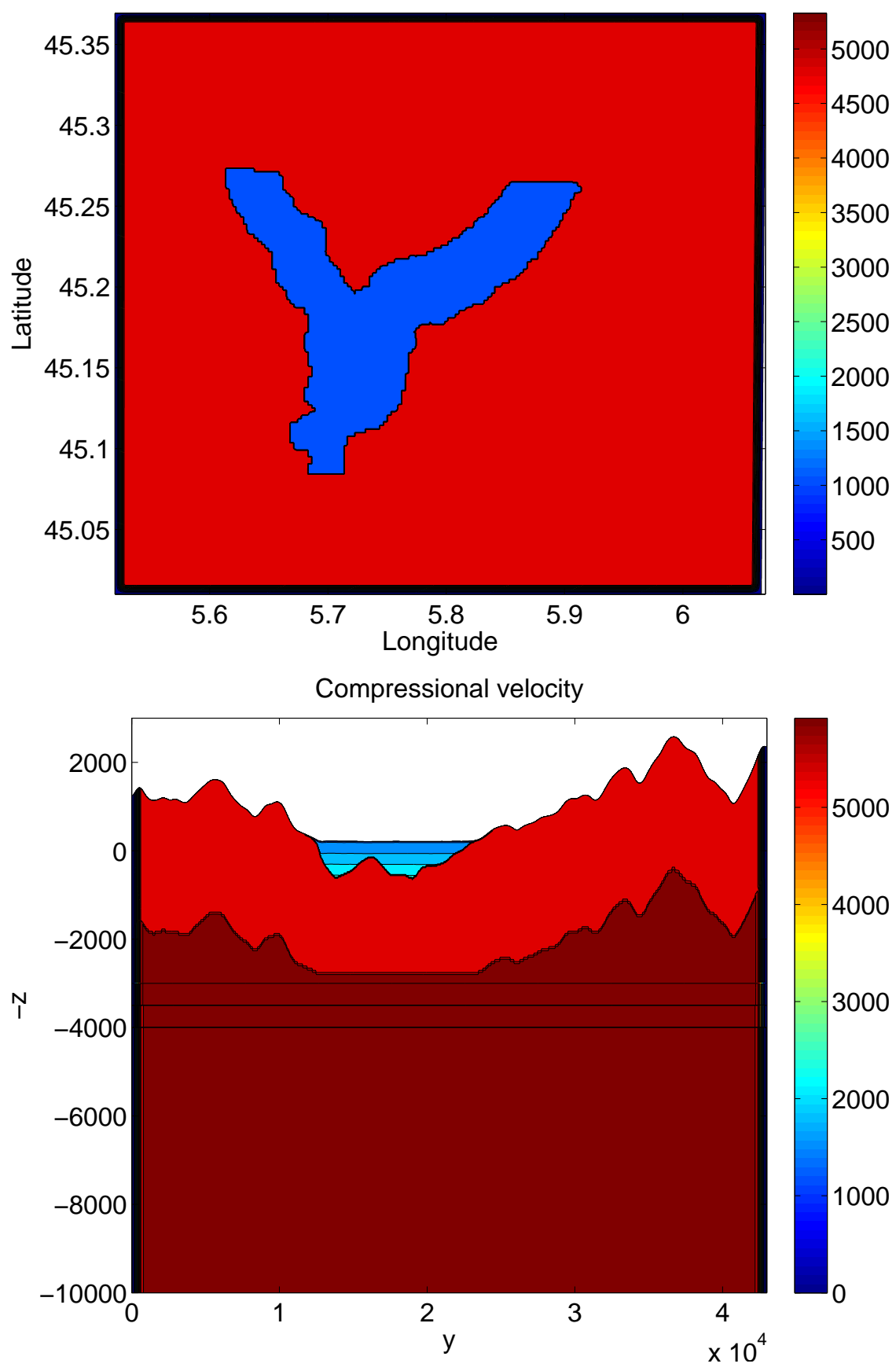

Figure 9.4: Grenoble basin model. Top: compressional velocity along the free surface, illustrating the extent of the sedimentary basin. Bottom: compressional velocity in the vertical cross-section $x=20,000$ (latitude $\approx 45.2^{\circ}$ ). The black horizontal lines mark the bottom of the curvilinear grid and the mesh refinement interfaces for the Cartesian component grids. 
refinement $z \max =3500$

refinement $z \max =4000$

Since the base grid has grid size $h=200 \mathrm{~m}$, the two refined Cartesian grids get grid sizes $h=100 \mathrm{~m}$ and $h=50 \mathrm{~m}$, respectively. The average grid size in the curvilinear grid, which covers the sedimentary basin, is therefore $h=50 \mathrm{~m}$.

The source mechanism in this small $\left(M_{w}=2.9\right)$ scenario earthquake corresponds to case $\mathrm{W} 1$ in the ESG2006 benchmark,

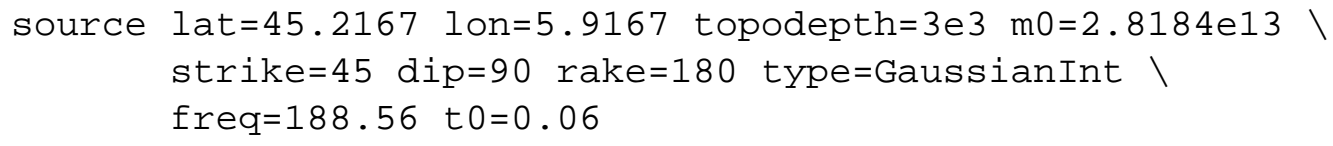

Note the very high angular frequency in the GaussianInt time function, corresponding to a center frequency of $f_{0} \approx 30.0 \mathrm{~Hz}$. The corresponding upper power frequency would be $f_{\max } \approx 75 \mathrm{~Hz}$. With a minimum shear speed threshold of $500 \mathrm{~m} / \mathrm{s}$, this corresponds to a smallest wave length of $L_{\min }=500 / 75 \approx 6.67$ $\mathrm{m}$, making a fully resolved simulation extremely challenging. Instead of artificially lowering the freq parameter, we choose to filter the source time function with a two-pass two-pole Butterworth filter with corner frequency $1 \mathrm{~Hz}$,

prefilter $\mathrm{fC}_{\mathrm{C}}=1.0$

The resulting motion is identical to running the simulation with the original time function followed by time filtering the motion at all points in space using the same filter.

If we use this corner frequency to estimate the shortest wave length, we get $L_{\min } \approx 500 \mathrm{~m}$, and the curvilinear grid size $h=50$ should provide acceptable resolution with $P=10$ grid points per wave length.

In order to avoid incompatibilities due to the exponentially decaying tails in the filtered source time function, WPP automatically adjusts the starting time of the simulation from $t_{0}=0$ to $t_{0} \approx-3.97$. As a result the requested 15 seconds of simulation time corresponds to final time $t_{0}+15 \approx 11.03$. The calculated displacement time history at one station as well as the peak horizontal velocities along the free surface are shown in Figures 9.5-9.6.

The input file Grenoble. in is setup to report the motion at many other stations, and to save several different image files of the material model and the solution. We encourage the reader to run this case for themselves, and explore the results further.

\subsection{Modeling the October 2007, Alum Rock earthquake}

This example uses both realistic topography and mesh refinement. The material properties and topography are obtained from an Etree data base developed by the USGS. Hence, before you can run this case, you must download the data base from the USGS website, see Section 5.2. It is also necessary to configure WPP to use the efile command, see Section A.5.

The input scripts are located in the directory wpp-version-2.0/examples/efile:

\section{Alumrock.in Alumrock-2.in}

The main difference between these input files is the grid size. The case in the Alumrock . in file uses only about 729,000 grid points and can easily be run on a workstation. The grid size in the top grid is $h=500$ $\mathrm{m}$, so this simulation can only be expect to capture very long period motions (frequencies up to $0.1 \mathrm{~Hz}$ ). The Alumrock-2. in case uses half the grid size, leading to about 5.4 Million grid points and captures frequencies up to $0.2 \mathrm{~Hz}$. This case can also be run on a workstation as long as it has enough memory, but 


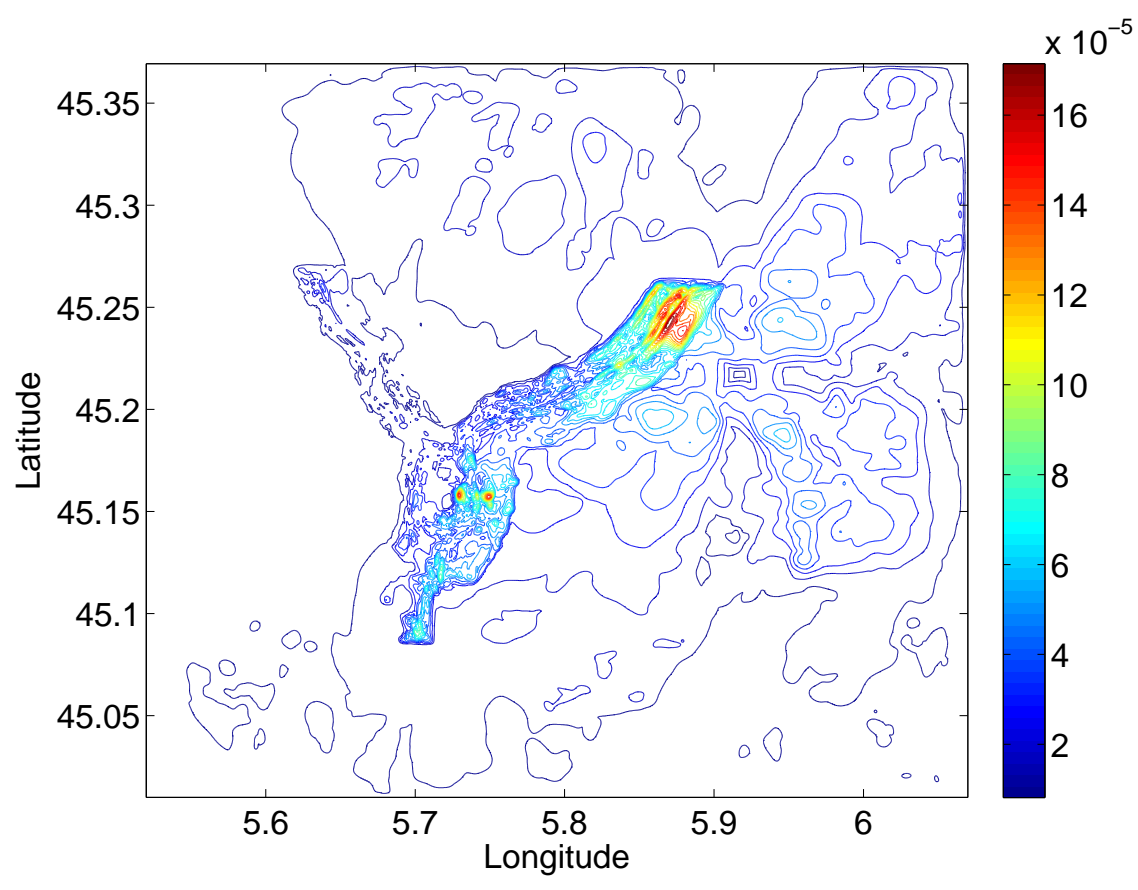

Figure 9.5: Grenoble scenario $M_{w}=2.9$ earthquake. Peak horizontal velocity $[\mathrm{m} / \mathrm{s}]$ for lowpass filtered motion with corner frequecy $f_{c}=1.0 \mathrm{~Hz}$. Note the elevated velocity levels in the sedimentary basin.
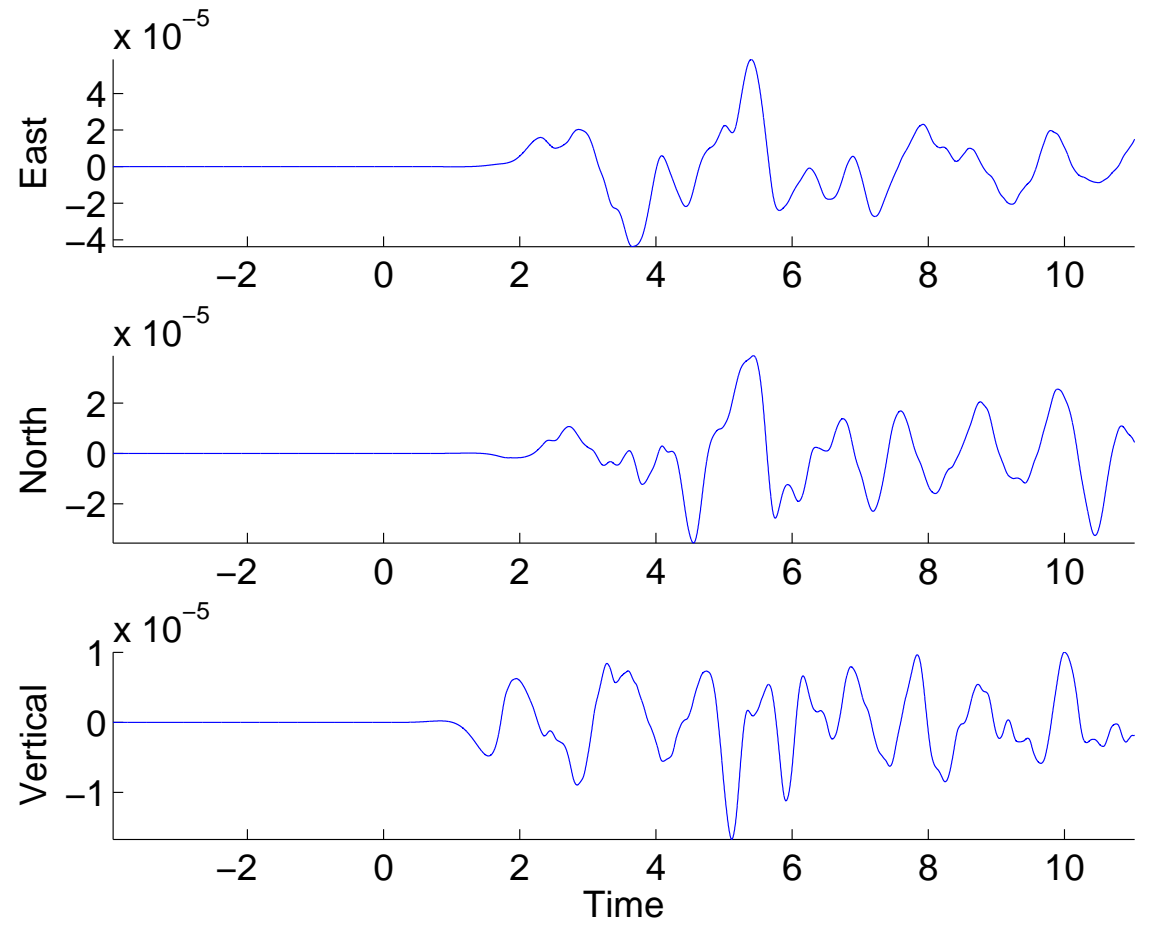

Figure 9.6: Grenoble scenario $M_{w}=2.9$ earthquake. Velocity time history at reciever station R06 located at $(l o n=5.8210$, lat=45.2086, depth=0). 
will take about 16 times longer to execute once the material properties have been read from the Etree. Note that the Etree can take a long time to read, so be patient while the material model is being setup.

The source model for this magnitude $M_{w} \approx 5.4$ earthquake is discretized by many moment tensor sources distributed over the fault plane with variable strength and initiation times. Similar to previous examples, the input files are setup to save several image and sac files. As an example, in Figures 9.7-9.8, we show peak horizontal velocities along the top surface as well as the time history of the motion. The reader is encouraged to run this case by him/herself to further explore the results. If you have access to a larger parallel machine, you can easily capture higher frequencies in the motion by reducing the grid size in the grid command and increasing the corner frequency in the prefilter command.

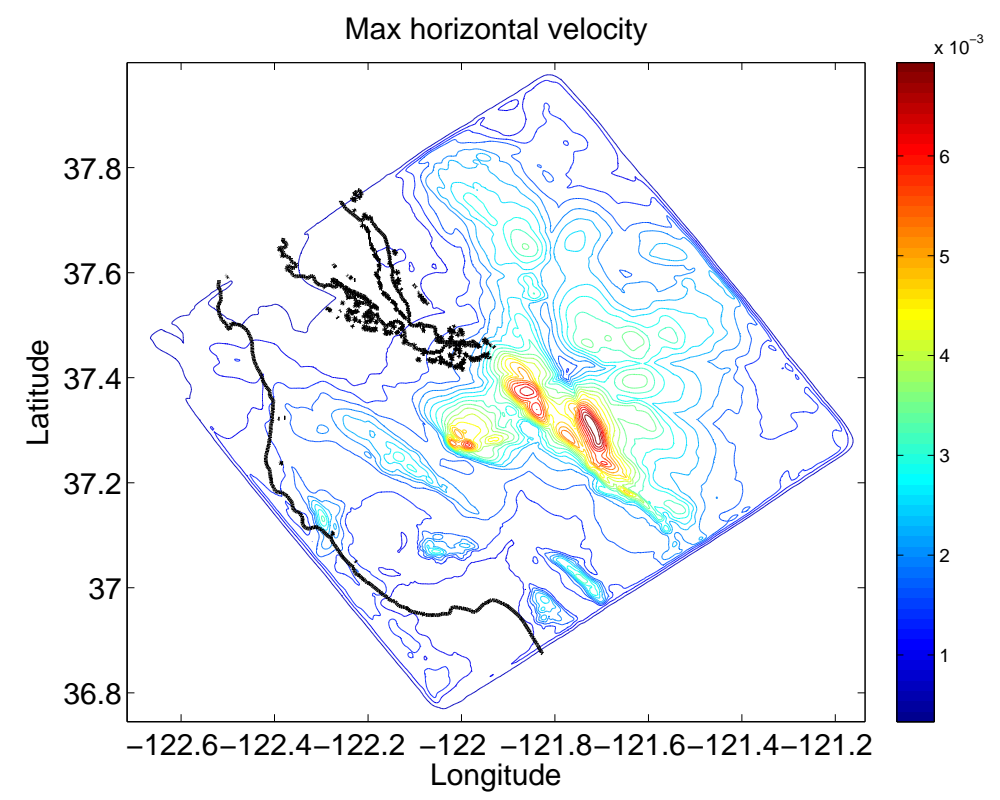

Figure 9.7: Alum Rock $M_{w} \approx 5.4$ earthquake. Max horizontal velocity for lowpass filtered motion with corner frequecy $f_{c}=0.2 \mathrm{~Hz}$. The coast line of southern San Francisco bay and the Pacific ocean is outlined with a thicker black line. 

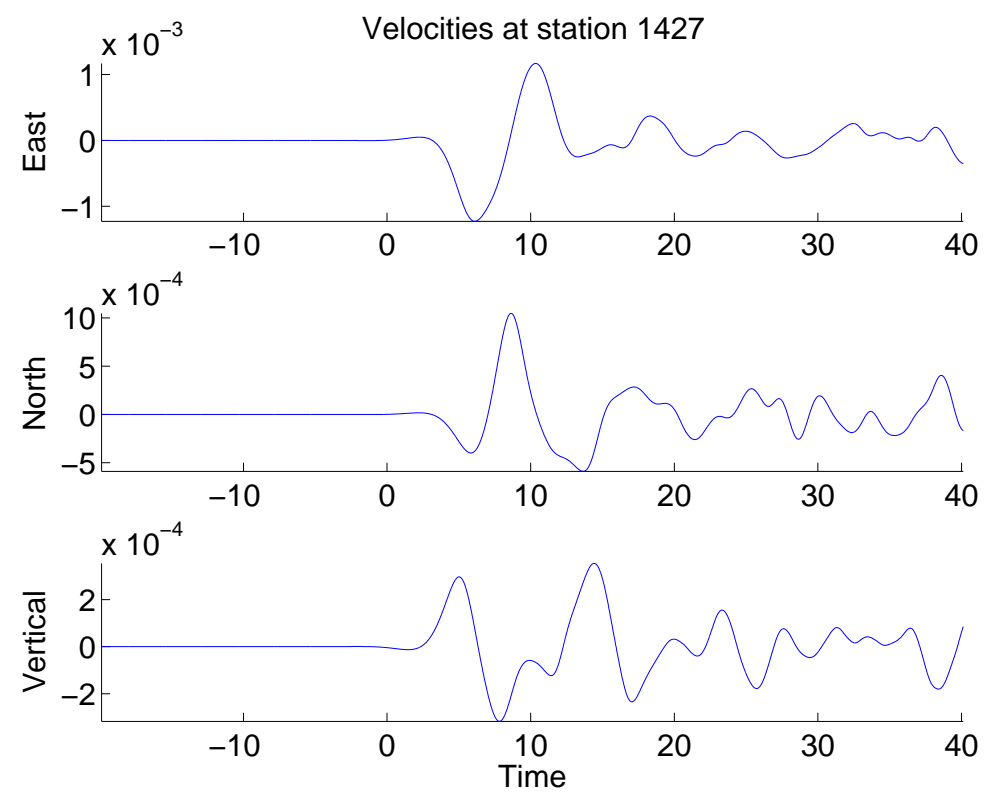

Figure 9.8: Alum Rock $M_{w} \approx 5.4$ earthquake. Simulated velocity time history at station 1427 (lon=122.025, lat=37.402, depth=0). 


\section{Chapter 10}

\section{Keywords in the input file}

The syntax of the input file is

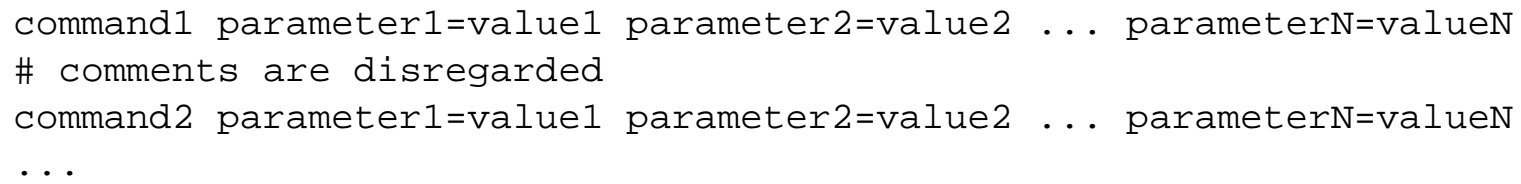

Each command starts at the beginning of the line and ends at the end of the same line. Blank and comment lines are disregarded. A comment is a line starting with a \# character. The order of the parameters within each command is arbitrary. The material commands (block, ifile, pfile, and efile) are applied in the order they appear, but the ordering of all other commands is inconsequential. Also note that the entire input file is read before the simulation starts.

Parameter values are either integers $(-2,0,5, \ldots)$, floating point numbers $(20.5,-0.05,3.4 \mathrm{e} 4)$, or strings (wpp, earthquake, my-favorite-simulation). Note that there must be no spaces around the $=$ signs and strings are given without quotation marks and must not contain spaces. Depending on the specific command, some parameter values are required to fall within specified ranges.

A breif description of all commands is given in the following sections. The commands marked as [required] must be present in all WPP input files, while those marked as [optional] are just that. Other commands, such as those specifying the material model can be given by (a combination of) different commands (block, pfile, efile, or ifile). Unless WPP is run in one of its test modes, the material must be specifed by at least one of these commands and at least one source must be specified.

\subsection{Basic commands}

\subsection{1 fileio [optional]}

\section{Syntax:}

fileio path=... verbose=.. printcycle=.. pfs=.. nwriters=.. Required parameters:

None 


\begin{tabular}{|l|l|l|l|}
\hline \multicolumn{3}{|c|}{ fileio command parameters } \\
\hline Option & Description & Type & Default \\
\hline \hline path & path to a directory where all output will be written & string & . \\
\hline verbose & $\begin{array}{l}\text { sets the level of diagnostic messages written to stan- } \\
\text { dard out }\end{array}$ & int & 0 \\
\hline printcycle & sets the interval for printing the cycle, time, dt info & int & 100 \\
\hline pfs & $\begin{array}{l}\text { assume a parallel (1) or serial (0) file system when } \\
\text { writing image files (several processes can simulta- } \\
\text { neously write the same file on a parallel file system) }\end{array}$ & int & 0 \\
\hline nwriters & set the number of processes that write an image file & int & 8 \\
\hline
\end{tabular}

\subsection{2 grid [required]}

\section{Syntax:}

grid $n x=\ldots \quad n y=\ldots \quad n z=\ldots \quad x=\ldots \quad y=\ldots \quad z=\ldots \quad h=\ldots \quad l a t=\ldots$ lon $=\ldots$ a $\mathrm{z}=\ldots$

Required parameters:

See below.

The grid command specifies the extent of the computational domain and the grid size in the base grid. When grid refinement is used, the base grid is the coarsest grid. Optionally the grid command also specifies the latitude and longitude of the origin and the azimuth angle between North and the $x$-axis.

There are three basic ways of specifying the extent of the computational domain and the grid size:

- number of grid points in all three dimensions and the grid size: $\mathbf{n x}=\ldots \mathbf{n y}=\ldots \mathbf{n z}=\ldots \mathbf{h}=\ldots$

- spatial extents in all three dimensions and the grid size: $\mathbf{x}=\ldots \mathbf{y}=\ldots \mathbf{z}=\ldots \mathbf{h}=\ldots$

- spatial extents in all three dimensions and the number of grid points in one direction (the $\mathrm{x}$-direction in this example): $\mathbf{x}=\ldots \mathbf{y}=\ldots \mathbf{z}=\ldots \mathbf{n x}=\ldots$

It is not allowed to over specify the grid size. For example, if $\mathbf{x}=\ldots$ is given, you can not specify both $\mathbf{h}=. .$. and $\mathbf{n x}=. .$. Similarly, it is not allowed to over specify the extent of the computational domain. For example, when $\mathbf{h}=\ldots$ is given, you can not prescribe both $\mathbf{y}=\ldots$ and $\mathbf{n y}=\ldots$... 


\begin{tabular}{|c|c|c|c|c|}
\hline \multicolumn{5}{|c|}{ grid command parameters } \\
\hline Option & Description & Type & Units & Default \\
\hline $\mathrm{x}$ & physical dimension of grid in the $\mathrm{x}$-direction & float & $\mathrm{m}$ & none \\
\hline $\mathrm{y}$ & physical dimension of grid in the y-direction & float & $\mathrm{m}$ & none \\
\hline $\mathrm{z}$ & physical dimension of grid in the z-direction & float & $\mathrm{m}$ & none \\
\hline h & grid spacing & float & $\mathrm{m}$ & none \\
\hline $\mathrm{nx}$ & number of grid points in the $\mathrm{x}$-direction & int & none & none \\
\hline ny & number of grid points in the y-direction & int & none & none \\
\hline $\mathrm{nz}$ & number of grid points in the z-direction & int & none & none \\
\hline$a z$ & clockwise angle from North to the $\mathrm{x}$-axis & float & degrees & 135.0 \\
\hline lat & latitude geographic coordinate of the origin & float & degrees & 37.0 \\
\hline lon & longitude geographic coordinate of the origin & float & degrees & -118.0 \\
\hline
\end{tabular}

\subsection{3 time [required]}

\section{Syntax:}

time $t=\ldots$ steps $=\ldots$

\section{Required parameters:}

$t$ or steps

The time command specifies the duration of the simulation in seconds or the number of time-steps. The size of the time step is computed internally by WPP. You can not over specify the duration of the simulation, i.e., you can not give both $\mathbf{t}=\ldots$ and $\mathbf{s t e p s}=. .$.

\begin{tabular}{|l|l|l|l|l|}
\hline \multicolumn{5}{|c|}{ time command parameters } \\
\hline Option & Description & Type & Units & Default \\
\hline \hline $\mathrm{t}$ & duration of simulation & float & s & none \\
\hline steps & number of cycles (time-steps) to advance & int & none & none \\
\hline
\end{tabular}

\subsection{4 source [required]}

\section{Syntax:}

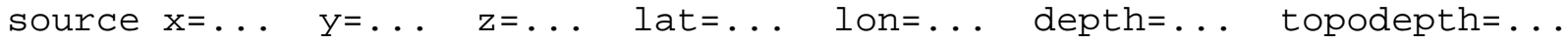

$\mathrm{m} 0=\ldots \quad \mathrm{mxx}=\ldots \quad \mathrm{mxy}=\ldots \quad \mathrm{mxz}=\ldots \quad \mathrm{myy}=\ldots \quad m \mathrm{mz}=\ldots \quad \mathrm{mzz}=\ldots \quad \mathrm{f} 0=\ldots$

$f_{x}=\ldots \quad f_{y}=\ldots \quad f_{z}=\ldots \quad$ rake $=\ldots \quad$ strike $\ldots \quad$ dip $=\ldots \quad$ to $=\ldots$ freq $=\ldots$ type $=\ldots \quad$ ncyc $=\ldots$

\section{Required parameters:}

See below.

There can be multiple source commands in an input file. Each source command either sets up a point force or a point moment tensor source and should follow the following rules: 
- The location of the source must be specified by either a Cartesian location $(\mathbf{x}, \mathbf{y}, \mathbf{z})$ or by geographical coordinates (lat, lon) together with (depth or topodepth). Here depth equals the $\mathbf{z}$-coordinate, while topodepth specifies the depth below the topography.

- Select a point force or a point moment tensor source:

- Point force: give at least one component of the force vector $(\mathbf{f x}, \mathbf{f y}, \mathbf{f z})$ and optionally the amplitude f0.

- A point moment tensor source can be specified in one of two ways:

1. Seismic moment mo, and double couple focal mechanism, strike/dip/rake angles (see [1]).

2. At least one component of the moment tensor $(\mathbf{m x x}, \mathbf{m x y}$, etc.) and optionally a scaling factor $\mathbf{m 0}$.

\begin{tabular}{|c|c|c|c|c|}
\hline \multicolumn{5}{|c|}{ source command parameters } \\
\hline Option & Description & Type & Units & Default \\
\hline $\mathrm{x}$ & $\mathrm{x}$ position of the source & float & $\mathrm{m}$ & none \\
\hline $\mathrm{y}$ & $y$ position of the source & float & $\mathrm{m}$ & none \\
\hline $\mathrm{Z}$ & $\mathrm{z}$ position of the source & float & $\mathrm{m}$ & none \\
\hline depth & depth of the source (below $\mathrm{z}=0$ ) & double & $\mathrm{m}$ & none \\
\hline topodepth & depth of the source (below free surface) & double & $\mathrm{m}$ & none \\
\hline lat & latitude geographic coordinate of the source & double & degrees & none \\
\hline lon & longitude geographic coordinate of the source & double & degrees & none \\
\hline $\mathrm{m} 0$ & moment amplitude & float & $\mathrm{Nm}$ & 1.0 \\
\hline $\operatorname{mxx}$ & $\mathrm{xx}$-component of the moment tensor & float & none & 0.0 \\
\hline myy & yy-component of the moment tensor & float & none & 0.0 \\
\hline $\mathrm{mzz}$ & zZ-component of the moment tensor & float & none & 0.0 \\
\hline mxy & xy-component of the moment tensor & float & none & 0.0 \\
\hline $\mathrm{mxz}$ & $\mathrm{xz}$-component of the moment tensor & float & none & 0.0 \\
\hline myz & yz-component of the moment tensor & float & none & 0.0 \\
\hline f0 & point force amplitude & float & $\mathrm{N}$ & 1.0 \\
\hline fx & forcing function in the $\mathrm{x}$ direction & float & none & 0.0 \\
\hline fy & forcing function in the y direction & float & none & 0.0 \\
\hline $\mathrm{fz}$ & forcing function in the $\mathrm{z}$ direction & float & none & 0.0 \\
\hline strike & Aki and Richards strike angle & float & degrees & none \\
\hline $\operatorname{dip}$ & Aki and Richards dip angle & float & degrees & none \\
\hline rake & Aki and Richards rake angle & float & degrees & none \\
\hline $\mathrm{t} 0$ & offset in time & float & s & 0.0 \\
\hline freq & frequency & float & $\mathrm{Hz}$ or $\mathrm{rad} / \mathrm{s}$ & 1.0 \\
\hline type & selects a particular time dependent function & string & none & RickerInt \\
\hline
\end{tabular}


Options for the time function (type) are: GaussianInt, Gaussian, RickerInt, Ricker, Ramp, Triangle, Sawtooth, Smoothwave, VerysmoothBump, Brune, BruneSmoothed, and Liu.

\subsection{5 prefilter [optional]}

Syntax:

prefilter $f_{C}=\ldots$ maxfreq $=\ldots$

Required parameters:

None

The prefilter command modifies the time functions in all source commands. If the maxfreq parameter is given, the freq parameter in all time function is first limited by this value. If the fc parameter is given, all source time functions are then filter by a 2-pole 2-pass acausal Butterworth filter. In order to avoid an abrupt start, a minimum threshold on the to parameter in all source time functions is set to be $6 / \mathbf{f c}$ before the filtering is performed. The prefilter command can be used to ensure that the solution is well resolved on the computational grid, and is particularly useful for computing reliable hvelmax and vvelmax image files.

\begin{tabular}{|l|l|l|l|l|}
\hline \multicolumn{5}{|c|}{ prefilter command parameters } \\
\hline Option & Description & Type & Units & Default \\
\hline \hline fc & $\begin{array}{l}\text { corner frequency in Butterworth filtering of all } \\
\text { source time functions }\end{array}$ & float & $\mathrm{Hz}$ & None \\
\hline maxfreq & $\begin{array}{l}\text { Enforce a max threshold value in the freq parameter } \\
\text { in all sources }\end{array}$ & float & $\mathrm{Hz}$ or rad/s & None \\
\hline
\end{tabular}

\subsection{The material model [required]}

It is required to define the material model in the entire comutational domain. This can be accomplished by using one or more of the commands in this section.

\subsection{1 block}

\section{Syntax:}

block $v p=\ldots \quad v s=\ldots \quad r h o=\ldots \quad \operatorname{vpgrad}=\ldots \quad \operatorname{vsgrad}=\ldots \quad$ rhograd=... $\mathrm{x} 1=\ldots \quad \mathrm{x} 2=\ldots \quad \mathrm{y} 1=\ldots \quad \mathrm{y} 2=\ldots \quad \mathrm{z} 1=\ldots \quad \mathrm{z} 2=\ldots$

Required parameters:

vp, vs, rho

The block command specifies material properties that are constant or vary linearly with depth. By default, the material properties apply to the entire computational domain. By using the optional parameters $\mathbf{x} 1=. .$. , $\mathbf{x} 2=\ldots$.., etc., the material properties are only assigned in parts of the computational domain. When used together with the topography command, $\mathbf{z 1}=\ldots$ and $\mathbf{z 2}=\ldots$ specify depths below the free surface rather than $z$-coordinate.

The gradient parameters vpgrad, vsgrad, and rhograd specify linear variations in the $z$-direction (downward). The units for vpgrad and vsgrad are 1/seconds, which can be interpreted as $\mathrm{m} / \mathrm{s}$ per $\mathrm{m}$, or 
$\mathrm{km} / \mathrm{s}$ per $\mathrm{km}$. The linear variation is relative to the properties at the free surface $(z=0$ or depth=0 with topography), e.g.,

$$
V_{p}(z)=\mathbf{v p}+z \mathbf{v p g r a d} .
$$

Note that when vpgrad is specified together with $\mathbf{z} \mathbf{1}=z_{1}, V_{p}\left(z_{1}\right)=\mathbf{v p}+z_{1} \mathbf{v p g r a d}$. Hence, the material properties at the top of the block $\left(z=z_{1}\right)$ can be very different from $\mathbf{v p}$ when $z_{1}$ vpgrad is large.

\begin{tabular}{|l|l|l|l|l|}
\hline \multicolumn{5}{|c}{ block command parameters } \\
\hline Option & Description & Type & Units & Default \\
\hline \hline vp & P-wave velocity & float & $\mathrm{m} / \mathrm{s}$ & none \\
\hline vs & S-wave velocity & float & $\mathrm{m} / \mathrm{s}$ & none \\
\hline rho & density & float & $\mathrm{kg} / \mathrm{m}^{3}$ & none \\
\hline vpgrad & vertical gradient for vp & float & $\mathrm{s}^{-1}$ & none \\
\hline vsgrad & vertical gradient for vs & float & $\mathrm{s}^{-1}$ & none \\
\hline rhograd & vertical gradient for rho & float & $\mathrm{kg} / \mathrm{m}^{4}$ & none \\
\hline x1 & minimum x-dim for the box shaped sub-region & float & $\mathrm{m}$ & -max x \\
\hline x2 & maximum x-dim for the box shaped sub-region & float & $\mathrm{m}$ & 2 max x \\
\hline \hline y1 & minimum y-dim for the box shaped sub-region & float & $\mathrm{m}$ & -max y \\
\hline y2 & maximum y-dim for the box shaped sub-region & float & $\mathrm{m}$ & 2 max y \\
\hline \hline z1 & minimum z-dim for the box shaped sub-region & float & $\mathrm{m}$ & -max z \\
\hline z2 & maximum z-dim for the box shaped sub-region & float & $\mathrm{m}$ & 2 max z \\
\hline
\end{tabular}

\subsection{2 efile}

\section{Syntax:}

efile etree=... xetree=... $\log$ xile=... query=... vsmin=.. vpmin $=\ldots \quad$ access $=\ldots$ resolution $=\ldots$

\section{Required parameters:}

etree

\begin{tabular}{|l|l|l|l|l|}
\hline \multicolumn{5}{|c|}{ efile command parameters } \\
\hline Option & Description & Type & Units & Default \\
\hline \hline logfile & $\begin{array}{l}\text { name of file where output from etree file read will } \\
\text { go }\end{array}$ & string & none & none \\
\hline vsmin & minimum threshold for the s velocity in solids & float & $\mathrm{m} / \mathrm{s}$ & 0 \\
\hline vpmin & minimum threshold for the p velocity in solids & float & $\mathrm{m} / \mathrm{s}$ & 0 \\
\hline \hline query & type of query to perform & string & none & MAXRES \\
\hline resolution & for FIXEDRES, the resolution to sample the data & float & $\mathrm{m}$ & none \\
\hline access & can be set to parallel or serial & string & & parallel \\
\hline \hline etree & full path to the etree database file & string & none & none \\
\hline xetree & full path to the extended etree database file & string & none & none \\
\hline
\end{tabular}


The query option can be set to one of the following:

\begin{tabular}{ll}
\hline Query Option & Description \\
\hline MAXRES & $\begin{array}{l}\text { This will sample the data at the maximum resolution available in the database, } \\
\text { which is the default query type for the efile option. }\end{array}$ \\
FIXEDRES & $\begin{array}{l}\text { This will sample the database at the requested resolution, even if the database } \\
\text { contains values at a higher resolution. This option defaults to the grid spacing } \\
\text { h, or can be specified with the resolution option. }\end{array}$
\end{tabular}

For example, to set the data to be fixed at a $1 \mathrm{~km}$ sampling:

efile query=FIXEDRES resolution=1000 etree=USGS-SF1906. etree

Note: If you would like to find out the locations of grid points which are found to be outside the etree database domain, the logfile option can be used to track which points were not found. It will report points it found outside the domain, as well as points it did not have data for (i.e., air just above water or a material).

\subsection{3 pfile}

\section{Syntax:}

pfile smoothingsize=.. vpmin=.. vsmin=.. $\quad$ rhomin=.. flatten=... filename=... directory $=\ldots$.

\section{Required parameters:}

filename

\begin{tabular}{|l|l|l|l||l|}
\hline \multicolumn{5}{|c|}{ pfile command parameters } \\
\hline Option & Description & Type & Units & Default \\
\hline \hline filename & name of input pfile & string & none & none \\
\hline directory & name of directory for the input pfile & string & none & . \\
\hline smoothingsize & smooth data over stencil of this width & int & none & 5 \\
\hline vpmin & minimum threshold value for $V_{P}$ & float & $\mathrm{m} / \mathrm{s}$ & 0 \\
\hline vsmin & minimum threshold value for $V_{S}$ & float & $\mathrm{m} / \mathrm{s}$ & 0 \\
\hline rhomin & minimum threshold value for density & float & $\mathrm{m} / \mathrm{s}$ & 0 \\
\hline flatten & Flatten earth model (T or F) & string & none & $\mathrm{F}$ \\
\hline
\end{tabular}

\subsection{4 ifile}

\section{Syntax:}

ifile filename=...

\section{Required parameters:}

\section{filename}

The ifile command specifies the depth of material surfaces as function of longitude and latitude, and must be used in conjunction with the material command. The format for this file is described in Section 11.3. 


\begin{tabular}{|l|l|c|l|}
\hline \multicolumn{3}{|c|}{ ifile command parameters } \\
\hline Option & Description & Type & Default \\
\hline \hline filename & name of input file holding material surfaces & string & None \\
\hline
\end{tabular}

\subsection{5 material}

\section{Syntax:}

material $i d=\ldots \quad v p=\ldots \quad v s=\ldots \quad r h o=\ldots \quad$ vpgrad=... vsgrad=... rhograd $=\ldots \quad$ vp $2=\ldots \quad$ vs $2=\ldots \quad$ rho $2=\ldots$

Required parameters:

id, vp, vs, rho

The material command is used to define material properties together with the ifile material surfaces, see Section 11.3.

\begin{tabular}{|l|l|l|l|}
\hline \multicolumn{3}{|c|}{ material command parameters } \\
\hline Option & Description & Type & Default \\
\hline \hline id & material ID number $>0$ & int & None \\
\hline vp & P-wave velocity & float & None \\
\hline vs & S-wave velocity & float & None \\
\hline rho & Density & float & None \\
\hline vpgrad & P-velocity gradient & float & 0.0 \\
\hline vsgrad & S-velocity gradient & float & 0.0 \\
\hline rhograd & Density gradient & float & 0.0 \\
\hline vp2 & P-velocity quadratic coefficient & float & 0.0 \\
\hline vs2 & S-velocity quadratic coefficient & float & 0.0 \\
\hline rho2 & Density quadratic coefficient & float & 0.0 \\
\hline
\end{tabular}

\subsection{6 globalmaterial [optional]}

\section{Syntax:}

globalmaterial vpmin=... vsmin=...

Required parameters:

None

The globalmaterial command is used to put threshold values on the $P$ - and $S$-velocities in the material model. These thresholds are enforced after material properties have been assigned to all grid points.

\begin{tabular}{|l|l|l|l|}
\hline \multicolumn{3}{|c|}{ globalmaterial command parameters } \\
\hline Option & Description & Type & Default \\
\hline \hline vpmin & Minimum P-wave velocity $(>0)$ & float & None \\
\hline vsmin & Minimum S-wave velocity $(>0)$ & float & None \\
\hline
\end{tabular}




\subsection{Topography and mesh refinement}

\subsection{1 topography [optional]}

\section{Syntax:}

topography input=... file=... resolution=... zmax=... order=... smooth=... gaussianAmp $=\ldots$ gaussianXc=... gaussianYc=... gaussianLx=... gaussianLy=...

\section{Required parameters:}

input, zmax

Alse see discussion below.

The topography command specifies the shape of the free surface boundary, the vertical extent of the curvilinear grid below the free surface, and optionally the order of the grid mapping. The topography is given as elevation (in meters) relative to mean sea level, i.e., positive above sea level and negative below sea level. The curvilinear grid is located between the topography and $z=z_{\max }$ (recall that $z$ is directed downwards). If the elevation of the topography ranges between $z=-e_{\min }$ and $z=-e_{\max }$, we recommend using $z_{\max } \geq-e_{\min }+2\left|e_{\max }-e_{\min }\right|$.

There are three ways to specify the topography:

- input=file Read the topography as function of latitude and longitude. The file name must be specified by the file $=. .$. parameter. The format for this file is described in Section 11.1.

- input=efile Read the topography from the Etree data base. The Etree data base must be specified by an efile command (see below). The spatial resolution for querying the Etree data base can be specified by the resolution $=. .$. parameter.

- input=gaussian Build an analytical topography in the shape of a Gaussian hill. The amplitude is specified by gaussianAmp=..., the hill is centered at gaussianXc=..., gaussianYc=..., and the half width of the hill in the $x$ and $y$-directions are specified by gaussian $\mathbf{L x}=\ldots$.., and gaussianLy=....

\begin{tabular}{|l|l|l|l|l|}
\hline \multicolumn{5}{|c|}{ topography command parameters } \\
\hline Option & Description & Type & Units & Default \\
\hline \hline input & Type of input: file, efile or gaussian & string & none & none \\
\hline file & File name if input=file & string & none & none \\
\hline resolution & Resolution for querying the efile if input-efile & float & meters & none \\
\hline zmax & $\begin{array}{l}\text { z coordinate of the interface between Cartesian and } \\
\text { curvilinear grid }\end{array}$ & float & m & 0 \\
\hline order & Interpolation order $(2,3$ or 4$)$ & int & none & 3 \\
\hline smooth & $\begin{array}{l}\text { Number of smoothing iterations of topography grid } \\
\text { surface }\end{array}$ & int & none & 10 \\
\hline gaussianAmp & Amplitude for a Gaussian hill topography & float & meters & 0.05 \\
\hline gaussianXc & x-coordinate of center for a Gaussian Hill & float & meters & 0.5 \\
\hline gaussianYc & y-coordinate of center for a Gaussian Hill & float & meters & 0.5 \\
\hline gaussianLx & Width of the Gaussian hill in the x-direction & float & meters & 0.15 \\
\hline gaussianLy & Width of the Gaussian hill in the y-direction & float & meters & 0.15 \\
\hline
\end{tabular}




\subsection{2 refinement [optional]}

Each refinement command corresponds to a mesh refinement patch for $z \leq$ zmax. The grid size in each refinement patch is half of the next coarser grid size. The grid size in the coarsest grid is prescribed by the grid command.

\section{Syntax:}

refinement $z \max =\ldots$

Required parameters:

zmax

\begin{tabular}{|l|l|l|l|l|}
\hline \multicolumn{5}{|c|}{ refinement command parameters } \\
\hline Option & Description & Type & Unit & Default \\
\hline \hline zmax & maximum z-coordinate for the refinement region & float & $\mathrm{m}$ & None \\
\hline
\end{tabular}

\subsection{Output commands}

The output commands are optional but unless you are only doing timing studies of the WPP code, you probably want some data to be saved. The sac command saves a time series of the solution at a recording station, which can be read by the SAC program [4] or the readsac.m Matlab script in the tools directory. The image command is used to save a two-dimensional cross-section of the solution, the material properties, or the grid. The image files can be read by the readimagepatch.m Matlab script in the tools directory. The gmt command outputs a shell script file containing the location of all sac stations and the epicenter, i.e. the location of the first source command. This shell script file can be used for further postprocessing by the GMT program [11].

\subsection{1 sac [optional]}

\section{Syntax:}

$\operatorname{sac}=\ldots \quad x=\ldots \quad y=\ldots \quad z=\ldots \quad l a t=\ldots \quad l o n=\ldots \quad \operatorname{depth=\ldots }$

topodepth=... sta=... file=... type=... writeEvery=...

eventDate=... eventTime=... nsew=... velocity=.. usgsformat=...

sacformat $=\ldots$

Required parameters:

Location of the receiver in Cartesian or geographic coordinates.

The file format is described in Section 11.4. 


\begin{tabular}{|l|l|l|l|l|}
\hline \multicolumn{5}{|c}{ sac command parameters } \\
\hline Option & Description & Type & Units & Default \\
\hline \hline x & x position of the receiver & float & m & none \\
\hline y & y position of the receiver & float & m & none \\
\hline z & z position of the receiver & float & m & none \\
\hline \hline lat & latitude geographic coordinate of the receiver & float & degrees & none \\
\hline lon & longitude geographic coordinate of the receiver & float & degrees & none \\
\hline depth & depth of the receiver (below topography) & float & m & none \\
\hline topodepth & depth of the receiver (same as depth) & float & m & none \\
\hline \hline sta & name of the station & string & none & file \\
\hline file & file name header of the SAC file & string & none & sac \\
\hline type & write out a binary or ascii SAC file & string & none & binary \\
\hline writeEvery & cycle interval to write out the SAC file to disk & int & none & 1000 \\
\hline \hline eventDate & date the event occured: YYYY/MM/DD & int/int/int & none & date of run \\
\hline eventTime & time the event occured: hours:minutes:seconds & int:int:int & none & time of run \\
\hline \hline nsew & $\begin{array}{l}\text { output East-West, North-South, and vertical }(-z) \\
\text { components }\end{array}$ & int & none & 0 \\
\hline velocity & output time derivative of solution & int & none & 0 \\
\hline usgsformat & output all components in an ASCII text file & int & none & 0 \\
\hline sacformat & output each component in a SAC file & int & none & 1 \\
\hline
\end{tabular}

\subsection{2 image [optional]}

\section{Syntax:}

image $=\ldots \quad x=\ldots \quad y=\ldots \quad z=\ldots \quad$ time=.. timeInterval=.. cycle=.. cycleInterval=... file=... mode=... precision=...

Required parameters:

Location of the image slice $(x, y$, or $z)$

Timing interval (time, timeInterval, cycle, or cycleInterval)

The file format is described in Section 11.5. 


\begin{tabular}{|l|l|l|l|l|}
\hline \multicolumn{5}{|c|}{ image command parameters } \\
\hline Option & Description & Type & Units & Default \\
\hline \hline $\mathrm{x}$ & x location of visual plane & float & $\mathrm{m}$ & none \\
\hline $\mathrm{y}$ & y location of visual plane & float & $\mathrm{m}$ & none \\
\hline $\mathrm{z}$ & z location of visual plane & float & $\mathrm{m}$ & none \\
\hline \hline time & $\begin{array}{l}\text { simulation time to output image, will be closest de- } \\
\text { pending on dt taken }\end{array}$ & float & $\mathrm{s}$ & none \\
\hline timeInterval & simulation time interval to output series of images & float & s & none \\
\hline cycle & time-step cycle to output image & int & none & none \\
\hline cycleInterval & time-step cycle interval to output a series of images & int & none & none \\
\hline file & file name header of image & string & none & image \\
\hline mode & specifies which field is written to the image file & string & none & rho \\
\hline precision & precision of image data on file (float or double) & string & none & float \\
\hline
\end{tabular}

mode can take one of the following values: 


\begin{tabular}{|c|l|}
\hline \multicolumn{2}{|l|}{ image mode options } \\
\hline Value & Description \\
\hline \hline ux & displacement in the x-direction \\
\hline uy & displacement in the y-direction \\
\hline uz & displacement in the z-direction \\
\hline rho & density \\
\hline lambda & lambda \\
\hline mu & mu \\
\hline $\mathrm{p}$ & p velocity \\
\hline $\mathrm{s}$ & s velocity \\
\hline div & divergence (div) of the displacement \\
\hline curl & magnitude of the rotation (curl) of the displacement \\
\hline veldiv & divergence (div) of the velocity \\
\hline velcurl & magnitude of the rotation (curl) of the velocity \\
\hline velmag & magnitude of the velocity \\
\hline lat & latitude (in degrees) \\
\hline lon & longitude (in degrees) \\
\hline hvelmax & maximum in time of the horizontal velocity (North-East components) \\
\hline vvelmax & maximum in time of the vertical velocity \\
\hline topo & topography or elevation [only available with efile input $]$ \\
\hline grid & grid coordinates in the plane of visualization (e.g. $\mathrm{y}$-z plane if $\mathrm{x}=\mathrm{const}$ ) \\
\hline uxerr & error between computed and exact solutions in the x-direction \\
\hline uyerr & error between computed and exact solutions in the $\mathrm{y}$-direction \\
\hline uzerr & error between computed and exact solutions in the z-direction \\
\hline fx & Forcing in the x-direction \\
\hline fy & Forcing in the y-direction \\
\hline fz & Forcing in the z-direction \\
\hline
\end{tabular}

\subsection{3 gmt [optional]}

\section{Syntax:}

gmt file=...

\section{Required parameters:}

None.

\begin{tabular}{|l|l|l|l|}
\hline \multicolumn{3}{|c|}{ gmt command parameters } \\
\hline Option & Description & Type & Default \\
\hline \hline file & name of output file for gmt c-shell commands & string & wpp.gmt.csh \\
\hline
\end{tabular}




\subsection{WPP testing commands [optional]}

\subsection{1 twilight}

\section{Syntax:}

twilight=... errorlog=... omega=... $\quad c=\ldots \quad$ phase=.. momega=... mphase $=\ldots$ amprho $=\ldots \quad$ ampmu $=\ldots \quad$ amplambda $=\ldots$

\section{Required parameters:}

None

The twilight command runs $W P P$ in a testing mode where forcing functions are constructed to create a known smooth analytical solution. The error in this solution should be $\mathcal{O}\left(h^{2}\right)$ when this solution is sufficiently well resolved on the computational grid. Example scripts are provided in examples/twilight.

\begin{tabular}{|l|l|l|l|}
\hline \multicolumn{4}{|c|}{ twilight command parameters } \\
\hline Option & Description & Type & Default \\
\hline \hline errorlog & Outputs error log in file twilight_errors.dat & int & 0 \\
\hline omega & Wave number in solution & float & 1.0 \\
\hline c & Wave speed & float & 1.3 \\
\hline phase & Solution phase coefficient & float & 0.0 \\
\hline momega & Wave number in material & float & 1.0 \\
\hline mphase & Material phase coefficient & float & 0.4 \\
\hline amprho & Density amplitude & float & 1.0 \\
\hline ampmu & Material $\mu$ amplitude & float & 1.0 \\
\hline amplambda & Material $\lambda$ amplitude & float & 1.0 \\
\hline
\end{tabular}

\subsection{2 testlamb}

\section{Syntax:}

test lamb=... $\mathrm{x}=\ldots \quad \mathrm{y}=\ldots \quad \mathrm{cp}=\ldots \quad \mathrm{rho}=\ldots \quad \mathrm{fz}=\ldots$

Required parameters:

Location of the forcing $(x, y)$.

The testlamb command solves Lamb's problem, i.e., the displacement due to a vertical point forcing on a flat free surface, located at $(x, y)$. The material has homogeneous properties and the ratio between the compressional and shear velocities is $\sqrt{3}$, i.e., $\lambda=\mu$. Hence the shear velocity is $C_{s}=C_{p} / \sqrt{3}$. Furthermore, the time function is a verysmoothBump with freq $=1$ and $\mathbf{t} \mathbf{0}=0$. Note that the vertical component of the exact solution is available along the free surface $(z=0)$, and images of the error can be saved with the image command using the options type=uzerr $z=0$. At the end of the run, the error in the solution are reported measured in max and $L_{2}$ norms. 


\begin{tabular}{|l|l|l|l|}
\hline \multicolumn{3}{|c|}{ testlamb command parameters } \\
\hline Option & Description & Type & Default \\
\hline \hline $\mathrm{x}$ & x-coordinate of point source & float & 0.0 \\
\hline $\mathrm{y}$ & y-coordinate of point source & float & 0.0 \\
\hline $\mathrm{cp}$ & P-wave velocity & float & 1.0 \\
\hline rho & Density & float & 1.0 \\
\hline fz & Magnitude of the forcing & float & 1.0 \\
\hline
\end{tabular}

\subsection{3 testpointsource}

\section{Syntax:}

testpointsource $\mathrm{x}=\ldots \quad \mathrm{y}=\ldots \quad \mathrm{z}=\ldots \quad \mathrm{cp}=\ldots \quad \mathrm{cs}=\ldots \quad$ rho=.. $\mathrm{m} 0=\ldots$

$\mathrm{mxx}=\ldots \quad \mathrm{mxy}=\ldots \quad \mathrm{mxz}=\ldots \quad \mathrm{myy}=\ldots \quad \mathrm{myz}=\ldots \quad \mathrm{mzz}=\ldots \quad \mathrm{f} 0=\ldots \quad \mathrm{fx}=\ldots$

$f_{y}=\ldots \quad f z=\ldots \quad$ freq $=\ldots \quad t 0=\ldots \quad$ type $=\ldots$

Required parameters:

None

This command solves the displacement due to a point source in a whole space. The reported errors are only reliable before the solution has reached the outflow boundaries. 


\begin{tabular}{|c|c|c|c|}
\hline \multicolumn{4}{|c|}{ testpointsource command parameters } \\
\hline Option & Description & Type & Default \\
\hline $\mathrm{x}$ & $\mathrm{x}$-coordinate of point source & float & 0 \\
\hline $\mathrm{y}$ & $y$-coordinate of point source & float & 0 \\
\hline $\mathrm{Z}$ & z-coordinate of point source & float & 0 \\
\hline $\mathrm{cp}$ & P-wave velocity & float & $\sqrt{3}$ \\
\hline cs & S-wave velocity & float & 1 \\
\hline rho & Density & float & 1 \\
\hline $\mathrm{m} 0$ & Moment amplitude & float & 1 \\
\hline $\operatorname{mxx}$ & $\mathrm{xx}$-component of moment tensor & float & 0 \\
\hline mxy & xy-component of moment tensor & float & 0 \\
\hline $\mathrm{mxz}$ & xz-component of moment tensor & float & 0 \\
\hline myy & yy-component of moment tensor & float & 0 \\
\hline myz & yz-component of moment tensor & float & 0 \\
\hline mzz & zz-component of moment tensor & float & 0 \\
\hline f0 & Point force amplitude & float & 1 \\
\hline fx & Magnitude of the forcing in the $\mathrm{x}$-direction & float & 1 \\
\hline fy & Magnitude of the forcing in the $y$-direction & float & 1 \\
\hline $\mathrm{fz}$ & Magnitude of the forcing in the $\mathrm{z}$-direction & float & 1 \\
\hline freq & Frequency of the forcing & float & 1 \\
\hline $\mathrm{t} 0$ & Offset in time & float & 1 \\
\hline type & $\begin{array}{l}\text { Type of the source: SmoothWave, VerySmooth- } \\
\text { Bump, Ricker }\end{array}$ & string & Ricker \\
\hline
\end{tabular}

\subsection{Advanced simulation controls}

Most users will never need to use the commands in this section.

\subsection{1 supergrid [optional]}

\section{Syntax:}

supergrid thickness=... damping_coefficient=...

\section{Required parameters:}

None 


\begin{tabular}{|l|l|l|l|}
\hline \multicolumn{3}{|c|}{ supergrid command parameters } \\
\hline Option & Description & Type & Default \\
\hline \hline thickness & Thickness of the supergrid region & float & $15 \mathrm{~h}$ \\
\hline damping_coefficient & Damping in supergrid region & float & 0.15 \\
\hline
\end{tabular}

\subsection{2 boundary_conditions [optional]}

\section{Syntax:}

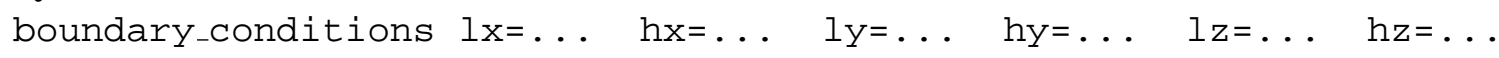

Required parameters:

None

\begin{tabular}{|l|l|l|l|}
\hline \multicolumn{3}{|c}{ Boundary conditions parameters } \\
\hline Option & Description & Type & Default \\
\hline \hline $\mathrm{lx}$ & Boundary condition at $x=0$ & int $0-5$ & 5 \\
\hline $\mathrm{hx}$ & Boundary condition at $x=x_{\max }$ & int $0-5$ & 5 \\
\hline $\mathrm{ly}$ & Boundary condition at $y=0$ & int $0-5$ & 5 \\
\hline $\mathrm{hy}$ & Boundary condition at $y=y_{\max }$ & int $0-5$ & 5 \\
\hline $\mathrm{lz}$ & Boundary condition at $d e p t h=0$ & int $0-5$ & 2 \\
\hline $\mathrm{hz}$ & Boundary condition at $z=z_{\max }$ & int $0-5$ & 5 \\
\hline
\end{tabular}

\begin{tabular}{|l|l|}
\hline \multicolumn{2}{|c|}{ boundary condition type options } \\
\hline Value & Type \\
\hline \hline 0 & Clayton-Enquist boundary \\
\hline 1 & Energy absorbing boundary \\
\hline 2 & Stress-free boundary \\
\hline 3 & Dirichlet boundary \\
\hline 4 & Neumann boundary \\
\hline 5 & Supergrid boundary \\
\hline
\end{tabular}

\subsection{3 developer [optional]}

\section{Syntax:}

developer cfl_number=... output_load=... output_timing=...

interpolation=... ctol=... cmaxit=... log_energy=...

print_energy $=\ldots$ mpiio $=\ldots$ iotiming=...

Required parameters:

None 
Warning: you need to be intimately familiar with the inner workings of WPP to use this command. Look in the source code to get a full understanding of what this command really does.

\begin{tabular}{|l|l|l|l|}
\hline \multicolumn{3}{|c|}{ developer parameters } \\
\hline Option & Description & Type & Default \\
\hline \hline cfl_number & CFL number & float & 0.8 \\
\hline output_load & Output lad info $(0$ or 1$)$ & int & 0 \\
\hline output_timing & Output timing info $(0$ or 1$)$ & int & 0 \\
\hline interpolation & Interpolation type at grid refinement boundaries & string & conservative \\
\hline ctol & $\begin{array}{l}\text { Relative tolerance for iterative solution of conservative grid re- } \\
\text { finement }\end{array}$ & float & $1 \mathrm{e}-3$ \\
\hline cmaxit & $\begin{array}{l}\text { Max number of interations for solving conservative grid refine- } \\
\text { ment equations }\end{array}$ & int & 20 \\
\hline log_energy & File name for saving energy info & string & none \\
\hline print_energy & Save energy information $(0$ or 1$)$ & int & 0 \\
\hline mpiio & $\begin{array}{l}\text { use standard MPI-I/O }(1) \text { or Bjorn's I/O }(0) \text { routines for saving } \\
\text { image files }\end{array}$ & int & 0 \\
\hline iotiming & output timing info after each image is written to disk. (0 or 1$)$ & int & 0 \\
\hline
\end{tabular}




\section{Chapter 11}

\section{File formats}

\section{1 topography}

Topography is specified as elevation above mean sea level on a regular lattice in gegraphic coordinates. The unit for elevation is meters, while latitude and longitude are in degrees. A topography file must cover the entire horizontal extent of the computational domain.

Let the elevation be known at longitudes

$$
\phi_{i}, \quad i=1,2, \ldots, N_{l o n},
$$

and latitudes

$$
\theta_{j}, \quad j=1,2, \ldots, N_{l a t},
$$

Note that the latitudes and the longitudes must either be strictly increasing or strictly decreasing, but the step size may vary.

The elevation should be given on the regular lattice

$$
e_{i, j}=\text { elevation at longitude } \phi_{i} \text {, latitude } \theta_{j} .
$$

The topography file should be an ASCII text file with the following format. The first line of the file holds the number of longitude and latitude data points:

$$
N_{\text {lon }} \quad N_{\text {lat }}
$$

On subsequent lines, longitude, latitude and elevation values are given in column first ordering:

$\begin{array}{ccc}\phi_{1} & \theta_{1} & e_{1,1} \\ \phi_{2} & \theta_{1} & e_{2,1} \\ \vdots & \vdots & \vdots \\ \phi_{\text {Nlon }} & \theta_{1} & e_{\text {Nlon }, 1} \\ \vdots & \vdots & \vdots \\ \phi_{1} & \theta_{\text {Nlat }} & e_{1, \text { Nlat }} \\ \phi_{2} & \theta_{\text {Nlat }} & e_{2, \text { Nlat }} \\ \vdots & \vdots & \vdots \\ \phi_{\text {Nlon }} & \theta_{\text {Nlat }} & e_{\text {Nlon,Nlat }}\end{array}$




\section{2 pfile}

The header has 7 lines and follows the following format:

\begin{tabular}{|c|c|c|c|c|}
\hline Line & Column 1 & Column 2 & Column 3 & Column 4 \\
\hline 1 & Name (string) & & & \\
\hline 2 & $\Delta[\mathrm{deg}]($ real $)$ & & & \\
\hline 3 & $N_{\text {lat }}$ (integer) & Lat $_{\text {min }}[\mathrm{deg}]($ real) & $L a t_{\max }[\mathrm{deg}](\mathrm{real})$ & \\
\hline 4 & $N_{\text {lon }}$ (integer) & $\operatorname{Lon}_{\min }[\mathrm{deg}]$ (real) & $\operatorname{Lon}_{\max }[\mathrm{deg}]$ (real) & \\
\hline 5 & $N_{d e p}$ (integer) & $d_{\min }[\mathrm{km}]($ real $)$ & $d_{\max }[\mathrm{km}](\mathrm{real})$ & \\
\hline 6 & $I_{\text {sed }}$ (integer) & $I_{M o H o}$ (integer) & $I_{410}$ (integer) & $I_{660}$ (integer) \\
\hline 7 & $Q$-available? (logical) & & & \\
\hline
\end{tabular}

Lines 3 and 4 contain the number of lattice points as well as the starting and ending angles in the latitude and longitude direction, respectively, . Line 5 contains the number of depth values in each profile, followed by the minimum and maximum depth measured in $\mathrm{km}$. Line 6 supplies information about the index of some material discontinuities in each depth profile. Give -99 if not known. Note that the index for each discontinuity (sediment, MoHo, 410, 660) indicates the row number within each profile, for the material property just above the discontinuity. Hence, the subsequent entry in each profile should have the same depth value and contain the material property just below the same discontinuity. Line 7 should contain the single letter $\mathrm{T}$ if the subsequent data contains quality factors $\left(Q_{P}\right.$ and $\left.Q_{S}\right)$; otherwise it should contain the single letter $\mathrm{F}$.

The header is directly followed by $N_{l a t} \times N_{\text {lon }}$ depth profiles, according to the pseudo-code

$$
\begin{aligned}
& \text { for } i=0,1, \ldots, N_{l a t}-1 \\
& \text { for } j=0,1, \ldots, N_{l o n}-1 \\
& L a t_{i}=L a t_{\text {min }}+i \Delta ; \\
& \operatorname{Lon}_{j}=\operatorname{Lon}_{\min }+j \Delta \text {; } \\
& \text { (save depth profile for } \mathrm{Lat}_{i}, \mathrm{Lon}_{j} \text { ) } \\
& \text { end }
\end{aligned}
$$

The first line of each depth profile contains its latitude and longitude (in degrees as real numbers), and the number of depth values which must equal $N_{d e p}$. The subsequent $N_{d e p}$ lines have the following format:

\begin{tabular}{llllllll} 
Index (integer) & depth $[\mathrm{km}](\mathrm{real})$ & $V_{p}[\mathrm{~km} / \mathrm{s}](\mathrm{real})$ & $V_{s}[\mathrm{~km} / \mathrm{s}](\mathrm{real})$ & $\rho\left[\mathrm{g} / \mathrm{cm}^{3}\right](\mathrm{real})$ & $Q_{P}(\mathrm{real})$ & $Q_{S}(\mathrm{real})$ \\
\hline
\end{tabular}

Note that $Q_{P}$ and $Q_{S}$ should only be present when indicated so by the $Q$-availability flag on line 7 of the header.

\section{3 ifile}

The material surface file (ifile) should be an ASCII text file with the following format. The first line of the file holds the number of longitude and latitude data points, as well as the number of material surfaces:

$$
N_{\text {lon }} \quad N_{\text {lat }} \quad N_{\text {mat }}
$$


On subsequent lines, longitude, latitude and $N_{\text {mat }}$ surface depth values are given in column first ordering:

\begin{tabular}{|c|c|c|c|c|}
\hline $\operatorname{Lon}_{1}$ & $L^{2} t_{1}$ & $d_{1,1,1}$ & $\ldots$ & $d_{N_{m a t}, 1,1}$ \\
\hline $\operatorname{Lon}_{2}$ & Lat $_{1}$ & $d_{1,2,1}$ & $\ldots$ & $d_{N_{m a t}, 2,1}$ \\
\hline$\vdots$ & $\vdots$ & $\vdots$ & & $\vdots$ \\
\hline $\operatorname{Lon}_{N_{l o n}}$ & $L_{a} t_{1}$ & $d_{1, N_{l o n}, 1}$ & $\ldots$ & $d_{N_{m a t}, N_{l o n}, 1}$ \\
\hline$\vdots$ & $\vdots$ & $\vdots$ & & \\
\hline $\operatorname{Lon}_{1}$ & $\operatorname{Lat}_{N_{l a t}}$ & $d_{1,1, N_{l a t}}$ & & $d_{N_{m a t}, 1, N_{l a t}}$ \\
\hline $\operatorname{Lon}_{2}$ & $\operatorname{Lat}_{N_{l a t}}$ & $d_{1,2, N_{l a t}}$ & $\ldots$ & $d_{N_{m a t}, 2, N_{l a t}}$ \\
\hline$\vdots$ & . & & & $\vdots$ \\
\hline on $_{N_{l o n}}$ & Lat $_{N_{\text {lat }}}$ & $N_{l o n}, N_{l a}$ & $\ldots$ & $l_{N_{\text {mat }}, N_{\text {lon }}, N}$ \\
\hline
\end{tabular}

It is required that $d_{q, i, j} \leq d_{q+1, i, j}$.

\section{4 sac}

SAC files hold the time history of one component of the solution at a fixed point in space. A detailed description of the SAC format can be found at http: / / www.iris.edu/manuals/sac/manual.html. In the tools directory, we provide a simplified Matlab reader of SAC files called readsac.m. Note that only some of the header information is parsed by this reader:

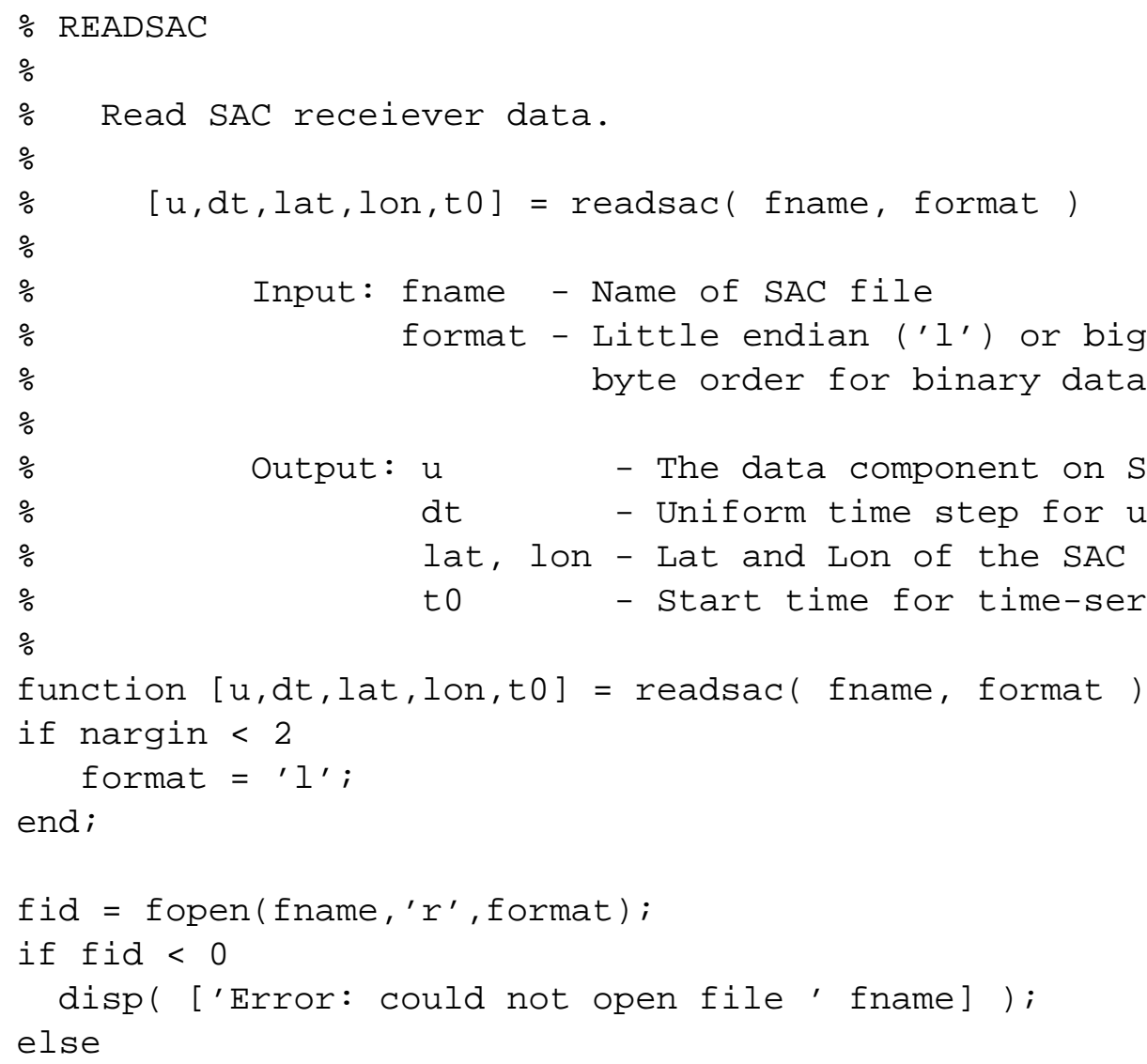




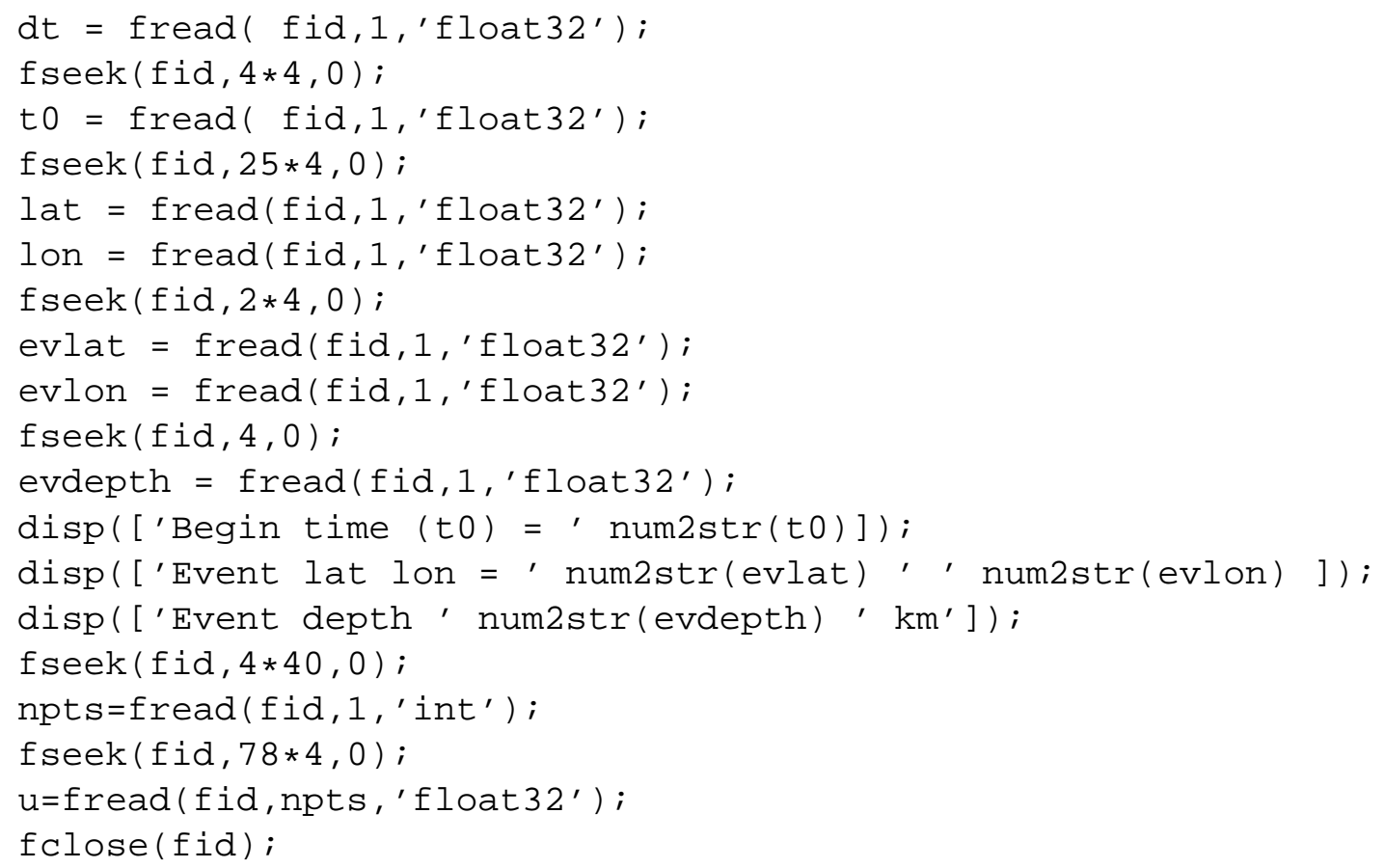

\section{5 image}

Images files hold two-dimensional data on a composite grid and are written in a binary format. The header of the file starts with two integers: the precision ( 4 for single precision, 8 for double precision), and the number of patches. After that follows header info for each patch, consisting of the grid size $h$ (a double precision floating point number) and four integers holding the starting and ending indices for each patch. The header is followed by the two-dimensional data on each patch, consisting of one single or double precision floating point number for each grid point, stored in column-first order.

The exact format follows from the Matlab function tools/readimagepatch.m which is provided in the source distribution of $W P P$ :

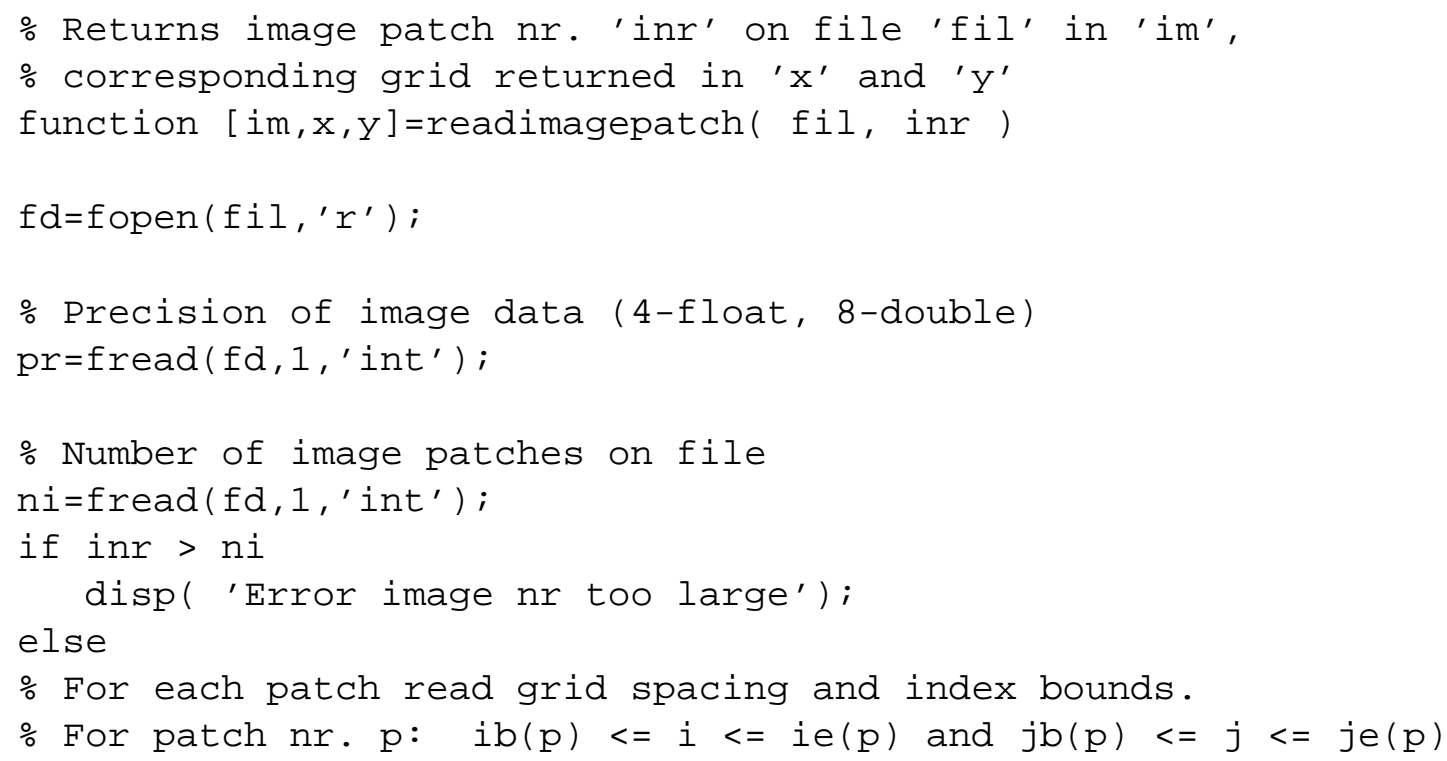




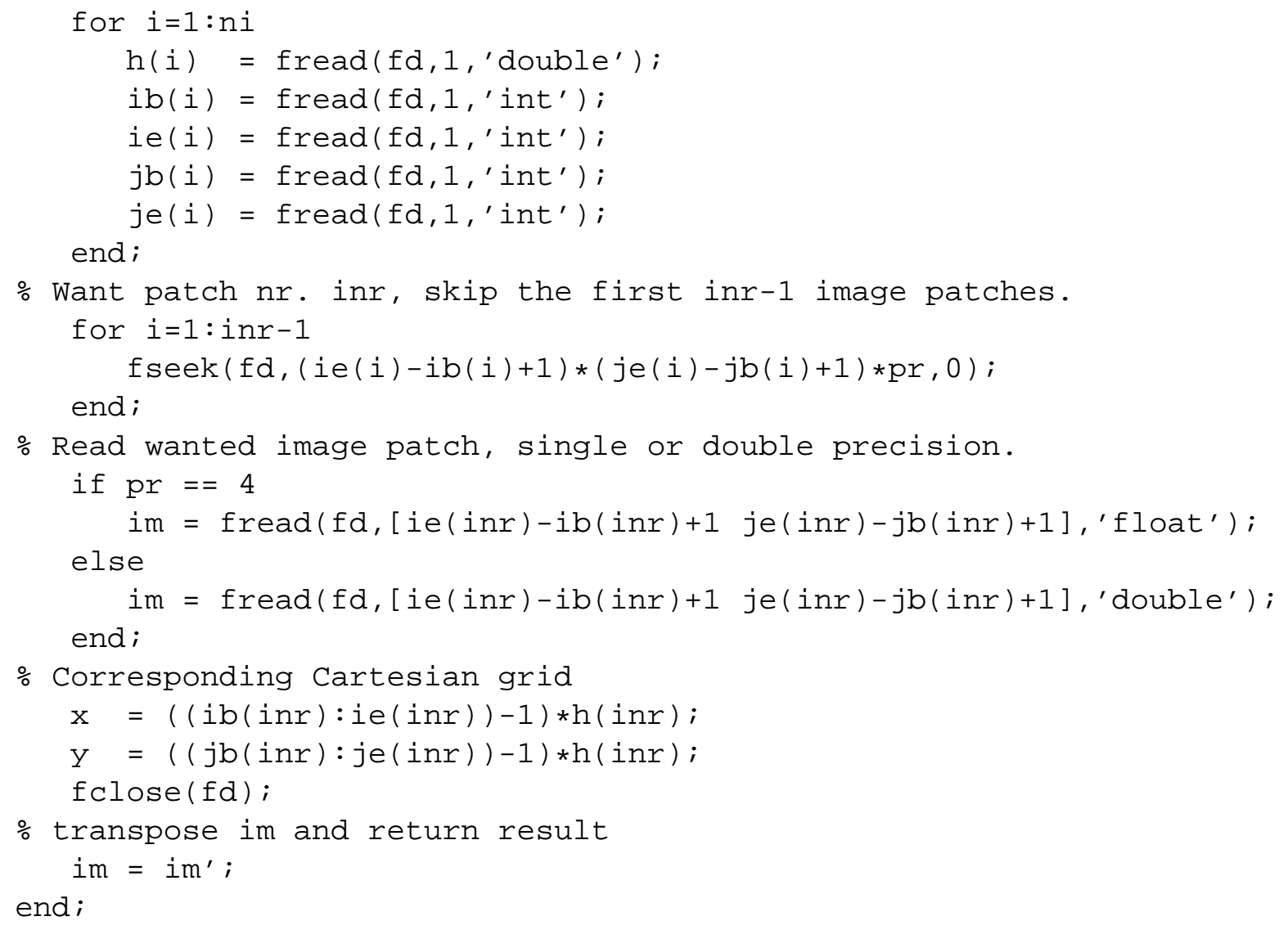

In this implementation, $f d$ is a file descriptor variable. The Matlab functions fopen and $f r e a d$ perform binary I/O similarly to the $\mathrm{C}$ functions with the same names.

Note that the above matlab function reads one image patch from an image file into the Matlab matrix $i m$. The corresponding Cartesian coordinates are returned in the Matlab vectors $\mathrm{x}$ and $\mathrm{y}$. 


\section{Appendix A}

\section{Installing WPP}

The WPP source code is released under the GNU general public license and can be downloaded from:

https://computation.llnl.gov/casc/serpentine/software.html

\section{A.1 Supported platforms}

WPP and its supporting libraries have been built on Intel based desktops and laptops running LINUX and OSX. It has also been built on various supercomputers such as the large Linux clusters at LLNL (currently zeus, hera and atlas), as well as IBM BG/L and BG/P. We have built WPP using Gnu, Intel, or IBM compilers. Our experience is that WPP is likely to build if the underlying third party libraries can be built. Currently, we are using the following compiler versions:

Gnu: $\quad$ t++/gcc/gfortran versions 4.1.2-4.4.2

Intel: icpc/icc/ifort versions 9.1-11.1

IBM: version info currently unavailable

\section{A.2 Build tools, compilers and MPI-library}

WPP is built using a software construction tool called SCons (scons). SCons performs both configuration and compilation/linking through the scons command, and is in that respect different from the more common configure/make process. Before WPP can be built, you must install SCons, which relies on the scripting language Python (v2.3.5 or higher from www . python.org). You must also have access to functioning $\mathrm{C}++$ and Fortran compilers, and a compatible version of the MPI-2 library for parallel communication.

\section{A.2.1 Mac computers running OSX}

We recommend using the MacPorts package manager for installing the required compilers, libraries and scons program. Simply go to www.macports.org, and install macports on your system. With that in place, you can use the port command as follows

shell> port install gcc44

shell> port install mpich2

shell> port install scons

Here, gcc44 refers to version 4.4 of the Gnu compiler suite. Compiler versions are bound to change with time. Before you install gcc, make sure it is compatible with the mpich2 package. 


\section{A.2.2 Linux machines}

Assuming python is already installed, it is straightforward to install scons. First download it from the website www.scons.org (v1.20) and unpack it. To install SCons, simply invoke the following command from the top level directory:

shell> python setup.py install --prefix=/dir/of/your/choice

Note: scons and python can be installed anywhere in your path. Typically people have them installed in /usr/local/bin, but if you don't have root access any other directory reachable by your UNIX path will work.

\section{A.3 Directory structure}

To unpack the WPP source code, you place the downloaded file wpp-version-2.0.tar.gz in the desired location and issue the following commands:

shell> gunzip wpp-version-2.0.tar.gz

shell> tar xvf wpp-version-2.0.tar

Afterwards, you will find a new directory named wpp-version-2.0, which contains several files and subdirectories:

- LICENSE. txt License information.

- INSTALL. txt Information about how to build WPP.

- KNOWN-BUGS . txt List of known problems, porting issues, or bugs.

- README.txt

- TPL. txt Build instructions for third party libraries.

- configs Directory containing scons configuration files.

- $\operatorname{src} \mathrm{C}++$ and Fortran source code of WPP.

- tools Matlab scripts for post processing and analysis.

- examples Sample input files.

- SConstruct A SCons "makefile" (don't change this file!).

- wave.py Python script used to print "WPP Lives" at end of successful builds.

\section{A.4 Compiling and Linking WPP (without the cencalvm library)}

The best way of getting started is to first build WPP without the cencalvm library. This process should be very straight forward and the resulting $W P P$ executable supports all commands except the ef $i$ le command. If you need to use the efile command, it is straight forward to recompile WPP once the cencalvm and supporting libraries have been installed ( $\S$ A.5).

Start by familiarizing yourself with the wpp source code by going to the main wpp directory and listing it 
shell> cd wpp-version-2.0

shell> ls

Go into the configs directory:

shell> cd configs

Use your favorite text editor to inspect the available configuration files and determine which file most closely resembles your system: amac.py is used on an Intel Mac, tux.py is used on a Linux workstation, and atlas.py is used on a large Linux supercomputer. Make a copy of this file (amac.py in this case):

shell> cp amac.py my.py

You need to assign the environment variable WPPCONF IG, which enables WPP to find your configuration file. Add a line in your /. cshrc file (or equivalent):

setenv WPPCONFIG/my/installation/dir/wpp-version-2.0/configs/my.py

Open up a new shell (command window) and verify that WPPCONFIG was assigned properly:

shell> printenv WPPCONFIG

Using your favorite text editor, you need to modify the my - py file to properly reflect the directory structure of your system, the names and locations of your compilers, and the location of the mpich library. You may also need to modify the compiler flags.

shell>cd/my/installation/dir/wpp-version-2.0/configs

shell> emacs my.py

...

Note that you should only uncomment the line which adds -DENABLE_ETREE to CXXFLAGS after the cencalvm library has been installed (i.e., not now).

To compile WPP, you go to the main wpp directory and issue the scons command:

shell>cd/my/installation/dir/wpp-version-2.0

shell> scons

If all goes well, the "WPP lives banner" is shown after the scons command is completed:
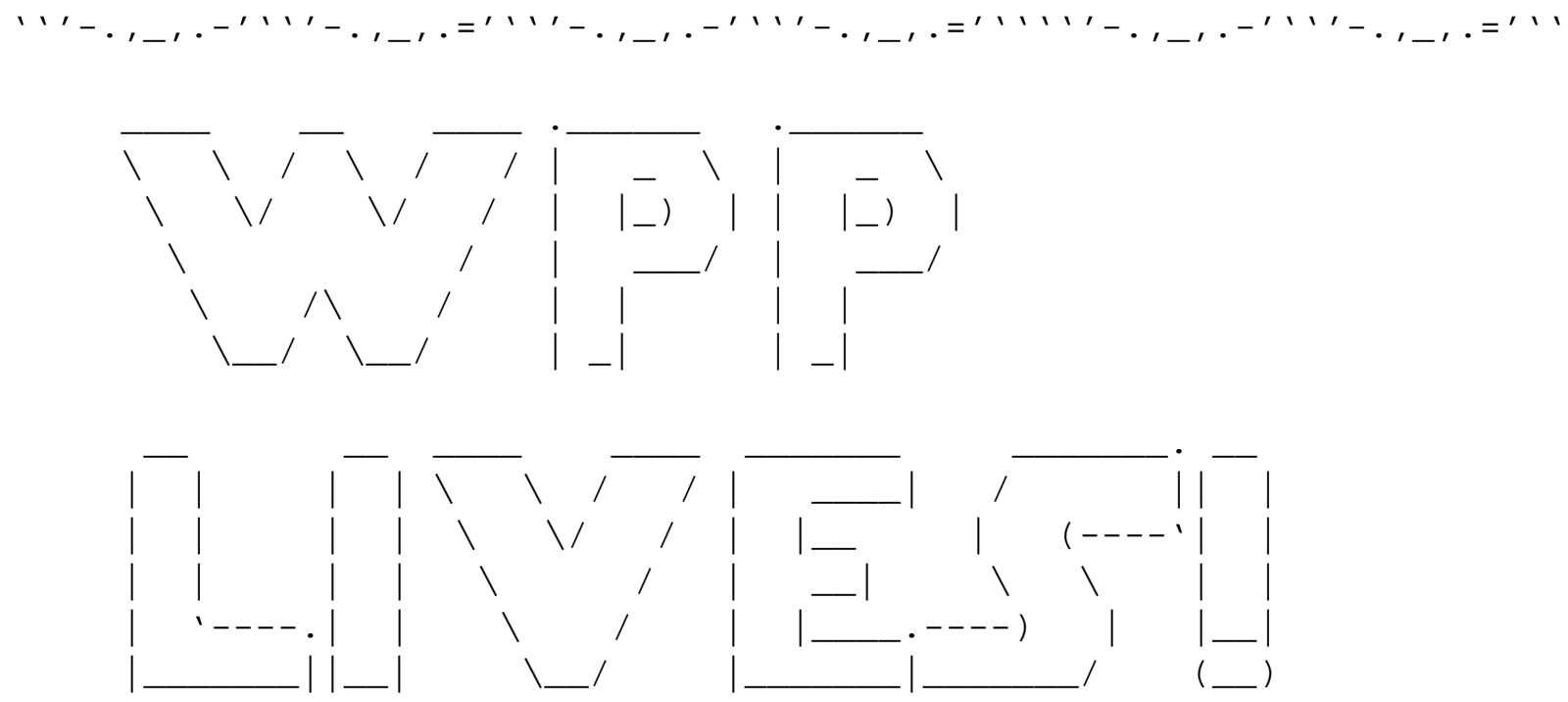
If the scons command results in some error, carefully review these messages. First make sure that scons was able to find your configuration file (my • py). If not, review the above steps for setting the WPPCONFIG environment variable. If you still have problems, make sure that my • py contains correct locations of compilers, libraries, and that appropriate compiler and link flags have been set.

By default, the WPP executable is located in

/my/installation/dir/wpp-version-2.0/optimize_v2.0/wpp

It can be convenient to add this directory to your PATH environment variable (i.e., modify your . cshrc file).

You can also build a debug version of $W P P$ by adding the debug=1 option to scons,

shell>cd/my/installation/dir/wpp-version-2.0

shell> scons debug=1

That executable will be located in

/my/installation/dir/wpp-version-2.0/debug_v2.0/wpp

\section{A.4.1 How does scons work?}

The main input file for scons is

wpp-version-2.0/sconstruct

This file tells scons to initialize some variables and then invokes your setup file

wpp-version-2.0/configs/my ·py

The SConstruct file then finalizes the variables controlling the compiling and linking stages. It is at this point it scans CXXFLAGS for DENABLE_ETREE, and if found adds three extra libraries: cencalvm, etree, and proj. The actual compiling and linking is controlled and preformed by

wpp-version-2.0/src/SConscript

which holds the list of all source code files that are needed to construct the WPP executable. Refer to www.scons.org for a detailed user's guide to the scons build system.

\section{A.5 Installing cencalvm and its supporting libraries}

The cencalvm library was developed by Brad Aagaard at USGS. Both the cencalvm library and the Etree data base files with the material model of Northern California can be downloaded from

WwW.sf06simulation.org/geology/velocitymodel

The installation process which is outlined below is described in detail in the document wWw.sf06simulation.org/geology/velocitymodel/querydoc/INSTALL.html

Note that three libraries need to be installed: euclid (etree), proj4, and cencalvm. In order for WPP to use them, they should all be installed in the same directory and you should assign that directory to the WPP_TPL environment variable. Add a line in your /. cshrc file (or equivalent):

setenv WPP_TPL /my/third/party/directory 
As before, you need to open a new shell or source your .cshrc file before these changes take effect.

Note that the euclid library must be installed manually by explicitly copying all include files to the include directory and all libraries to the lib directory,

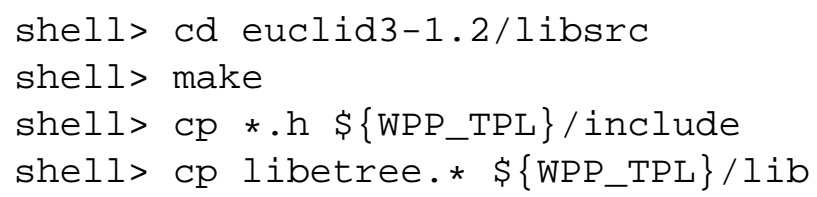

The proj4 library should be configured to be installed in WPP_TPL. This is accomplished by

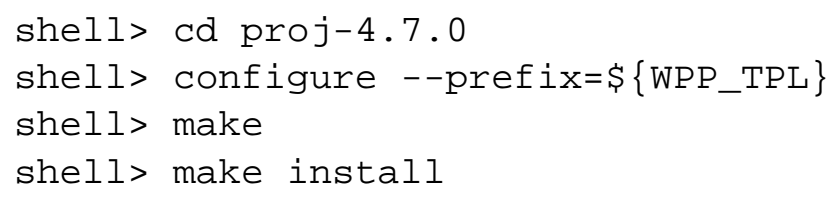

The cencalvm library should also be configured to be installed in WPP_TPL. You also have to help the configure script finding the include and library files for the proj 4 and etree libraries,

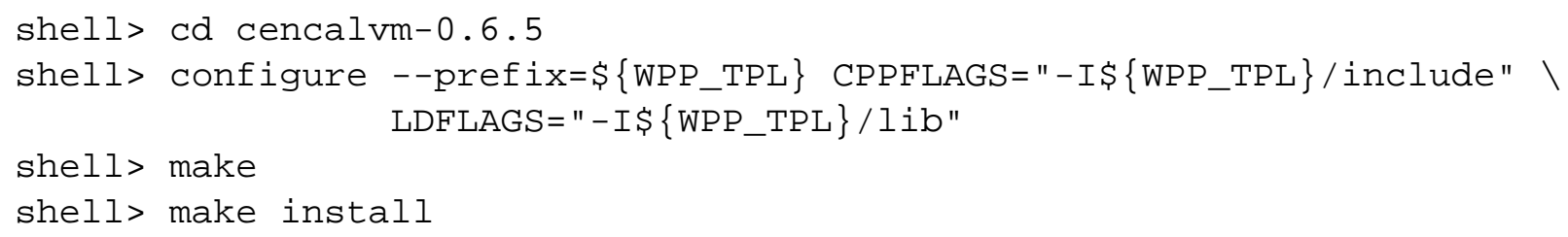

To verify that the libraries have been installed properly, you should go to the WPP_TPL directory and list the lib subdirectory. You should see the following files (on Mac OSX machines, the .so extension is replaced by .dylib ):

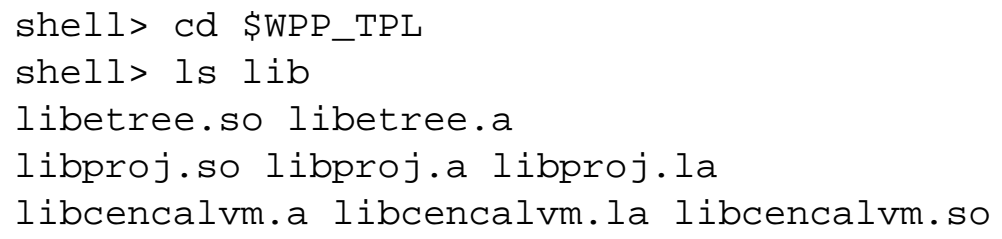

Furthermore, if you list the include subdirectory, you should see include files such as

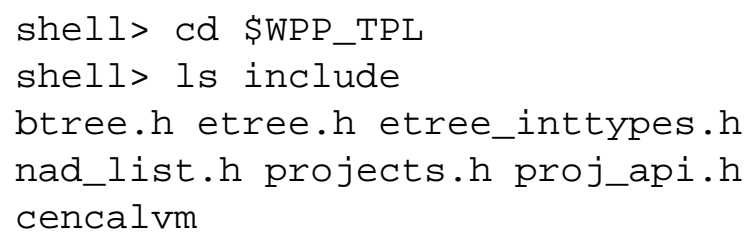

Note that the include files for cencalvm are in a subdirectory with the same name.

Once you have successfully installed all three libraries, it is easy to re-configure WPP to use them. Simply edit the scons configuration file (my • py) to add -DENABLE_ETREE to CXXFLAGS, i.e.,

env.Append (CXXFLAGS = ' -DENABLE_ETREE' $)$

You then need to re-compile WPP. Go to the wpp main directory and re-run scons:

shell>cd/my/installation/dir/wpp-version-2.0

shell> scons

As before, if all goes well, the "WPP lives banner" is shown after the scons command is completed. 


\section{Appendix B}

\section{Testing the WPP installation}

Once $W P P$ has been installed, it is a good idea to verify that the code works properly. For this purpose, we provide test scripts in the examples directory. With each input file xyz. in, there is a corresponding output file named xyz . out. Note that when WPP runs in parallel, some of the output can appear in a different order. The most important aspect of these tests is to verify the reported errors in the numerical solutions, which is reported near the end of the output files. These numbers should be independent of the number of MPI processes on a given machine, but can vary slightly from one type of hardware to another, due to roundoff errors in floating point arithmetic. Note that some of the tests use a significant number of grid points and will only fit in memory on larger machines.

\section{B.1 Method of manufactured solutions}

The method of manufactured solutions (also know as twilight zone testing) provides a general way of testing the accuracy of numerical solutions of partial differential equations, including effects of heterogeneous material properties and various boundary conditions on complex geometries. The test scripts can be found in the directory

\section{.../wpp-version-2.0/examples/twilight}

In the twilight zone testing module of WPP, we take the material properties to be

$$
\begin{aligned}
& \rho(x, y, z)=A_{\rho}\left(2+\sin \left(\omega_{m} x+\theta_{m}\right) \cos \left(\omega_{m} y+\theta_{m}\right) \sin \left(\omega_{m} z+\theta_{m}\right)\right), \\
& \mu(x, y, z)=A_{\mu}\left(3+\cos \left(\omega_{m} x+\theta_{m}\right) \sin \left(\omega_{m} y+\theta_{m}\right) \sin \left(\omega_{m} z+\theta_{m}\right)\right), \\
& \lambda(x, y, z)=A_{\lambda}\left(2+\sin \left(\omega_{m} x+\theta_{m}\right) \sin \left(\omega_{m} y+\theta_{m}\right) \cos \left(\omega_{m} z+\theta_{m}\right)\right) .
\end{aligned}
$$

The internal forcing, boundary forcing and initial conditions are chosen such that the exact (manufactured) solution becomes

$$
\begin{aligned}
u_{e}(x, y, z, t) & =\sin \left(\omega\left(x-c_{e} t\right)\right) \sin (\omega y+\theta) \sin (\omega z+\theta), \\
v_{e}(x, y, z, t) & =\sin (\omega x+\theta) \sin \left(\omega\left(y-c_{e} t\right)\right) \sin (\omega z+\theta), \\
w_{e}(x, y, z, t) & =\sin (\omega x+\theta) \sin (\omega y+\theta) \sin \left(\omega\left(z-c_{e} t\right)\right)
\end{aligned}
$$

The values of the material parameters $\left(\omega_{m}, \theta_{m}, A_{\rho}, A_{\lambda}, A_{\mu}\right)$ and the solution parameters $\left(\omega, \theta, c_{e}\right)$, can be modified in the input script. Since the exact solution is know, it is possible to evaluate the error in the numerical solution. By repeating the same test on several grid sizes, it is possible to establish the convergence order of the numerical method.

The basic twilight tests use a single grid, a flat topography, and cover the computational domain $(x, y, z) \in[0,5]^{3}$. These cases are provided in the three scripts: 


\begin{tabular}{|c|c|c|c|}
\hline$N_{x}$ & $h$ & $\left\|w-w_{e}\right\|_{\infty}$ & ratio \\
\hline 31 & $1.667 \cdot 10^{-1}$ & $1.25 \cdot 10^{-1}$ & - \\
\hline 61 & $8.333 \cdot 10^{-2}$ & $3.28 \cdot 10^{-2}$ & 3.81 \\
\hline 121 & $4.167 \cdot 10^{-2}$ & $8.67 \cdot 10^{-3}$ & 3.78 \\
\hline
\end{tabular}

Table B.1: Twilight test: Max norm errors in the vertical displacement component.

gen-twi-1.in gen-twi-2.in gen-twi-3.in

The numerical solution is simulated up to time $t=4.8$ on a grid with $31^{3}, 61^{3}$, and $121^{3}$ grid points, respectively. The corresponding results are given in the three output files

gen-twi-1.out gen-twi-2.out gen-twi-3.out

The errors in max and $L_{2}$ norm in the numerical solution is reported at the bottom of these files and some of these numers are summarized in Table B.1.

To test mesh refinement on a geometry with flat topography, we provide the scripts

mref-twi-1.in mref-twi-2.in mref-twi-3.in

Again the numerical solution is simulated up to time $t=4.8$ on a grid with $31^{3}, 61^{3}$, and $121^{3}$ grid points, respectively. A refined mesh with half the grid size is used near the free surface, in $0 \leq z \leq 2$. The corresponding results are given in the three output files

mref-twi-1.out mref-twi-2.out mref-twi-3.out

Non-planar free surfaces are tested by the scripts

gauss-twi-1.in gauss-twi-2.in gauss-twi-3.in

In this case, the free surface is a Gaussian hill and the numerical solution is simulated up to time $t=0.8$ on a grid with $31^{3}, 61^{3}$, and $121^{3}$ grid points, respectively. The curvilinear grid covers the domain between the free surface and $z=0.25$, and a single Cartesian grid covers the remainder of the computational domain $(0.25 \leq z \leq 1)$. The corresponding results are given in the three output files

gauss-twi-1.out gauss-twi-2.out gauss-twi-3.out

Note that some image files are generated by these scripts and placed in the sub-directories gauss_31, gauss_61, and gauss_121, respectively. We encourage the user to look at these image files, for example by reading them into matlab/octave using the script tools/readimagepatch.m.

\section{B.2 Lamb's problem}

The WPP installation can be tested further by solving Lamb's problem, i.e., the motion due to a vertical point force applied on the free surface. Here, we only have access to the vertical component of the exact solution along the free surface, when the source time function is of the type "VerySmoothBump" with freq=1. This problem tests the implementation of a point force and (to some extent) the supergrid far field boundary condition. The input files can be found in the directory 
shell>cd wpp-version-2.0/examples/lambtest

shell> ls

Lambtest1.in Lambtest2.in Lambtest3.in Lambtest4.in

Here we provide input files with four different grid sizes, with $116^{2} \times 59,231^{2} \times 116,461^{2} \times 231$, and $921^{2} \times 461$ grid points, respectively. Be aware that the finest grid uses about 391 Million grid points and can only be run on a sufficiently large machine. The corresponding output files are given in

Lambtest1.out Lambtest2.out Lambtest3.out Lambtest4.out

The most important information is near the end of these files, where the error in the numerical solution is reported. You migth also find it interesting to compare your execution times with the ones we got. 


\section{Index}

block parameters

vp, vs, rho, vpgrad, vsgrad, rhograd, x1, x2, y1, $\mathrm{y} 2, \mathrm{z} 1, \mathrm{z} 2,55$

boundary_conditions parameters

lx, hx, ly, hy, lz, hz, 66

boundary_conditions values

clayton-engquist, energy-absorbing, stress-free,

dirichlet, neumann, supergrid, 66

command

block, 54

boundary_conditions, 66

developer, 66

efile, 55

fileio, 50

globalmaterial, 57

gmt, 62

grid, 51

ifile, 56

image, 60

material, 57

pfile, 56

prefilter, 54

refinement, 59

sac, 59

source, 52

supergrid, 65

testlamb, 63

testpointsource, 64

time, 52

topography, 58

twilight, 63

command line options

-v version info, 7

coordinate system, 8

developer parameters

cfl_number output_load, output_timing, interpo-

lation, ctol, cmaxit, log_energy, print_energy, mpiio, iotiming, 67 efile parameters

logfile, vsmin, vpmin, query, resolution, access, etree, xetree, 56

examples, 39

earthquake, 46

grenoble, 43

lambs, 39

scec, 40

fileformats, 68

ifile, 69

image, 71

pfile, 69

sac, 70

topography, 68

fileio parameters

path, verbose, printcycle, pfs, nwriters, 51

geographic coordinates, 9

globalmaterial parameters

vpmin, vsmin, 57

gmt parameters

file, 62

grid parameters

location - az, lat, lon, 52

size - $x, y, z, h, n x, n y, n z, 52$

grid size, 18

gridsize, 11

ifile parameters

filename, 57

image mode options

ux, uy, uz, rho, lambda, mu, p, s, div, curl, veldiv, velcurl, velmag, lat, lon, hvelmax, vvelmax, topo, grid, uxerr, uyerr, uzerr, fx, fy, $\mathrm{fz}, 62$

image parameters

file, mode, precision, 61

location - $\mathrm{x}, \mathrm{y}, \mathrm{z}, 61$

timing - time, timeInterval, cycle, cycleInterval, 61 
installation, 73

basic, 74

cencalvm, 76

directories, 74

efile, 76

platforms, 73

tools, 73

material, 22

material parameters

id, vp, vs, rho, vpgrad, vsgrad, rhograd, vp2, vs2, rho2, 57

mesh refinement, 31

output options, 34

parallel execution, 6

pfile parameters

filename, directory, smoothingsize, vpmin, vs$\mathrm{min}$, rhomin, flatten, 56

prefilter parameters

fc, maxfreq, 54

refinement parameters

zmax, 59

sac parameters

location - x, y, z, lat, lon, depth, topodepth, 60

sta, file, type, writeEvery, eventDate, eventTime, nsew, velocity, usgsformat, sacformat, 60

source parameters

Aki and Richards - strike, dip, rake, 54

location - x, y, z, depth, topodepth, lat, lon, 54

moment - m0, mxx, myy, mzz, mxy, mxz, myz, 54

point force - f0, fx, fy, fz, 54

$\mathrm{t} 0$, freq, type, 54

source time dependence

GaussianInt, Gaussian, RickerInt, Ricker, Ramp, Triangle, Sawtooth, Smoothwave, VerySmoothBump, Brune, BruneSmoothed, Liu, 54

sources, 11

srun, 6

supergrid parameters

thickness, damping, 66

testing, 78

lambs, 79 twilight, 78

testlamb parameters

$\mathrm{x}, \mathrm{y}, \mathrm{cp}, \mathrm{rho}, \mathrm{fz}, 64$

testpointsource parameters

$\mathrm{x}, \mathrm{y}, \mathrm{z}, \mathrm{cp}, \mathrm{cs}, \mathrm{rho}, \mathrm{m} 0, \mathrm{mxx}, \mathrm{mxy}, \mathrm{mxz}, \mathrm{myy}$, myz, mzz, f0, fx, fy, fz, freq, t0, type, 65

time parameters

t, steps, 52

topography, 28

topography parameters

gaussianAmp, gaussianXc, gaussian Yc, gaussianLx, gaussianLy, 58

input, file, resolution, zmax, order, smooth, 58

twilight parameters

errorlog, omega, c, phase, momega, mphase, amprho, ampmu, amplambda, 63

units, 8 


\section{Bibliography}

[1] K. Aki and P.G. Richards. Quantitative Seismology. University Science Books, second edition, 2002.

[2] D. Appelö and N. A. Petersson. A stable finite difference method for the elastic wave equation on complex geometries with free surfaces. Comm. Comput. Phys., 5:84-107, 2009.

[3] S. M. Day et al. Test of 3D elastodynamic codes: Final report for lifelines project 1A01. Technical report, Pacific Earthquake Engineering Center, 2001.

[4] P. Goldstein, D. Dodge, M. Firpo, and L. Miner. International Handbook of Earthquake and Engineering Seismology, volume 81B, chapter SAC2000: Signal processing and analysis tools for seismologists and engineers, pages 1613-1614. International Association of Seismology and Physics of the Earth's Interior, 2003.

[5] B. Gustafsson, H.-O. Kreiss, and J. Oliger. Time dependent problems and difference methods. WileyInterscience, 1995.

[6] H. Lamb. On the propagation of tremors over the surface of an elastic solid. Phil. Trans. Roy. Soc. London, Ser. A, 1904.

[7] P. Liu, R. J. Archuleta, and S. H. Hartzell. Perdiction of broadband ground-motion time histories: Hybrid low/high-frequency method with correlated random source parameters. Bulletin of the Seismological Society of America, 96:2118-2130, 2006.

[8] H. M. Mooney. Some numerical solutions for Lamb's problem. Bulletin of the Seismological Society of America, 64, 1974.

[9] S. Nilsson, N.A. Petersson, B. Sjögreen, and H.-O. Kreiss. Stable difference approximations for the elastic wave equation in second order formulation. SIAM J. Numer. Anal., 45:1902-1936, 2007.

[10] N. A. Petersson and B. Sjogreen. Stable grid refinement and singular source discretization for seismic wave simulations. LLNL-JRNL 419382, Lawrence Livermore National Laboratory, 2009. submitted to Comm. Comput. Phys.

[11] P. Wessel and W. H. F. Smith. New, improved version of generic mapping tools released. In EOS trans. $A G U$, volume 79, page 579, 1998. 